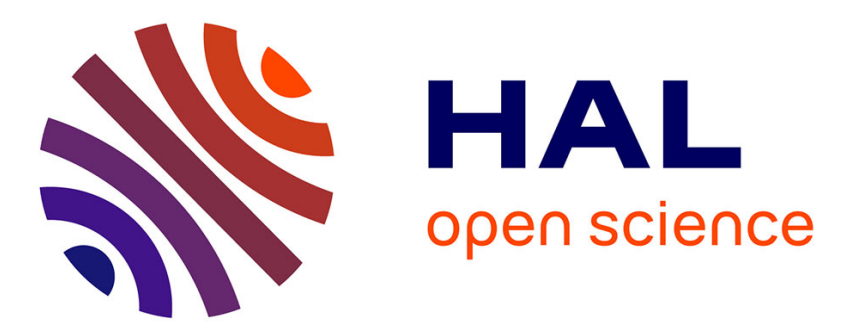

\title{
Convergence analysis of a mixed finite volume scheme for an elliptic-parabolic system modeling miscible fluid flows in porous media
}

Claire Chainais-Hillairet, Jérôme Droniou

\section{- To cite this version:}

Claire Chainais-Hillairet, Jérôme Droniou. Convergence analysis of a mixed finite volume scheme for an elliptic-parabolic system modeling miscible fluid flows in porous media. SIAM Journal on Numerical Analysis, 2007, 45 (5), pp.2228-2258. 10.1137/060657236 . hal-00022910

\section{HAL Id: hal-00022910 https://hal.science/hal-00022910}

Submitted on 14 Apr 2006

HAL is a multi-disciplinary open access archive for the deposit and dissemination of scientific research documents, whether they are published or not. The documents may come from teaching and research institutions in France or abroad, or from public or private research centers.
L'archive ouverte pluridisciplinaire HAL, est destinée au dépôt et à la diffusion de documents scientifiques de niveau recherche, publiés ou non, émanant des établissements d'enseignement et de recherche français ou étrangers, des laboratoires publics ou privés. 


\title{
Convergence analysis of a mixed finite volume scheme for an elliptic-parabolic system modeling miscible fluid flows in porous media
}

\author{
Claire Chainais-Hillairet ${ }^{1}$, Jérôme Droniou ${ }^{2}$. \\ $11 / 04 / 2006$
}

\begin{abstract}
We study a Finite Volume discretization of a strongly coupled elliptic-parabolic PDE system describing miscible displacement in a porous medium. We discretize each equation by a finite volume scheme whose properties are to handle a wide variety of unstructured grids (in any space dimension) and to give strong enough convergence to handle the nonlinear coupling of the equations. We prove the convergence of the scheme as the time and space steps go to 0 . Finally, we provide numerical results which show that, from a practical point of view, the scheme behaves very well.
\end{abstract}

Mathematics Subject Classification: 65M12, 65M30, 65N12, 65N30, 76S05, 76R99.

Keywords: finite volume methods, porous medium, miscible fluid flow, convergence analysis, numerical tests.

\section{Introduction}

\subsection{Miscible displacement in porous media}

The mathematical model for the single-phase miscible displacement of one fluid by another in a porous medium, in the case where the fluids are considered incompressible, is an elliptic-parabolic coupled system $[2,4]$. Let $\Omega$ be a bounded domain of $\mathbb{R}^{d}(d=2$ or 3$)$ representing the reservoir and $] 0, T[$ be the time interval. The unknowns of the problem are $p$ the pressure in the mixture, $\mathbf{U}$ its Darcy velocity and $c$ the concentration of the invading fluid.

We denote by $\Phi(x)$ and $\mathbf{K}(x)$ the porosity and the absolute permeability tensor of the porous medium, $\mu(c)$ the viscosity of the fluid mixture, $\widehat{c}$ the injected concentration, $q^{+}$and $q^{-}$the injection and the production source terms. If we neglect gravity, the model writes:

$$
\begin{aligned}
& \begin{cases}\operatorname{div}(\mathbf{U})=q^{+}-q^{-} & \text {in }] 0, T[\times \Omega, \\
\mathbf{U}=-\frac{\mathbf{K}(x)}{\mu(c)} \nabla p & \text { in }] 0, T[\times \Omega,\end{cases} \\
& \left.\Phi(x) \partial_{t} c-\operatorname{div}(D(x, \mathbf{U}) \nabla c-c \mathbf{U})+q^{-} c=q^{+} \widehat{c} \quad \text { in }\right] 0, T[\times \Omega
\end{aligned}
$$

where $D$ is the diffusion-dispersion tensor including molecular diffusion and mechanical dispersion. It is given by

$$
D(x, \mathbf{U})=\Phi(x)\left(d_{m} \mathbf{I}+|\mathbf{U}|\left(d_{l} E(\mathbf{U})+d_{t}(\mathbf{I}-E(\mathbf{U}))\right)\right),
$$

where $\mathbf{I}$ is the identity matrix, $d_{m}$ is the molecular diffusion, $d_{l}$ and $d_{t}$ are the longitudinal and transverse dispersion coefficients and $E(\mathbf{U})=\left(\frac{\mathbf{U}_{i} \mathbf{U}_{j}}{|\mathbf{U}|^{2}}\right)_{1 \leq i, j \leq d}$. Laboratory experiments have found that the longitudinal dispersivity $d_{l}$ is much greater than the transverse dispersivity $d_{t}$ and that the diffusion coefficient is very small by comparison.

\footnotetext{
${ }^{1}$ Laboratoire de Mathématiques, UMR CNRS 6620, Université Blaise Pascal, 63177 Aubière Cedex, France. email: Claire.Chainais@math.univ-bpclermont.fr

${ }^{2}$ Département de Mathématiques, UMR CNRS 5149, CC 051, Université Montpellier II, Place Eugène Bataillon, 34095 Montpellier cedex 5, France. email: droniou@math.univ-montp2.fr
} 
In reservoir simulation, the boundary $\partial \Omega$ is typically impermeable. Therefore, if $\mathbf{n}$ denotes the exterior normal to $\partial \Omega$, the system (1.1) — (1.2) is supplemented with no flow boundary conditions:

$$
\begin{cases}\mathbf{U} \cdot \mathbf{n}=0 & \text { on }] 0, T[\times \partial \Omega, \\ D(x, \mathbf{U}) \nabla c \cdot \mathbf{n}=0 & \text { on }] 0, T[\times \partial \Omega .\end{cases}
$$

An initial condition is also added:

$$
c(x, 0)=c_{0}(x)
$$

Because of the homogeneous Neumann boundary conditions on $\mathbf{U}$, the injection and production source terms have to satisfy the compatibility condition $\int_{\Omega} q^{+}(., x) d x=\int_{\Omega} q^{-}(., x) d x$ in $] 0, T[$, and since the pressure is defined only up to an arbitrary constant, we normalize $p$ by the following condition:

$$
\left.\int_{\Omega} p(., x) d x=0 \text { on }\right] 0, T[
$$

The viscosity $\mu$ is usually determined by the following mixing rule:

$$
\mu(c)=\mu(0)\left(1+\left(M^{1 / 4}-1\right) c\right)^{-4} \text { on }[0,1]
$$

where $M=\frac{\mu(0)}{\mu(1)}$ is the mobility ratio (we extend $\mu$ to $\mathbb{R}$ by letting $\mu=\mu(0)$ on $]-\infty, 0[$ and $\mu=\mu(1)$ on ] $1, \infty[)$. The porosity $\Phi$ and the permeability $\mathbf{K}$ are in general assumed to be bounded from above and from below by positive constants (or positive multiples of $\mathbf{I}$ for the tensor $\mathbf{K}$ ).

In [15], Feng proved the existence of a weak solution to the problem (1.1)-(1.6), in the two-dimensional case and with $d_{l} \geq d_{t}>0$ and $d_{m}>0$. This result has been generalized by Chen and Ewing in [3] to the three-dimensional case and with gravity effects and various boundary conditions. At high flow velocities the effects of mechanical dispersion are much greater than those of molecular diffusion. Therefore, Amirat and Ziani studied in [1] the asymptotic behaviour of the weak solution as $d_{m}$ goes to 0 and they proved existence of a weak solution in the case where $d_{m}=0$.

From a numerical point of view, different kind of methods have already been developed for this problem. The key point is that the equation (1.2) on $c$ is a convection-dominated equation, which is not well adapted to the discretization by finite difference or finite element methods. Different ways of discretization of this equation were studied; in general the pressure equation is discretized by a finite element method. Douglas, Ewing and Wheeler [6] used a mixed finite element method for the pressure equation and a Galerkin finite element method for the concentration equation. In [19], Russell introduced a modified method of characteristic for the resolution of (1.2), while (1.1) is solved by a finite element method. Then, Ewing, Russell and Wheeler [10] combined mixed finite element method for (1.1) and modified method of characteristic for (1.2). In [20,21], the authors still used a mixed finite element method for (1.1) but developed a Euler Lagrangian Localized Adjoint Method for (1.2).

Convergence of numerical schemes to (1.1) - (1.6) (or connected problems) has already been studied (see $[5],[6],[11],[12],[17] \ldots)$. But, to our best knowledge, these proofs of convergence are based on a priori error estimates, which need regularity assumptions on the solution $(p, \mathbf{U}, c)$ to the continuous problem. Such regularity does not seem provable in general, all the more if we take a discontinuous permeability tensor (which is expected in field applications, see [20]).

Finite volume methods are well adapted to the discretization of conservation laws: see for instance the book by Eymard, Gallouët, Herbin, [13]. They provide numerical schemes as well for elliptic equations as for convection-dominated parabolic equations. But, because of the anisotropic diffusion in (1.1) (due to $\mathbf{K}(x))$ and of the dispersion terms in (1.2) - (1.3), the standard four point finite volume schemes cannot be used here. Besides, as said above, (1.2) is convection-dominated and, therefore, a good approximation of $\mathbf{U}$ must be used in the discretization of (1.2) if one wants to obtain admissible numerical results. In [9], Droniou and Eymard recently proposed a mixed finite volume scheme which handles anisotropic heterogeneous diffusion problems on any grid and precisely provides, for equations such as (1.1), good 
approximations of $\mathbf{U}$; this scheme is therefore a natural candidate to discretize such coupled problems as (1.1) - (1.6), all the more as it has been shown to behave well from a numerical point of view.

In this paper, we extend the mixed finite volume scheme of [9] to a system, presented in Section 1.2, which generalizes (1.1)-(1.6). Section 2 is devoted to the definition of the scheme and the statement of the main results: existence and uniqueness of an approximate solution and its convergence to the solution of the continuous problem as the time and space steps tend to 0 . A priori estimates on the approximate solution are established in Section 3 and, in Section 4, we prove the existence and uniqueness of the solution to our scheme and present an efficient method to compute it. The proof of convergence of the scheme is made in Section 5, under no regularity assumption on the solution to the continuous problem. Section 6 is devoted to numerical experiments, which show that the scheme behaves very well in practical situations. Section 7 is an appendix containing a few technical results.

\subsection{Formulation of the problem and assumptions}

Let us now rewrite the problem (1.1) - (1.6) under the following synthetized and more general form (notice that, from now on, we use a bar to denote the exact solutions, and we keep the letters without bar to denote approximate solutions):

$$
\begin{aligned}
& \begin{cases}\operatorname{div}(\overline{\mathbf{U}})=q^{+}-q^{-} & \text {in }] 0, T[\times \Omega, \\
\overline{\mathbf{U}}=-A(\cdot, \bar{c}) \nabla \bar{p} & \text { in }] 0, T[\times \Omega, \\
\int_{\Omega} \bar{p}(\cdot, x) d x=0 & \text { on }] 0, T[, \\
\overline{\mathbf{U}} \cdot \mathbf{n}=0 & \text { on }] 0, T[\times \partial \Omega,\end{cases} \\
& \begin{cases}\Phi \partial_{t} \bar{c}-\operatorname{div}(D(\cdot, \overline{\mathbf{U}}) \nabla \bar{c})+\operatorname{div}(\bar{c} \overline{\mathbf{U}})+q^{-} \bar{c}=q^{+} \widehat{c} & \text { in }] 0, T[\times \Omega, \\
\bar{c}(0, \cdot)=c_{0} & \text { on } \Omega, \\
D(\cdot, \overline{\mathbf{U}}) \nabla \bar{c} \cdot \mathbf{n}=0 & \text { on }] 0, T[\times \partial \Omega .\end{cases}
\end{aligned}
$$

In the sequel, we assume that $\Omega$ is a convex polygonal bounded open subset of $\mathbb{R}^{d}$ and that $T>0$. The assumptions on the data are the following:

$$
\begin{aligned}
& \left(q^{+}, q^{-}\right) \in L^{\infty}\left(0, T ; L^{2}(\Omega)\right) \text { are nonnegative functions s.t. } \\
& \left.\int_{\Omega} q^{+}(\cdot, x) d x=\int_{\Omega} q^{-}(\cdot, x) d x \text { a.e. on }\right] 0, T[, \\
& \exists \alpha_{A}>0 \text { s.t. } A(x, s) \xi \cdot \xi \geq \alpha_{A}|\xi|^{2} \text { for a.e. } x \in \Omega \text {, all } s \in \mathbb{R} \text { and all } \xi \in \mathbb{R}^{d} \text {, } \\
& \exists \Lambda_{A}>0 \text { s.t. }|A(x, s)| \leq \Lambda_{A} \text { for a.e. } x \in \Omega \text { and all } s \in \mathbb{R}, \\
& \left.\widehat{c} \in L^{\infty}(] 0, T[\times \Omega) \text { satisfies: } 0 \leq \widehat{c} \leq 1 \text { a.e. in }\right] 0, T[\times \Omega \text {, } \\
& c_{0} \in L^{\infty}(\Omega) \text { satisfies: } 0 \leq c_{0} \leq 1 \text { a.e. in } \Omega \text {. }
\end{aligned}
$$

Remark 1.1 Since $E(\mathbf{U})=\left(\mathbf{U}_{i} \mathbf{U}_{j} /|\mathbf{U}|^{2}\right)_{1 \leq i, j \leq d}$ is the orthogonal projector on $\mathbb{R} \mathbf{U}$, it is clear that the preceding assumptions are satisfied by the model presented in Section 1.1, provided that $d_{m}>0, d_{l}>0$ and $d_{t}>0$. 
As $\Phi$ does not depend on $t$, the following definition (similar to the one in [15]) of weak solution to (1.7)-(1.8) makes sense.

Definition 1.1 Under assumptions (1.9) - (1.14), a weak solution to (1.7) - (1.8) is $(\bar{p}, \overline{\mathbf{U}}, \bar{c})$ such that

$$
\begin{gathered}
\bar{p} \in L^{\infty}\left(0, T ; H^{1}(\Omega)\right), \quad \overline{\mathbf{U}} \in L^{\infty}\left(0, T ; L^{2}(\Omega)\right)^{d}, \quad \bar{c} \in L^{\infty}\left(0, T ; L^{2}(\Omega)\right) \cap L^{2}\left(0, T ; H^{1}(\Omega)\right), \\
\left.\int_{\Omega} \bar{p}(t, \cdot)=0 \text { for a.e. } t \in\right] 0, T[, \quad \overline{\mathbf{U}}=-A(\cdot, \bar{c}) \nabla \bar{p} \text { a.e. on }] 0, T[\times \Omega, \\
\forall \varphi \in C^{\infty}([0, T] \times \bar{\Omega}), \quad-\int_{0}^{T} \int_{\Omega} \overline{\mathbf{U}} \cdot \nabla \varphi=\int_{0}^{T} \int_{\Omega}\left(q^{+}-q^{-}\right) \varphi, \\
\forall \varphi \in C_{c}^{\infty}\left(\left[0, T[\times \bar{\Omega}), \quad-\int_{0}^{T} \int_{\Omega} \Phi \bar{c} \partial_{t} \varphi+\int_{0}^{T} \int_{\Omega} D(\cdot, \overline{\mathbf{U}}) \nabla \bar{c} \cdot \nabla \varphi-\int_{0}^{T} \int_{\Omega} \bar{c} \overline{\mathbf{U}} \cdot \nabla \varphi+\int_{0}^{T} \int_{\Omega} q^{-} \bar{c} \varphi\right.\right. \\
-\int_{\Omega} \Phi c_{0} \varphi(0, \cdot)=\int_{0}^{T} \int_{\Omega} q^{+} \widehat{c} \varphi .
\end{gathered}
$$

\section{Scheme and main results}

Let us first define the notion of admissible discretization of $\Omega$, and some notations associated with.

Definition 2.1 Let $\Omega$ be a convex polygonal bounded open subset of $\mathbb{R}^{d}$. An admissible discretization of $\Omega$ is given by $\mathcal{D}=(\mathcal{M}, \mathcal{E})$, where:

- $\mathcal{M}$ is a finite family of non empty convex polygonal open disjoint subsets of $\Omega$ (the "control volumes") such that $\bar{\Omega}=\cup_{K \in \mathcal{M}} \bar{K}$.

- $\mathcal{E}$ is a finite family of disjoint subsets of $\bar{\Omega}$ (the "edges" of the mesh), such that, for all $\sigma \in \mathcal{E}$, there exists an affine hyperplane $E$ of $\mathbb{R}^{d}$ and $K \in \mathcal{M}$ verifying: $\sigma \subset \partial K \cap E$ and $\sigma$ is a non empty open convex subset of $E$. We assume that, for all $K \in \mathcal{M}$, there exists a subset $\mathcal{E}_{K}$ of $\mathcal{E}$ such that $\partial K=\cup_{\sigma \in \mathcal{E}_{K}} \bar{\sigma}$. We also assume that, for all $\sigma \in \mathcal{E}$, either $\sigma \subset \partial \Omega$ or $\bar{\sigma}=\bar{K} \cap \bar{L}$ for some $(K, L) \in \mathcal{M} \times \mathcal{M}$.

The $d$-dimensional measure of a control volume $K$ is written $\mathrm{m}(K)$, and the $(d-1)$-dimensional measure of an edge $\sigma$ is $\mathrm{m}(\sigma)$; in the integral signs, $\gamma$ denotes the measure on the edges. If $\sigma \in \mathcal{E}_{K}$, then $\mathbf{n}_{K, \sigma}$ is the unit normal to $\sigma$ outward to $K$. In the case where $\sigma \in \mathcal{E}$ is such that $\bar{\sigma}=\bar{K} \cap \bar{L}$ for $(K, L) \in \mathcal{M} \times \mathcal{M}$, we denote $\sigma=K \mid L$ ( $K$ and $L$ are then called "neighboring control volumes"). We define the set of interior (resp. boundary) edges as $\mathcal{E}_{\text {int }}=\{\sigma \in \mathcal{E} ; \sigma \not \subset \partial \Omega\}$ (resp. $\mathcal{E}_{\text {ext }}=\{\sigma \in \mathcal{E} ; \sigma \subset \partial \Omega\}$ ). For all $K \in \mathcal{M}$ and all $\sigma \in \mathcal{E}, \mathbf{x}_{K}$ and $\mathbf{x}_{\sigma}$ are the respective barycenters of $K$ and $\sigma$.

The size of a discretization $\mathcal{D}$ is $\operatorname{size}(\mathcal{D})=\sup _{K \in \mathcal{M}} \operatorname{diam}(K)$. To study the convergence of our scheme as the size tends to 0 , we need that the following quantity (measuring the regularity of the discretization) stays bounded:

$$
\operatorname{regul}(\mathcal{D})=\sup \left\{\max \left(\frac{\operatorname{diam}(K)^{d}}{\rho_{K}^{d}}, \operatorname{Card}\left(\mathcal{E}_{K}\right)\right) ; K \in \mathcal{M}\right\}
$$

where, for $K \in \mathcal{M}, \rho_{K}$ is the supremum of the radius of the balls contained in $K$. One notices that, with such a definition of $\operatorname{regul}(\mathcal{D})$, we have, for all $K \in \mathcal{M}$,

$$
\operatorname{diam}(K)^{d} \leq \operatorname{regul}(\mathcal{D}) \rho_{K}^{d} \leq \frac{\operatorname{regul}(\mathcal{D})}{\omega_{d}} \mathrm{~m}(K),
$$

where $\omega_{d}$ is the volume of the unit ball in $\mathbb{R}^{d}$. 
Remark 2.1 We ask for very few geometrical properties on the discretization of $\Omega$. This is particularly important since, in real world problems, meshes used in basin and reservoir simulations can be quite irregular and not admissible in the usual finite element or finite volume senses (see [14]).

Our scheme is based on the mixed finite volume scheme introduced in [9] or [8], whose main interests are to handle a wide variety of grids for heterogeneous and anisotropic operators and to nevertheless give strong convergence of approximate gradients. Moreover, this scheme is, as it is natural for finite volume schemes, "fluxes-oriented" and therefore provides natural fluxes for the approximation of $\overline{\mathbf{U}}$, which can then be used in the discretization of the convective term $\operatorname{div}(\bar{c} \overline{\mathbf{U}})$ in the parabolic equation.

The idea is to consider, besides unknowns which approximate the functions $(\bar{p}, \bar{c})$, unknowns which approximate the gradients of these functions, as well as unknowns which stand for the fluxes associated with the differential operators. Thus, if $\mathcal{D}$ is an admissible discretisation of $\Omega$ and $k>0$ is a time step (we always choose time steps such that $N_{k}=T / k$ is an integer), we consider, for all $n=1, \ldots, N_{k}$ and all $K \in \mathcal{M}$, unknowns $\left(p_{K}^{n}, \mathbf{v}_{K}^{n}\right)$ which stand for approximate values of $(\bar{p}, \nabla \bar{p})$ on $[(n-1) k, n k[\times K$ and numbers $F_{K, \sigma}^{n}$ (for $\left.\sigma \in \mathcal{E}_{K}\right)$ which stand for approximate values of $-\int_{\sigma} \overline{\mathbf{U}} \cdot \mathbf{n}_{K, \sigma} d \gamma$ on $[(n-1) k, n k[$. Similarly, the unknowns $\left(c_{K}^{n}, \mathbf{w}_{K}^{n}\right)$ approximate $(\bar{c}, \nabla \bar{c})$ on $\left[(n-1) k, n k\left[\times K\right.\right.$ and the numbers $G_{K, \sigma}^{n}$ (for $\left.\sigma \in \mathcal{E}_{K}\right)$ approximate $\int_{\sigma} D(\cdot, \overline{\mathbf{U}}) \nabla \bar{c} \cdot \mathbf{n}_{K, \sigma} d \gamma$ on $[(n-1) k, n k[$.

The quantities $q_{K}^{+, n}, q_{K}^{-, n}$ and $\widehat{c}_{K}^{n}$ denote the mean values of $q^{+}, q^{-}$and $\widehat{c}$ on $\left[(n-1) k, n k\left[\times K\right.\right.$ and $\Phi_{K}$, $c_{K}^{0}, A_{K}(s)$ and $D_{K}(\xi)$ are the mean values of $\Phi, c_{0}, A(\cdot, s)$ and $D(\cdot, \xi)$ on $K$. We also take positive numbers $\left(\nu_{K}\right)_{K \in \mathcal{M}}$. The equation (1.7) is then discretized by the following way:

$$
\begin{gathered}
\mathbf{v}_{K}^{n} \cdot\left(\mathbf{x}_{\sigma}-\mathbf{x}_{K}\right)+\mathbf{v}_{L}^{n} \cdot\left(\mathbf{x}_{L}-\mathbf{x}_{\sigma}\right)+\nu_{K} \mathrm{~m}(K) F_{K, \sigma}^{n}-\nu_{L} \mathrm{~m}(L) F_{L, \sigma}^{n}=p_{L}^{n}-p_{K}^{n}, \\
\forall \sigma=K \mid L \in \mathcal{E}_{\text {int }}, \forall n=1, \ldots, N_{k}, \\
F_{K, \sigma}^{n}+F_{L, \sigma}^{n}=0, \quad \forall \sigma=K \mid L \in \mathcal{E}_{\text {int }}, \forall n=1, \ldots, N_{k}, \\
\mathbf{U}_{K}^{n}=-A_{K}\left(c_{K}^{n-1}\right) \mathbf{v}_{K}^{n}, \quad \forall K \in \mathcal{M}, \forall n=1, \ldots, N_{k}, \\
\mathrm{~m}(K) \mathbf{U}_{K}^{n}=-\sum_{\sigma \in \mathcal{E}_{K}} F_{K, \sigma}^{n}\left(\mathbf{x}_{\sigma}-\mathbf{x}_{K}\right), \quad \forall K \in \mathcal{M}, \forall n=1, \ldots, N_{k}, \\
-\sum_{\sigma \in \mathcal{E}_{K}} F_{K, \sigma}^{n}=\operatorname{m}(K) q_{K}^{+, n}-\mathrm{m}(K) q_{K}^{-, n}, \quad \forall K \in \mathcal{M}, \forall n=1, \ldots, N_{k}, \\
\sum_{K \in \mathcal{M}} \mathrm{m}(K) p_{K}^{n}=0, \quad \forall n=1, \ldots, N_{k}, \\
F_{K, \sigma}^{n}=0, \quad \forall K \in \mathcal{M}, \forall \sigma \in \mathcal{E}_{K} \cap \mathcal{E}_{\text {ext }}, \forall n=1, \ldots, N_{k} .
\end{gathered}
$$

Denoting $\left(-F_{K, \sigma}^{n}\right)^{+}$and $\left(-F_{K, \sigma}^{n}\right)^{-}$the positive and negative parts of $-F_{K, \sigma}^{n}$, we discretize (1.8) in a similar fashion, using a time-implicit scheme (it is classical for parabolic equations):

$$
\begin{gathered}
\mathbf{w}_{K}^{n} \cdot\left(\mathbf{x}_{\sigma}-\mathbf{x}_{K}\right)+\mathbf{w}_{L}^{n} \cdot\left(\mathbf{x}_{L}-\mathbf{x}_{\sigma}\right)+\nu_{K} \mathrm{~m}(K) G_{K, \sigma}^{n}-\nu_{L} \mathrm{~m}(L) G_{L, \sigma}^{n}=c_{L}^{n}-c_{K}^{n}, \\
\forall \sigma=K \mid L \in \mathcal{E}_{\text {int }}, \forall n=1, \ldots, N_{k}, \\
G_{K, \sigma}^{n}+G_{L, \sigma}^{n}=0, \quad \forall \sigma=K \mid L \in \mathcal{E}_{\text {int }}, \forall n=1, \ldots, N_{k}, \\
\mathrm{~m}(K) D_{K}\left(\mathbf{U}_{K}^{n}\right) \mathbf{w}_{K}^{n}=\sum_{\sigma \in \mathcal{E}_{K}} G_{K, \sigma}^{n}\left(\mathbf{x}_{\sigma}-\mathbf{x}_{K}\right), \quad \forall K \in \mathcal{M}, \forall n=1, \ldots, N_{k}, \\
\mathrm{~m}(K) \Phi_{K} \frac{c_{K}^{n}-c_{K}^{n-1}}{k}-\sum_{\sigma \in \mathcal{E}_{K}} G_{K, \sigma}^{n}+\sum_{\sigma=K \mid L \in \mathcal{E}_{K} \cap \mathcal{E}_{\text {int }}}\left[\left(-F_{K, \sigma}^{n}\right)^{+} c_{K}^{n}-\left(-F_{K, \sigma}^{n}\right)^{-} c_{L}^{n}\right] \\
+\mathrm{m}(K) q_{K}^{-, n} c_{K}^{n}=\mathrm{m}(K) q_{K}^{+, n} \widehat{c}_{K}^{n}, \quad \forall K \in \mathcal{M}, \forall n=1, \ldots, N_{k}, \\
G_{K, \sigma}^{n}=0, \quad \forall K \in \mathcal{M}, \forall \sigma \in \mathcal{E}_{K} \cap \mathcal{E}_{\text {ext }}, \forall n=1, \ldots, N_{k} .
\end{gathered}
$$

Let us explain why each equation of this scheme is quite natural: 
- If we take $\nu_{K}=0,(2.2)$ and $(2.9)$ state that $\mathbf{v}_{K}^{n}(\approx \nabla \bar{p})$ and $\mathbf{w}_{K}^{n}(\approx \nabla \bar{c})$ are "discrete gradients" of $p_{K}^{n}(\approx \bar{p})$ and $c_{K}^{n}(\approx \bar{c})$; these are the discrete counterpart of $h\left(\mathbf{x}_{L}\right)-h\left(\mathbf{x}_{K}\right)=h\left(\mathbf{x}_{L}\right)-h\left(\mathbf{x}_{\sigma}\right)+$ $h\left(\mathbf{x}_{\sigma}\right)-h\left(\mathbf{x}_{K}\right) \approx \nabla h\left(\mathbf{x}_{L}\right) \cdot\left(\mathbf{x}_{L}-\mathbf{x}_{\sigma}\right)+\nabla h\left(\mathbf{x}_{K}\right) \cdot\left(\mathbf{x}_{\sigma}-\mathbf{x}_{K}\right)$ if $h$ is a regular function. The penalization using the fluxes (i.e. with $\nu_{K}>0$ ) is added to ensure the stability of the scheme.

- (2.3) and (2.10) state the conservativity of the fluxes, and are natural (because $\mathbf{n}_{K, \sigma}+\mathbf{n}_{L, \sigma}=0$ if $\left.\sigma=K \mid L \in \mathcal{E}_{\text {int }}\right)$ if one recalls that $F_{K, \sigma}^{n} \approx-\int_{\sigma} \overline{\mathbf{U}} \cdot \mathbf{n}_{K, \sigma} d \gamma$ and $G_{K, \sigma}^{n} \approx \int_{\sigma} D(\cdot, \overline{\mathbf{U}}) \nabla \bar{c} \cdot \mathbf{n}_{K, \sigma} d \gamma$.

- (2.8) and (2.13) translate the no flow boundary conditions.

- (2.6) and (2.12) come from the integration on a control volume and on a time step of the PDEs in (1.7) and (1.8). Notice that, as it is usual, we have chosen an upwind discretization for the convective term: $-F_{K, \sigma}^{n}$ is the flux outward $K$ associated with $\mathbf{U}_{K}^{n}$, and we see that $\left(-F_{K, \sigma}^{n}\right)^{+} c_{K}^{n}-$ $\left(-F_{K, \sigma}^{n}\right)^{-} c_{L}^{n}=-F_{K, \sigma}^{n} c_{K}^{n}$ if $-F_{K, \sigma}^{n} \geq 0$ and $\left(-F_{K, \sigma}^{n}\right)^{+} c_{K}^{n}-\left(-F_{K, \sigma}^{n}\right)^{-} c_{L}^{n}=-F_{K, \sigma}^{n} c_{L}^{n}$ if $-F_{K, \sigma}^{n} \leq 0$.

- (2.4) and (2.7) are straightforward expressions of the fact that $\overline{\mathbf{U}}=-A(\cdot, \bar{c}) \nabla \bar{p}$ and $\int_{\Omega} \bar{p}(t, \cdot)=0$.

- Lemma 7.1 in the appendix gives a formula that reconstructs a vector from its fluxes through the edges of a mesh. Since $F_{K, \sigma}^{n}$ and $G_{K, \sigma}^{n}$ are approximations of the fluxes of $-\overline{\mathbf{U}}$ and $D(\cdot, \overline{\mathbf{U}}) \nabla \bar{c}$, the formula in Lemma 7.1 justifies (2.5) and (2.11).

Remark 2.2 As explained in Section 4, the systems (2.2) -(2.8) and (2.9)-(2.13) are decoupled at each time step, which makes the computation of their solutions quite easy.

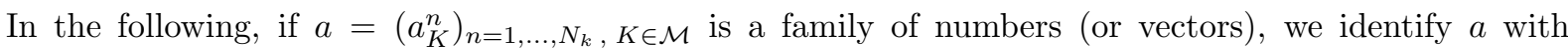
the function on $\left[0, T\left[\times \Omega\right.\right.$ which is constant equal to $a_{K}^{n}$ on each $[(n-1) k, n k[\times K$. Similarly, for $n$ fixed, $a^{n}=\left(a_{K}^{n}\right)_{K \in \mathcal{M}}$ is identified with the function on $\Omega$ which is constant equal to $a_{K}^{n}$ on each control volume $K$. Hence, for example, $p$ denotes both the family $\left(p_{K}^{n}\right)_{n=1, \ldots, N_{k}, K \in \mathcal{M}}$ and the corresponding function on $\left[0, T\left[\times \Omega\right.\right.$. We also denote $F$ and $G$ the families $\left(F_{K, \sigma}^{n}\right)_{n=1, \ldots, N_{k}, K \in \mathcal{M}, \sigma \in \mathcal{E}_{K}}$ and $\left(G_{K, \sigma}^{n}\right)_{n=1, \ldots, N_{k}}, K \in \mathcal{M}, \sigma \in \mathcal{E}_{K}$.

Our main results are the following theorems.

Theorem 2.1 Let $\Omega$ be a convex polygonal bounded open subset of $\mathbb{R}^{d}$ and $T>0$. Assume (1.9)-(1.14). Let $\mathcal{D}$ be an admissible discretization of $\Omega$ and $k>0$ such that $T / k$ is an integer. Then there exists a unique solution ( $p, \mathbf{v}, \mathbf{U}, F, c, \mathbf{w}, G)$ to (2.2)-(2.13).

Theorem 2.2 Let $\Omega$ be a convex polygonal bounded open subset of $\mathbb{R}^{d}$ and $T>0$. Assume (1.9)—(1.14). Let $\nu_{0}>0$ and $\left.\beta \in\right] 2-2 d, 4-2 d\left[\right.$. Let $\left(\mathcal{D}_{m}\right)_{m \geq 1}$ be a sequence of admissible discretizations of $\Omega$ such that $\operatorname{size}\left(\mathcal{D}_{m}\right) \rightarrow 0$ as $m \rightarrow \infty$ and $\left(\operatorname{regul}\left(\mathcal{D}_{m}\right)\right)_{m \geq 1}$ is bounded; we assume that there exists $C_{1}$ such that, for all $m \geq 1$,

$$
\forall(K, L) \in \mathcal{M}_{m} \times \mathcal{M}_{m} \text { neighboring control volumes, } \operatorname{diam}(K)^{2-\beta-d} \leq C_{1} \operatorname{diam}(L)^{d-2} .
$$

For all $K \in \mathcal{M}_{m}$, we take $\nu_{K}=\nu_{0} \operatorname{diam}(K)^{\beta}$. Let $k_{m}>0$ be such that $N_{k_{m}}=T / k_{m}$ is an integer and $k_{m} \rightarrow 0$ as $m \rightarrow \infty$, and denote $\left(p^{m}, \mathbf{v}^{m}, \mathbf{U}^{m}, F^{m}, c^{m}, \mathbf{w}^{m}, G^{m}\right)$ the solution to (2.2)-(2.13) with $\mathcal{D}=\mathcal{D}_{m}$ and $k=k_{m}$. Then, up to a subsequence, as $m \rightarrow \infty$,

$$
\begin{array}{ll}
p^{m} \rightarrow \bar{p} & \text { weakly-* in } L^{\infty}\left(0, T ; L^{2}(\Omega)\right) \text { and strongly in } L^{p}\left(0, T ; L^{q}(\Omega)\right) \text { for all } p<\infty \text { and all } q<2, \\
\mathbf{v}^{m} \rightarrow \nabla \bar{p} & \text { weakly-* in } L^{\infty}\left(0, T ; L^{2}(\Omega)\right)^{d} \text { and strongly in } L^{2}\left(10, T[\times \Omega)^{d},\right. \\
\mathbf{U}^{m} \rightarrow \overline{\mathbf{U}} & \text { weakly-* in } L^{\infty}\left(0, T ; L^{2}(\Omega)\right)^{d} \text { and strongly in } L^{2}(] 0, T[\times \Omega)^{d}, \\
c^{m} \rightarrow \bar{c} & \text { weakly-* in } L^{\infty}\left(0, T ; L^{2}(\Omega)\right) \text { and strongly in } L^{p}\left(0, T ; L^{q}(\Omega)\right) \text { for all } p<\infty \text { and all } q<2, \\
\mathbf{w}^{m} \rightarrow \nabla \bar{c} & \text { weakly in } L^{2}(] 0, T[\times \Omega)^{d},
\end{array}
$$

where $(\bar{p}, \overline{\mathbf{U}}, \bar{c})$ is a weak solution to $(1.7)-(1.8)$. 
Remark 2.3 As usual in finite volume schemes, we do not assume the existence of a solution to the continuous problem; this existence is obtained as a by-product of the proof of convergence. In particular, this means that, on the contrary to [5] or [11], the convergence of the mixed finite volume scheme is proved here under no regularity assumption on the solution to (1.7)-(1.8).

Remark 2.4 Note that, since $4-\beta-2 d \geq 0$, if

$\operatorname{diam}(K) \leq C_{2} \operatorname{diam}(L)$ for all neighboring control volumes $K$ and $L$ of a discretization $\mathcal{D}$,

then $\mathcal{D}$ also satisfies (2.14) with $C_{1}=C_{2}^{d-2} \operatorname{diam}(\Omega)^{4-\beta-2 d}$ if $d \geq 2$ and $C_{1}=C_{2} \operatorname{diam}(\Omega)^{2-\beta}$ if $d=1$. But (2.14) allows more freedom on the meshes:

- if $d=1$, choosing for example $\beta=1$, (2.14) only demands $\operatorname{diam}(L) \leq C_{1}$, which is always true with $C_{1}=\operatorname{diam}(\Omega)$.

- if $d=2$, (2.14) demands $\operatorname{diam}(K)^{-\beta} \leq C_{1}$, which is always satisfied with $C_{1}=\operatorname{diam}(\Omega)^{-\beta}$ since $\beta<0$ in this situation.

- if $d \geq 3$, choosing for example $\beta=3-2 d$, (2.14) reduces to $\operatorname{diam}(K) \leq C_{1}^{\frac{1}{d-1}} \operatorname{diam}(L)^{\frac{d-2}{d-1}}$, which permits, on the contrary to (2.15), local refinement procedures (since $\frac{d-2}{d-1}<1$ ).

\section{A priori estimates}

Proposition 3.1 Let $\Omega$ be a convex polygonal bounded open subset of $\mathbb{R}^{d}$ and $T>0$. Assume (1.9)(1.10). Let $\mathcal{D}$ be an admissible discretization of $\Omega$ such that $\operatorname{regul}(\mathcal{D}) \leq \theta$ for some $\theta>0$, and $k>0$ be such that $N_{k}=T / k$ is an integer. Let $\left(\nu_{K}\right)_{K \in \mathcal{M}}$ be a family of positive numbers such that, for some $\nu_{0}>0$ and $\beta \geq 2-2 d, \nu_{K} \leq \nu_{0} \operatorname{diam}(K)^{\beta}$ for all $K \in \mathcal{M}$. Then there exists $C_{3}$ only depending on $d$, $\Omega, \theta, \beta, \nu_{0}, \alpha_{A}$ and $\Lambda_{A}$ such that, for any numbers $\left(c_{K}^{n-1}\right)_{n=1, \ldots, N_{k}}, K \in \mathcal{M}$, any solution $(p, \mathbf{v}, \mathbf{U}, F)$ to (2.2)-(2.8) satisfies

$$
\begin{aligned}
\|p\|_{L^{\infty}\left(0, T ; L^{2}(\Omega)\right)}^{2}+\|\mathbf{v}\|_{L^{\infty}\left(0, T ; L^{2}(\Omega)\right)^{d}}^{2}+\|\mathbf{U}\|_{L^{\infty}\left(0, T ; L^{2}(\Omega)\right)^{d}}^{2}+\sup _{n=1, \ldots, N_{k}} \sum_{K \in \mathcal{M}} \sum_{\sigma \in \mathcal{E}_{K}} \nu_{K} \mathrm{~m}(K)\left|F_{K, \sigma}^{n}\right|^{2} \\
\leq C_{3}\left\|q^{+}-q^{-}\right\|_{L^{\infty}\left(0, T ; L^{2}(\Omega)\right)}^{2} .
\end{aligned}
$$

\section{Proof of Proposition 3.1}

Let $n \in\left[1, N_{k}\right]$. Multiply $(2.6)$ by $p_{K}^{n}$, sum on the control volumes and gather by edges using (2.3). Thanks to (2.8), the terms involving boundary edges disappear and this leads to

$$
\sum_{\sigma=K \mid L \in \mathcal{E}_{\text {int }}} F_{K, \sigma}^{n}\left(p_{L}^{n}-p_{K}^{n}\right)=\sum_{K \in \mathcal{M}} \mathrm{m}(K)\left(q_{K}^{+, n}-q_{K}^{-, n}\right) p_{K}^{n}=\int_{\Omega}\left(q^{+, n}-q^{-, n}\right) p^{n}
$$

where $q^{+, n}(\cdot)-q^{-, n}(\cdot)=\frac{1}{k} \int_{(n-1) k}^{n k} q^{+}(t, \cdot)-q^{-}(t, \cdot) d t$. Applying now (2.2) and gathering by control volumes (still using (2.3) and (2.8)), we deduce

$$
\begin{aligned}
\int_{\Omega}\left(q^{+, n}-q^{-, n}\right) p^{n}= & \sum_{\sigma=K \mid L \in \mathcal{E}_{\text {int }}} F_{K, \sigma}^{n}\left(\mathbf{v}_{K}^{n} \cdot\left(\mathbf{x}_{\sigma}-\mathbf{x}_{K}\right)+\mathbf{v}_{L}^{n} \cdot\left(\mathbf{x}_{L}-\mathbf{x}_{\sigma}\right)\right) \\
& +\sum_{\sigma=K \mid L \in \mathcal{E}_{\text {int }}} F_{K, \sigma}^{n}\left(\nu_{K} \mathrm{~m}(K) F_{K, \sigma}^{n}-\nu_{L} \mathrm{~m}(L) F_{L, \sigma}^{n}\right) \\
= & \sum_{K \in \mathcal{M}} \mathbf{v}_{K}^{n} \cdot \sum_{\sigma \in \mathcal{E}_{K}} F_{K, \sigma}^{n}\left(\mathbf{x}_{\sigma}-\mathbf{x}_{K}\right)+\sum_{K \in \mathcal{M}} \sum_{\sigma \in \mathcal{E}_{K}} \nu_{K} \mathrm{~m}(K)\left|F_{K, \sigma}^{n}\right|^{2} .
\end{aligned}
$$


Thanks to (2.5), (2.4) and hypothesis (1.10) (which implies that $A_{K}(s) \xi \cdot \xi \geq \alpha_{A}|\xi|^{2}$ for all $K \in \mathcal{M}$, all $s \in \mathbb{R}$ and all $\left.\xi \in \mathbb{R}^{d}\right)$, we find

$$
\begin{aligned}
\left\|q^{+, n}-q^{-, n}\right\|_{L^{2}(\Omega)}\left\|p^{n}\right\|_{L^{2}(\Omega)} & \geq \alpha_{A} \sum_{K \in \mathcal{M}} \mathrm{m}(K)\left|\mathbf{v}_{K}^{n}\right|^{2}+\sum_{K \in \mathcal{M}} \sum_{\sigma \in \mathcal{E}_{K}} \nu_{K} \mathrm{~m}(K)\left|F_{K, \sigma}^{n}\right|^{2} \\
& \geq \alpha_{A}\left\|\mathbf{v}^{n}\right\|_{L^{2}(\Omega)^{d}}^{2}+\sum_{K \in \mathcal{M}} \sum_{\sigma \in \mathcal{E}_{K}} \nu_{K} \mathrm{~m}(K)\left|F_{K, \sigma}^{n}\right|^{2} .
\end{aligned}
$$

We notice that (2.2) is exactly (7.1) for $\left(p^{n}, \mathbf{v}^{n}, F^{n}\right)$. Hence, since $p^{n}$ satisfies (2.7), we can apply the discrete Poincaré-Wirtinger inequality given in Lemma 7.2 and we have

$$
\left\|p^{n}\right\|_{L^{2}(\Omega)} \leq C_{4}\left(\left\|\mathbf{v}^{n}\right\|_{L^{2}(\Omega)^{d}}+\left(\sum_{K \in \mathcal{M}} \sum_{\sigma \in \mathcal{E}_{K}} \operatorname{diam}(K)^{2 d-2} \nu_{K}^{2} \mathrm{~m}(K)\left|F_{K, \sigma}^{n}\right|^{2}\right)^{\frac{1}{2}}\right)
$$

where $C_{4}$ only depends on $d, \Omega$ and $\theta$. By choice of $\nu_{K}$, we have $\operatorname{diam}(K)^{2 d-2} \nu_{K} \leq \nu_{0} \operatorname{diam}(K)^{2 d-2+\beta}$; but $2 d-2+\beta \geq 0$, and thus $\operatorname{diam}(K)^{2 d-2} \nu_{K} \leq \nu_{0} \operatorname{diam}(\Omega)^{2 d-2+\beta}$. Hence

$$
\left\|p^{n}\right\|_{L^{2}(\Omega)} \leq C_{5}\left(\left\|\mathbf{v}^{n}\right\|_{L^{2}(\Omega)^{d}}+\left(\sum_{K \in \mathcal{M}} \sum_{\sigma \in \mathcal{E}_{K}} \nu_{K} \mathrm{~m}(K)\left|F_{K, \sigma}^{n}\right|^{2}\right)^{\frac{1}{2}}\right)
$$

where $C_{5}$ only depends on $d, \Omega, \theta, \beta$ and $\nu_{0}$. Using this in (3.2), we obtain

$$
\begin{aligned}
& \alpha_{A}\left\|\mathbf{v}^{n}\right\|_{L^{2}(\Omega)^{d}}^{2}+\sum_{K \in \mathcal{M}} \sum_{\sigma \in \mathcal{E}_{K}} \nu_{K} \mathrm{~m}(K)\left|F_{K, \sigma}^{n}\right|^{2} \\
& \quad \leq C_{5}\left\|q^{+, n}-q^{-, n}\right\|_{L^{2}(\Omega)}\left\|\mathbf{v}^{n}\right\|_{L^{2}(\Omega)^{d}}+C_{5}\left\|q^{+, n}-q^{-, n}\right\|_{L^{2}(\Omega)}\left(\sum_{K \in \mathcal{M}} \sum_{\sigma \in \mathcal{E}_{K}} \nu_{K} \mathrm{~m}(K)\left|F_{K, \sigma}^{n}\right|^{2}\right)^{\frac{1}{2}} .
\end{aligned}
$$

Using Young's inequality, this gives the desired bound on $\mathbf{v}$ and $F$ and, coming back to (3.3), the bound on $p$. The bound on $\mathbf{U}$ derives from the one on $\mathbf{v}$, since $A$ is bounded (see (1.10)).

Proposition 3.2 Let $\Omega$ be a convex polygonal bounded open subset of $\mathbb{R}^{d}$ and $T>0$. Assume (1.9) and (1.11) -(1.14). Let $\mathcal{D}$ be an admissible discretization of $\Omega$, and $k>0$ be such that $N_{k}=T / k$ is an integer. Let $\left(\nu_{K}\right)_{K \in \mathcal{M}}$ be a family of positive numbers. Assume that $F=\left(F_{K, \sigma}^{n}\right)_{n=1, \ldots, N_{k}}, K \in \mathcal{M}, \sigma \in \mathcal{E}_{K}$

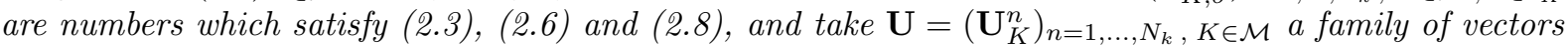
in $\mathbb{R}^{d}$. Then there exists $C_{6}$ only depending on $d, \Omega, T, \alpha_{D}$ and $\Phi_{*}$ such that any solution $(c, \mathbf{w}, G)$ to (2.9)-(2.13) satisfies

$$
\begin{gathered}
\|c\|_{L^{\infty}\left(0, T ; L^{2}(\Omega)\right)}^{2}+\|\mathbf{w}\|_{L^{2}(] 0, T[\times \Omega)^{d}}^{2}+\left\||\mathbf{U}|^{1 / 2}|\mathbf{w}|\right\|_{L^{2}(] 0, T[\times \Omega)}^{2}+\sum_{n=1}^{N_{k}} k \sum_{K \in \mathcal{M}} \sum_{\sigma \in \mathcal{E}_{K}} \nu_{K} \mathrm{~m}(K)\left|G_{K, \sigma}^{n}\right|^{2} \\
\leq C_{6}\left\|c_{0}\right\|_{L^{2}(\Omega)}^{2}+C_{6}\left\|q^{+}\right\|_{L^{\infty}\left(0, T ; L^{2}(\Omega)\right)}^{2} .
\end{gathered}
$$

\section{Proof of Proposition 3.2}

Multiply (2.12) by $c_{K}^{n}$ and sum on the control volumes. We have

$$
(a-b) a=a^{2}-a b=\frac{1}{2}\left(a^{2}-b^{2}\right)+\frac{1}{2}(a-b)^{2} \geq \frac{1}{2}\left(a^{2}-b^{2}\right)
$$


and therefore $\left(c_{K}^{n}-c_{K}^{n-1}\right) c_{K}^{n} \geq \frac{1}{2}\left(c_{K}^{n}\right)^{2}-\frac{1}{2}\left(c_{K}^{n-1}\right)^{2}$. Hence, using (2.10) to gather by edges (dropping the boundary edges thanks to (2.13)), and since $\Phi_{K} \geq 0$, we obtain

$$
\begin{aligned}
& \frac{1}{2 k} \sum_{K \in \mathcal{M}} \mathrm{m}(K) \Phi_{K}\left(\left(c_{K}^{n}\right)^{2}-\left(c_{K}^{n-1}\right)^{2}\right)+\sum_{\sigma=K \mid L \in \mathcal{E}_{\mathrm{int}}} G_{K, \sigma}^{n}\left(c_{L}^{n}-c_{K}^{n}\right) \\
& +\sum_{K \in \mathcal{M}} \sum_{\sigma=K \mid L \in \mathcal{E}_{K} \cap \mathcal{E}_{\mathrm{int}}}\left[\left(-F_{K, \sigma}^{n}\right)^{+} c_{K}^{n}-\left(-F_{K, \sigma}^{n}\right)^{-} c_{L}^{n}\right] c_{K}^{n}+\sum_{K \in \mathcal{M}} \mathrm{m}(K) q_{K}^{-, n}\left(c_{K}^{n}\right)^{2} \\
& \leq \sum_{K \in \mathcal{M}} \mathrm{m}(K)\left|q_{K}^{+, n} \widehat{c}_{K}^{n}\right|\left|c_{K}^{n}\right| .
\end{aligned}
$$

Let us handle the third term of this inequality. We have, gathering by edges and using (2.3) (which implies $\left(-F_{K, \sigma}^{n}\right)^{+}=\left(-F_{L, \sigma}^{n}\right)^{-}$whenever $\left.\sigma=K \mid L \in \mathcal{E}_{\text {int }}\right)$,

$$
\begin{aligned}
\sum_{K \in \mathcal{M}} & \sum_{\sigma=K \mid L \in \mathcal{E}_{K} \cap \mathcal{E}_{\text {int }}}\left[\left(-F_{K, \sigma}^{n}\right)^{+} c_{K}^{n}-\left(-F_{K, \sigma}^{n}\right)^{-} c_{L}^{n}\right] c_{K}^{n} \\
= & \sum_{\sigma=K \mid L \in \mathcal{E}_{\text {int }}}\left[\left(-F_{K, \sigma}^{n}\right)^{+}\left(c_{K}^{n}\right)^{2}-\left(-F_{K, \sigma}^{n}\right)^{-} c_{L}^{n} c_{K}^{n}+\left(-F_{L, \sigma}^{n}\right)^{+}\left(c_{L}^{n}\right)^{2}-\left(-F_{L, \sigma}^{n}\right)^{-} c_{K}^{n} c_{L}^{n}\right] \\
= & \left.\sum_{\sigma=K \mid L \in \mathcal{E}_{\text {int }}}\left[\left(-F_{K, \sigma}^{n}\right)^{+}\left(\left(c_{K}^{n}\right)^{2}-c_{K}^{n} c_{L}^{n}\right)\right)+\left(-F_{L, \sigma}^{n}\right)^{+}\left(\left(c_{L}^{n}\right)^{2}-c_{L}^{n} c_{K}^{n}\right)\right] .
\end{aligned}
$$

Recalling (3.4), we continue with

$$
\begin{aligned}
\sum_{K \in \mathcal{M}} \sum_{\sigma=K \mid L \in \mathcal{E}_{K} \cap \mathcal{E}_{\mathrm{int}}}\left[\left(-F_{K, \sigma}^{n}\right)^{+} c_{K}^{n}-\left(-F_{K, \sigma}^{n}\right)^{-} c_{L}^{n}\right] c_{K}^{n} \\
\left.\geq \frac{1}{2} \sum_{\sigma=K \mid L \in \mathcal{E}_{\mathrm{int}}}\left[\left(-F_{K, \sigma}^{n}\right)^{+}\left(\left(c_{K}^{n}\right)^{2}-\left(c_{L}^{n}\right)^{2}\right)\right)+\left(-F_{L, \sigma}^{n}\right)^{+}\left(\left(c_{L}^{n}\right)^{2}-\left(c_{K}^{n}\right)^{2}\right)\right] \\
\geq \frac{1}{2} \sum_{\sigma=K \mid L \in \mathcal{E}_{\mathrm{int}}}\left[\left(-F_{K, \sigma}^{n}\right)^{+}-\left(-F_{K, \sigma}^{n}\right)^{-}\right]\left(\left(c_{K}^{n}\right)^{2}-\left(c_{L}^{n}\right)^{2}\right) \\
\geq \frac{1}{2} \sum_{\sigma=K \mid L \in \mathcal{E}_{\mathrm{int}}}-F_{K, \sigma}^{n}\left(\left(c_{K}^{n}\right)^{2}-\left(c_{L}^{n}\right)^{2}\right)
\end{aligned}
$$

which gives, gathering by control volumes and using (2.3), (2.8) and (2.6),

$$
\begin{aligned}
\sum_{K \in \mathcal{M}} \sum_{\sigma=K \mid L \in \mathcal{E}_{K} \cap \mathcal{E}_{\mathrm{int}}}\left[\left(-F_{K, \sigma}^{n}\right)^{+} c_{K}^{n}-\left(-F_{K, \sigma}^{n}\right)^{-} c_{L}^{n}\right] c_{K}^{n} & \geq \frac{1}{2} \sum_{K \in \mathcal{M}}\left(c_{K}^{n}\right)^{2}\left(-\sum_{\sigma \in \mathcal{E}_{K}} F_{K, \sigma}^{n}\right) \\
& \geq \frac{1}{2} \sum_{K \in \mathcal{M}} \mathrm{m}(K)\left(c_{K}^{n}\right)^{2}\left(q_{K}^{+, n}-q_{K}^{-, n}\right) .
\end{aligned}
$$

Since

$$
\frac{1}{2} \sum_{K \in \mathcal{M}} \mathrm{m}(K)\left(c_{K}^{n}\right)^{2}\left(q_{K}^{+, n}-q_{K}^{-, n}\right)+\sum_{K \in \mathcal{M}} \mathrm{m}(K) q_{K}^{-, n}\left(c_{K}^{n}\right)^{2}=\frac{1}{2} \sum_{K \in \mathcal{M}} \mathrm{m}(K)\left(q_{K}^{+, n}+q_{K}^{-, n}\right)\left(c_{K}^{n}\right)^{2} \geq 0
$$

(because $q^{+}$and $q^{-}$are nonnegative), we deduce from (3.5) that

$$
\frac{1}{2 k} \sum_{K \in \mathcal{M}} \mathrm{m}(K) \Phi_{K}\left(\left(c_{K}^{n}\right)^{2}-\left(c_{K}^{n-1}\right)^{2}\right)+\sum_{\sigma=K \mid L \in \mathcal{E}_{\text {int }}} G_{K, \sigma}^{n}\left(c_{L}^{n}-c_{K}^{n}\right) \leq \sum_{K \in \mathcal{M}} \mathrm{m}(K)\left|q_{K}^{+, n} \widehat{c}_{K}^{n}\right|\left|c_{K}^{n}\right| .
$$


We now handle the second term of this equation. Thanks to (2.9), (2.10), (2.13) and (2.11), we have

$$
\begin{aligned}
\sum_{\sigma=K \mid L \in \mathcal{E}_{\text {int }}} G_{K, \sigma}^{n}\left(c_{L}^{n}-c_{K}^{n}\right)= & \sum_{\sigma=K \mid L \in \mathcal{E}_{\text {int }}} G_{K, \sigma}^{n}\left(\mathbf{w}_{K}^{n} \cdot\left(\mathbf{x}_{\sigma}-\mathbf{x}_{K}\right)+\mathbf{w}_{L}^{n} \cdot\left(\mathbf{x}_{L}-\mathbf{x}_{\sigma}\right)\right) \\
& +\sum_{\sigma=K \mid L \in \mathcal{E}_{\text {int }}} G_{K, \sigma}^{n}\left(\nu_{K} \mathrm{~m}(K) G_{K, \sigma}^{n}-\nu_{L} \mathrm{~m}(L) G_{L, \sigma}^{n}\right) \\
= & \sum_{K \in \mathcal{M}} \mathbf{w}_{K}^{n} \cdot \sum_{\sigma \in \mathcal{E}_{K}} G_{K, \sigma}^{n}\left(\mathbf{x}_{\sigma}-\mathbf{x}_{K}\right)+\sum_{K \in \mathcal{M}} \sum_{\sigma \in \mathcal{E}_{K}} \nu_{K} \mathrm{~m}(K)\left|G_{K, \sigma}^{n}\right|^{2} \\
= & \sum_{K \in \mathcal{M}} \mathrm{m}(K) D_{K}\left(\mathbf{U}_{K}^{n}\right) \mathbf{w}_{K}^{n} \cdot \mathbf{w}_{K}^{n}+\sum_{K \in \mathcal{M}} \sum_{\sigma \in \mathcal{E}_{K}} \nu_{K} \mathrm{~m}(K)\left|G_{K, \sigma}^{n}\right|^{2} .
\end{aligned}
$$

We then use (1.11) and inject the corresponding lower bound in (3.6), which we multiply by $k$ and sum on $n=1, \ldots, N$ (for some $N \in\left[1, N_{k}\right]$ ); since $|\widehat{c}| \leq 1$, this leads to

$$
\begin{aligned}
\frac{1}{2} \sum_{K \in \mathcal{M}} \mathrm{m}(K) \Phi_{K}\left(\left(c_{K}^{N}\right)^{2}-\left(c_{K}^{0}\right)^{2}\right)+\alpha_{D} \sum_{n=1}^{N} k & \sum_{K \in \mathcal{M}} \mathrm{m}(K)\left(1+\left|\mathbf{U}_{K}^{n}\right|\right)\left|\mathbf{w}_{K}^{n}\right|^{2} \\
+\sum_{n=1}^{N} k \sum_{K \in \mathcal{M}} \sum_{\sigma \in \mathcal{E}_{K}} \nu_{K} \mathrm{~m}(K)\left|G_{K, \sigma}^{n}\right|^{2} & \leq \int_{0}^{T} \int_{\Omega}\left|q^{+}\right||c| \\
& \leq\left. T|| q^{+}\right|_{L^{\infty}\left(0, T ; L^{2}(\Omega)\right)}|| c||_{L^{\infty}\left(0, T ; L^{2}(\Omega)\right)} .
\end{aligned}
$$

This gives in particular, by (1.12) and the definition of $\left(c_{K}^{0}\right)_{K \in \mathcal{M}}$,

$$
\frac{\Phi_{*}}{2} \sum_{K \in \mathcal{M}} \mathrm{m}(K)\left(c_{K}^{N}\right)^{2} \leq \frac{\Phi_{*}^{-1}}{2}\left\|c_{0}\right\|_{L^{2}(\Omega)}^{2}+\frac{T^{2}}{\Phi_{*}}\left\|q^{+}\right\|_{L^{\infty}\left(0, T ; L^{2}(\Omega)\right)}^{2}+\frac{\Phi_{*}}{4}\|c\|_{L^{\infty}\left(0, T ; L^{2}(\Omega)\right)}^{2}
$$

Since $\|c\|_{L^{\infty}\left(0, T ; L^{2}(\Omega)\right)}^{2}=\sup _{r=1, \ldots, N_{k}} \sum_{K \in \mathcal{M}} \mathrm{m}(K)\left(c_{K}^{r}\right)^{2}$, this inequality, valid for all $N=1, \ldots, N_{k}$, gives the estimate on $\|c\|_{L^{\infty}\left(0, T ; L^{2}(\Omega)\right)}$. Used back in (3.8), this estimate gives the desired bounds on $\mathbf{w}$, $|\mathbf{U}|^{1 / 2}|\mathbf{w}|$ and $G$.

\section{Existence, uniqueness and computation of the solution to the scheme}

\subsection{Proof of Theorem 2.1}

Note first that, solving the equations one time step after the other, (2.2) - (2.8) and (2.9) - (2.13) are decoupled: at time step $n$, the knowledge of $c_{K}^{n-1}$ (from the definition of $c_{K}^{0}$ if $n=1$, or the preceding time step if $n \geq 2)$ shows that $(2.2)-(2.8)$ is a linear system on $\left(p^{n}, \mathbf{v}^{n}, \mathbf{U}^{n},\left(F_{K, \sigma}^{n}\right)_{K \in \mathcal{M}, \sigma \in \mathcal{E}_{K}}\right)$ alone; once this system is solved, $\mathbf{U}^{n}$ is known and (2.9)-(2.13) becomes a linear system on $\left(c^{n}, \mathbf{w}^{n},\left(G_{K, \sigma}^{n}\right)_{K \in \mathcal{M}, \sigma \in \mathcal{E}_{K}}\right)$ alone. Hence, to prove Theorem 2.1 we only need to show that, at each time step, there exists one and only one solution to each of these linear systems.

Let us first consider the system on $\left(c^{n}, \mathbf{w}^{n},\left(G_{K, \sigma}^{n}\right)_{K \in \mathcal{M}, \sigma \in \mathcal{E}_{K}}\right)$. By (2.10) and (2.13), we can consider that there is only one flux by interior edge and this system therefore has $(d+1) \operatorname{Card}(\mathcal{M})+\operatorname{Card}\left(\mathcal{E}_{\text {int }}\right)$ unknowns, with as many remaining equations $((2.11)$ gives $d \operatorname{Card}(\mathcal{M})$ equations, $(2.12)$ another $\operatorname{Card}(\mathcal{M})$ equations and $(2.9)$ the last $\operatorname{Card}\left(\mathcal{E}_{\text {int }}\right)$ equations). Hence, this first system is square. Assume that $\left(c^{n}, \mathbf{w}^{n},\left(G_{K, \sigma}^{n}\right)_{K \in \mathcal{M}, \sigma \in \mathcal{E}_{K}}\right)$ is a solution with a null right-hand side, i.e. with $c^{n-1}=\widehat{c}^{n}=0$; then (3.6) and (3.7) show that this solution is null, and therefore that this system is invertible.

Without (2.7), and since we can eliminate $\mathbf{U}^{n}$ by (2.4), the system on $\left(p^{n}, \mathbf{v}^{n}, \mathbf{U}^{n},\left(F_{K, \sigma}^{n}\right)_{K \in \mathcal{M}, \sigma \in \mathcal{E}_{K}}\right)$ also has $(d+1) \operatorname{Card}(\mathcal{M})+\operatorname{Card}\left(\mathcal{E}_{\text {int }}\right)$ unknowns and the same number of equations. However, it is not invertible 
since its kernel clearly contains $(C, 0,0,0)$ (where $C=(C)_{K \in \mathcal{M}}$ is any constant vector); in fact, the estimates in the preceding section show that these vectors fully describe the kernel of $((2.2)-(2.6),(2.8))$ : if $\left(p^{n}, \mathbf{v}^{n}, \mathbf{U}^{n},\left(F_{K, \sigma}^{n}\right)_{K \in \mathcal{M}, \sigma \in \mathcal{E}_{K}}\right)$ belongs to this kernel, then $\left(p^{n}-C, \mathbf{v}^{n}, \mathbf{U}^{n},\left(F_{K, \sigma}^{n}\right)_{K \in \mathcal{M}, \sigma \in \mathcal{E}_{K}}\right)$, where $C$ is a constant vector such that $(2.7)$ holds with $p^{n}-C$, satisfies $(2.2)-(2.8)$ with $q^{+, n}-q^{-, n}=0$, and is therefore null by the estimate in Proposition 3.1, which shows that $\left(p^{n}, \mathbf{v}^{n}, \mathbf{U}^{n},\left(F_{K, \sigma}^{n}\right)_{\left.K \in \mathcal{M}, \sigma \in \mathcal{E}_{K}\right)=}\right.$ $(C, 0,0,0)$.

Summing (2.6) on $K$ and using $(2.3)$ and (2.8), we see that a necessary condition for $((2.2)-(2.6),(2.8))$ to have a solution is

$$
\sum_{K \in \mathcal{M}} \mathrm{m}(K) q_{K}^{n,+}-\mathrm{m}(K) q_{K}^{n,-}=0
$$

Since the kernel of this square system $((2.2)-(2.6),(2.8))$ has dimension 1 (and thus the range of the corresponding matrix has codimension 1), this condition is also sufficient, and is clearly satisfied by the data we consider thanks to (1.9). We can therefore always find a solution to $((2.2)-(2.6),(2.8))$ and, in view of the kernel of this system, (2.7) then selects one and only one solution.

\subsection{Hybridation}

As said above, at each time step the scheme (2.2)-(2.13) can be decoupled in two successive linear systems, $(2.2)-(2.8)$ and then $(2.9)-(2.13)$, each one with size $(d+1) \operatorname{Card}(\mathcal{M})+\operatorname{Card}\left(\mathcal{E}_{\text {int }}\right)$. However, it is possible to proceed to an algebraic elimination which leads to smaller sparse linear systems, following [18] for the mixed finite element method and [9] for the mixed finite volume method for anisotropic diffusion problems.

Let us consider the time step $n$ : $c^{n-1}$ is known (either from the preceding time step, or from the definition of $\left.\left(c_{K}^{0}\right)_{K \in \mathcal{M}}\right)$ and we present how to compute $\left(p^{n}, \mathbf{v}^{n}, \mathbf{U}^{n}, F^{n}, c^{n}, \mathbf{w}^{n}, G^{n}\right)$. We let drop the superscript $n$, except for $c^{n-1}$ which corresponds to the "initial condition" of the time step.

\section{Computation of $(p, \mathbf{v}, \mathbf{U}, F)$}

We introduce the auxiliary vector $\left(p_{\sigma}\right)_{\sigma \in \mathcal{E}_{\text {int }}}$ defined by:

$$
\mathbf{v}_{K} \cdot\left(\mathbf{x}_{\sigma}-\mathbf{x}_{K}\right)+\nu_{K} \mathrm{~m}(K) F_{K, \sigma}=p_{\sigma}-p_{K}, \quad \forall \sigma \in \mathcal{E}_{K} \cap \mathcal{E}_{\text {int }}, \quad \forall K \in \mathcal{M}
$$

Using (2.4), (2.5) and (2.8), we get:

$$
\begin{gathered}
\frac{1}{\mathrm{~m}(K)} \sum_{\sigma^{\prime} \in \mathcal{E}_{K} \cap \mathcal{E}_{\mathrm{int}}}\left(A_{K}\left(c_{K}^{n-1}\right)\right)^{-1}\left(\mathbf{x}_{\sigma^{\prime}}-\mathbf{x}_{K}\right) \cdot\left(\mathbf{x}_{\sigma}-\mathbf{x}_{K}\right) F_{K, \sigma^{\prime}}+\nu_{K} \mathrm{~m}(K) F_{K, \sigma}=p_{\sigma}-p_{K}, \\
\forall \sigma \in \mathcal{E}_{K} \cap \mathcal{E}_{\text {int }}, \forall K \in \mathcal{M} .
\end{gathered}
$$

This means that, for all $K \in \mathcal{M},\left(F_{K, \sigma}\right)_{\sigma \in \mathcal{E}_{K} \cap \mathcal{E}_{\text {int }}}$ is the solution to a linear system $B_{K}\left(F_{K, \sigma}\right)_{\sigma \in \mathcal{E}_{K} \cap \mathcal{E}_{\text {int }}=}=$

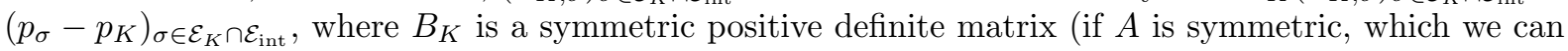
assume for physical problems - notice that the hybridation we present here is also valid in the case where $A$ is not symmetric). The resolution of this small local linear system leads to:

$$
F_{K, \sigma}=\sum_{\sigma^{\prime} \in \mathcal{E}_{K} \cap \mathcal{E}_{\mathrm{int}}}\left(B_{K}^{-1}\right)_{\sigma \sigma^{\prime}}\left(p_{\sigma^{\prime}}-p_{K}\right), \quad \forall \sigma \in \mathcal{E}_{K} \cap \mathcal{E}_{\mathrm{int}}, \forall K \in \mathcal{M}
$$

We set $b_{K, \sigma^{\prime}}=\sum_{\sigma \in \mathcal{E}_{K} \cap \mathcal{E}_{\text {int }}}\left(B_{K}^{-1}\right)_{\sigma \sigma^{\prime}}$ and $b_{K}=\sum_{\sigma^{\prime} \in \mathcal{E}_{K} \cap \mathcal{E}_{\text {int }}} b_{K, \sigma^{\prime}}=B_{K}^{-1}(1)_{\sigma \in \mathcal{E}_{K} \cap \mathcal{E}_{\text {int }}} \cdot(1)_{\sigma \in \mathcal{E}_{K} \cap \mathcal{E}_{\text {int }}}>0$. Reporting (4.2) in (2.6), we get:

$$
p_{K}=\frac{1}{b_{K}}\left(\mathrm{~m}(K)\left(q_{K}^{+}-q_{K}^{-}\right)+\sum_{\sigma^{\prime} \in \mathcal{E}_{K} \cap \mathcal{E}_{\mathrm{int}}} b_{K, \sigma^{\prime}} p_{\sigma^{\prime}}\right) .
$$


We can then report (4.3) in (4.2) and write the conservativity equation (2.3). It yields:

$$
\begin{gathered}
\sum_{\sigma^{\prime} \in \mathcal{E}_{K} \cap \mathcal{E}_{\text {int }}}\left(\left(B_{K}^{-1}\right)_{\sigma \sigma^{\prime}}-\frac{b_{K, \sigma^{\prime}} b_{K, \sigma}}{b_{K}}\right) p_{\sigma^{\prime}}+\sum_{\sigma^{\prime} \in \mathcal{E}_{L} \cap \mathcal{E}_{\text {int }}}\left(\left(B_{L}^{-1}\right)_{\sigma \sigma^{\prime}}-\frac{b_{L, \sigma^{\prime}} b_{L, \sigma}}{b_{L}}\right) p_{\sigma^{\prime}}= \\
\frac{b_{K, \sigma}}{b_{K}} \mathrm{~m}(K)\left(q_{K}^{+}-q_{K}^{-}\right)+\frac{b_{L, \sigma}}{b_{L}} \mathrm{~m}(L)\left(q_{L}^{+}-q_{L}^{-}\right), \quad \forall \sigma=K \mid L \in \mathcal{E}_{\text {int }} .
\end{gathered}
$$

This is a symmetric linear system, whose unknowns are $\left(p_{\sigma}\right)_{\sigma \in \mathcal{E}_{\text {int }}}$. Its matrix $M$ is positive but is not invertible because we have not used, up to this point, the relation (2.7). Thanks to (4.3), this relation writes:

$$
\sum_{K \in \mathcal{M}} \sum_{\sigma^{\prime} \in \mathcal{E}_{K} \cap \mathcal{E}_{\mathrm{int}}} \frac{b_{K, \sigma^{\prime}}}{b_{K}} \mathrm{~m}(K) p_{\sigma^{\prime}}=-\sum_{K \in \mathcal{M}} \frac{\mathrm{m}(K)}{b_{K}} \mathrm{~m}(K)\left(q_{K}^{+}-q_{K}^{-}\right) .
$$

It is clear that, with (4.3) and (4.2), the linear system (4.4)-(4.5) on $\left(p_{\sigma}\right)_{\sigma \in \mathcal{E}_{\text {int }}}$ is equivalent to the linear system $(2.2)-(2.8)$ on $(p, \mathbf{v}, \mathbf{U}, F)$; therefore (4.4)-(4.5) always admits a unique solution, from which we can compute $p, F, \mathbf{U}$ and $\mathbf{v}$ (using (4.3), (4.2), (2.5) and (2.4)). The computation of $(p, \mathbf{v}, \mathbf{U}, F)$ thus reduces to the resolution of a linear system with $\operatorname{Card}\left(\mathcal{E}_{\text {int }}\right)$ unknowns.

\section{Computation of $(c, \mathbf{w}, G)$}

We apply the same method for the computation of $(c, \mathbf{w}, G)$. First, we introduce the auxiliary vector $\left(c_{\sigma}\right)_{\sigma \in \mathcal{E}_{\text {int }}}$ defined by:

$$
\mathbf{w}_{K} \cdot\left(\mathbf{x}_{\sigma}-\mathbf{x}_{K}\right)+\nu_{K} \mathrm{~m}(K) G_{K, \sigma}=c_{\sigma}-c_{K}, \quad \forall \sigma \in \mathcal{E}_{K} \cap \mathcal{E}_{\text {int }}, \forall K \in \mathcal{M} .
$$

Using (2.11) and (2.13), we see that, for all $K \in \mathcal{M},\left(G_{K, \sigma}\right)_{\sigma \in \mathcal{E}_{K} \cap \mathcal{E}_{\text {int }}}$ is solution to a linear system

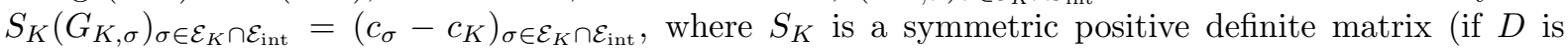
symmetric). The resolution of this small local linear system leads to:

$$
G_{K, \sigma}=\sum_{\sigma^{\prime} \in \mathcal{E}_{K} \cap \mathcal{E}_{\text {int }}}\left(S_{K}^{-1}\right)_{\sigma \sigma^{\prime}}\left(c_{\sigma^{\prime}}-c_{K}\right), \quad \forall \sigma \in \mathcal{E}_{K} \cap \mathcal{E}_{\text {int }}, \forall K \in \mathcal{M}
$$

and then

$$
\sum_{\sigma \in \mathcal{E}_{K} \cap \mathcal{E}_{\mathrm{int}}} G_{K, \sigma}=\left(\sum_{\sigma^{\prime} \in \mathcal{E}_{K} \cap \mathcal{E}_{\mathrm{int}}} s_{K, \sigma^{\prime}} c_{\sigma^{\prime}}\right)-s_{K} c_{K},
$$

with $s_{K, \sigma^{\prime}}=\sum_{\sigma \in \mathcal{E}_{K} \cap \mathcal{E}_{\text {int }}}\left(S_{K}^{-1}\right)_{\sigma \sigma^{\prime}}$ and $s_{K}=\sum_{\sigma^{\prime} \in \mathcal{E}_{K} \cap \mathcal{E}_{\text {int }}} s_{K, \sigma^{\prime}}$. Reporting this in (2.12), we get

$$
\begin{gathered}
\mathrm{m}(K) \Phi_{K} \frac{c_{K}}{k}+s_{K} c_{K}+\sum_{\sigma=K \mid L \in \mathcal{E}_{K} \cap \mathcal{E}_{\text {int }}}\left[\left(-F_{K, \sigma}\right)^{+} c_{K}-\left(-F_{K, \sigma}\right)^{-} c_{L}\right]+\mathrm{m}(K) q_{K}^{-} c_{K}= \\
\mathrm{m}(K) \Phi_{K} \frac{c_{K}^{n-1}}{k}+\mathrm{m}(K) q_{K}^{+} \widehat{c}_{K}+\sum_{\sigma^{\prime} \in \mathcal{E}_{K} \cap \mathcal{E}_{\text {int }}} s_{K, \sigma^{\prime}} c_{\sigma^{\prime}}, \quad \forall K \in \mathcal{M} .
\end{gathered}
$$

Injecting (4.6) in the conservativity relation (2.10), we have:

$$
\sum_{\sigma^{\prime} \in \mathcal{E}_{K} \cap \mathcal{E}_{\mathrm{int}}}\left(S_{K}^{-1}\right)_{\sigma \sigma^{\prime}}\left(c_{\sigma^{\prime}}-c_{K}\right)+\sum_{\sigma^{\prime} \in \mathcal{E}_{L} \cap \mathcal{E}_{\mathrm{int}}}\left(S_{L}^{-1}\right)_{\sigma \sigma^{\prime}}\left(c_{\sigma^{\prime}}-c_{L}\right)=0, \quad \forall \sigma=K \mid L \in \mathcal{E}_{\mathrm{int}} .
$$

Finally, (4.7)-(4.8) gives us an invertible (because it is equivalent to (2.9)-(2.13)) linear system on $\left(\left(c_{K}\right)_{K \in \mathcal{M}},\left(c_{\sigma}\right)_{\sigma \in \mathcal{E}_{\text {int }}}\right)$, with size $\operatorname{Card}(\mathcal{M})+\operatorname{Card}\left(\mathcal{E}_{\text {int }}\right)$. The knowledge of $\left(\left(c_{K}\right)_{K \in \mathcal{M}},\left(c_{\sigma}\right)_{\sigma \in \mathcal{E}_{\text {int }}}\right)$ allows to find $G$ as a consequence of (4.6) and $\mathbf{w}$ as a consequence of (2.11).

Remark 4.1 The size of the linear system cannot be reduced to $\operatorname{Card}\left(\mathcal{E}_{\mathrm{int}}\right)$ because of the implicit discretization of the convective term $\operatorname{div}(c \mathbf{U})$. If we use an explicit discretization for this term, then (4.7) permits to immediately write $c_{K}$ as a combination of the $\left(c_{\sigma^{\prime}}\right)_{\sigma^{\prime} \in \mathcal{E}_{K} \cap \mathcal{E}_{\mathrm{int}}}$ (and of known quantities), which can be used in (4.8) and reduces the whole procedure to a system on $\left(c_{\sigma^{\prime}}\right)_{\sigma^{\prime} \in \mathcal{E}_{\text {int }}}$ of size $\operatorname{Card}\left(\mathcal{E}_{\mathrm{int}}\right)$. 


\section{Proof of the convergence of the scheme}

In this section, we prove Theorem 2.2. To simplify the notations, we drop the index $m$ and we thus prove the desired convergence as $\operatorname{size}(\mathcal{D}) \rightarrow 0$ and $k \rightarrow 0$, with $\operatorname{regul}(\mathcal{D})$ bounded and $(2.14)$ uniformly satisfied for all considered discretizations. Under these assumptions, Propositions 3.1 and 3.2 give estimates which are uniform with respect to the discretizations and time steps.

\subsection{Compactness of the concentration}

Lemma 5.1 Under the assumptions of Theorem 2.2, $c$ is relatively compact in $L^{1}\left(0, T ; L_{\mathrm{loc}}^{1}(\Omega)\right)$.

Proof of Lemma 5.1

The proof is made in two steps. First we construct an affine interpolant $\widetilde{c}$ of $c$ and we prove, thanks to Aubin's theorem, the relative compactness of this interpolant in a weak space. We then deduce the compactness of $c$ in $L^{1}\left(0, T ; L_{\mathrm{loc}}^{1}(\Omega)\right)$.

Step 1: an affine interpolant of $c$.

We define $\widetilde{c}:[0, T[\times \Omega \rightarrow \mathbb{R}$ as the function continuous in time and affine on each time interval $[l k,(l+1) k[$, which is equal to $c^{l}$ at $t=l k$. Hence, for all $n=1, \ldots, N_{k}$ and all $t \in[(n-1) k, n k[$,

$$
\widetilde{c}(t, \cdot)=\frac{t-(n-1) k}{k} c_{K}^{n}+\frac{n k-t}{k} c_{K}^{n-1} \text { on } K .
$$

As $\widetilde{c}$ is, at each time, a mean value of values of $c$ and of $c^{0}=\left(c_{K}^{0}\right)_{K \in \mathcal{M}}$, the estimates of Proposition 3.2 and the definition of $\left(c_{K}^{0}\right)_{K \in \mathcal{M}}$ show that we have a bound on $\|\left.\widetilde{c}\right|_{L^{\infty}\left(0, T ; L^{2}(\Omega)\right)}$.

For all $n=1, \ldots, N_{k}$ and all $t \in\left[(n-1) k, n k\left[\right.\right.$, we have $\partial_{t} \widetilde{c}(t, \cdot)=\frac{c_{K}^{n}-c_{K}^{n-1}}{k}$ on $K$. Hence, denoting $\Phi_{\mathcal{D}}$ the function on $\Omega$ equal to $\Phi_{K}$ on $K$ and taking $\varphi \in C_{c}^{2}(\Omega)$, we deduce from (2.12) that, if $\varphi_{K}$ is the mean value of $\varphi$ on $K$,

$$
\begin{aligned}
\int_{\Omega} \Phi_{\mathcal{D}}(x) \partial_{t} \widetilde{c}(t, x) \varphi(x) d x & \\
= & \sum_{K \in \mathcal{M}} \mathrm{m}(K) \Phi_{K} \frac{c_{K}^{n}-c_{K}^{n-1}}{k} \varphi_{K} \\
= & \sum_{K \in \mathcal{M}} \sum_{\sigma \in \mathcal{E}_{K}} G_{K, \sigma}^{n} \varphi_{K}-\sum_{K \in \mathcal{M}} \sum_{\sigma=K \mid L \in \mathcal{E}_{K} \cap \mathcal{E}_{\text {int }}}\left[\left(-F_{K, \sigma}^{n}\right)^{+} c_{K}^{n}-\left(-F_{K, \sigma}^{n}\right)^{-} c_{L}^{n}\right] \varphi_{K} \\
& -\sum_{K \in \mathcal{M}} \mathrm{m}(K) q_{K}^{-, n} c_{K}^{n} \varphi_{K}+\sum_{K \in \mathcal{M}} \mathrm{m}(K) q_{K}^{+, n} \widehat{c}_{K}^{n} \varphi_{K} .
\end{aligned}
$$

Let us denote $T_{1}, T_{2}, T_{3}$ and $T_{4}$ the four terms of the right-hand side of this equality. In the following, $C_{i}$ denote constants which do not depend on $k, \mathcal{D}, n, K$ or $\varphi$; we induce $C_{c}^{2}(\Omega)$ with the norm $\|\varphi\|=$ $\sup _{x \in \Omega}\left(|\varphi(x)|+|\nabla \varphi(x)|+\left|D^{2} \varphi(x)\right|\right)$.

Since $\mathbf{x}_{K}$ is the barycenter of $K$ and $\varphi$ is regular, for all $\sigma \in \mathcal{E}_{K}$ we have

$$
\begin{aligned}
\varphi\left(\mathbf{x}_{\sigma}\right)-\varphi_{K}= & \frac{1}{\mathrm{~m}(K)} \int_{K}\left(\varphi\left(\mathbf{x}_{\sigma}\right)-\varphi(x)\right) d x \\
= & \frac{1}{\mathrm{~m}(K)} \int_{K} \nabla \varphi(x) \cdot\left(\mathbf{x}_{\sigma}-x\right) d x \\
& +\frac{1}{\mathrm{~m}(K)} \int_{K} \int_{0}^{1} D^{2} \varphi\left(x+t\left(\mathbf{x}_{\sigma}-x\right)\right)\left(\mathbf{x}_{\sigma}-x\right) \cdot\left(\mathbf{x}_{\sigma}-x\right) d x d t \\
= & \frac{1}{\mathrm{~m}(K)} \int_{K} \nabla \varphi\left(\mathbf{x}_{K}\right) \cdot\left(\mathbf{x}_{\sigma}-x\right) d x+R_{K, \sigma} \\
= & \nabla \varphi\left(\mathbf{x}_{K}\right) \cdot\left(\mathbf{x}_{\sigma}-\mathbf{x}_{K}\right)+R_{K, \sigma}
\end{aligned}
$$


with $\left|R_{K, \sigma}\right| \leq C_{7}|| \varphi|| \operatorname{diam}(K)^{2}$. Hence,

$$
\varphi_{L}-\varphi_{K}=\nabla \varphi\left(\mathbf{x}_{K}\right) \cdot\left(\mathbf{x}_{\sigma}-\mathbf{x}_{K}\right)+\nabla \varphi\left(\mathbf{x}_{L}\right) \cdot\left(\mathbf{x}_{L}-\mathbf{x}_{\sigma}\right)+R_{K, \sigma}-R_{L, \sigma} .
$$

By (2.10), (2.13) and (2.11), we therefore write

$$
\begin{aligned}
-T_{1}= & \sum_{\sigma=K \mid L \in \mathcal{E}_{\text {int }}} G_{K, \sigma}^{n}\left(\varphi_{L}-\varphi_{K}\right) \\
= & \sum_{\sigma=K \mid L \in \mathcal{E}_{\text {int }}} G_{K, \sigma}^{n}\left(\nabla \varphi\left(\mathbf{x}_{K}\right) \cdot\left(\mathbf{x}_{\sigma}-\mathbf{x}_{K}\right)+\nabla \varphi\left(\mathbf{x}_{L}\right) \cdot\left(\mathbf{x}_{L}-\mathbf{x}_{\sigma}\right)\right) \\
& +\sum_{\sigma=K \mid L \in \mathcal{E}_{\text {int }}} G_{K, \sigma}^{n}\left(R_{K, \sigma}-R_{L, \sigma}\right) \\
= & \sum_{K \in \mathcal{M}} \nabla \varphi\left(\mathbf{x}_{K}\right) \cdot \sum_{\sigma \in \mathcal{E}_{K}} G_{K, \sigma}^{n}\left(\mathbf{x}_{\sigma}-\mathbf{x}_{K}\right)+\sum_{K \in \mathcal{M}} \sum_{\sigma \in \mathcal{E}_{K}} G_{K, \sigma}^{n} R_{K, \sigma} \\
= & \sum_{K \in \mathcal{M}} \mathrm{m}(K) \nabla \varphi\left(\mathbf{x}_{K}\right) \cdot D_{K}\left(\mathbf{U}_{K}^{n}\right) \mathbf{w}_{K}^{n}+\sum_{K \in \mathcal{M}} \sum_{\sigma \in \mathcal{E}_{K}} G_{K, \sigma}^{n} R_{K, \sigma} .
\end{aligned}
$$

On one hand, thanks to (1.11) and to the estimate on $\mathbf{U}$ in $L^{\infty}\left(0, T ; L^{2}(\Omega)\right)^{d}$ (which gives in particular an estimate in $\left.L^{\infty}\left(0, T ; L^{1}(\Omega)\right)^{d}\right)$, we have

$$
\begin{aligned}
\left|\sum_{K \in \mathcal{M}} \mathrm{m}(K) \nabla \varphi\left(\mathbf{x}_{K}\right) \cdot D_{K}\left(\mathbf{U}_{K}^{n}\right) \mathbf{w}_{K}^{n}\right| & \leq C_{8}\|\varphi\| \sum_{K \in \mathcal{M}} \mathrm{m}(K)\left(1+\left|\mathbf{U}_{K}^{n}\right|\right)\left|\mathbf{w}_{K}^{n}\right| \\
& \leq C_{9}\|\varphi\|\left(\sum_{K \in \mathcal{M}} \mathrm{m}(K)\left(1+\left|\mathbf{U}_{K}^{n}\right|\right)\left|\mathbf{w}_{K}^{n}\right|^{2}\right)^{\frac{1}{2}} .
\end{aligned}
$$

On the other hand,

$$
\begin{aligned}
& \left|\sum_{K \in \mathcal{M}} \sum_{\sigma \in \mathcal{E}_{K}} G_{K, \sigma}^{n} R_{K, \sigma}\right| \\
& \quad \leq C_{7}\|\varphi\| \sum_{K \in \mathcal{M}} \sum_{\sigma \in \mathcal{E}_{K}} \operatorname{diam}(K)^{2}\left|G_{K, \sigma}^{n}\right| \\
& \quad \leq C_{7}\|\varphi\|\left(\sum_{K \in \mathcal{M}} \sum_{\sigma \in \mathcal{E}_{K}} \nu_{K} \mathrm{~m}(K)\left|G_{K, \sigma}^{n}\right|^{2}\right)^{\frac{1}{2}}\left(\sum_{K \in \mathcal{M}} \sum_{\sigma \in \mathcal{E}_{K}} \frac{\operatorname{diam}(K)^{4}}{\nu_{K} \mathrm{~m}(K)}\right)^{\frac{1}{2}} \\
& \quad \leq C_{10}\|\varphi\|\left(\sum_{K \in \mathcal{M}} \sum_{\sigma \in \mathcal{E}_{K}} \nu_{K} \mathrm{~m}(K)\left|G_{K, \sigma}^{n}\right|^{2}\right)^{\frac{1}{2}}\left(\sum_{K \in \mathcal{M}} \operatorname{diam}(K)^{4-2 d-\beta} \mathrm{m}(K)\right)^{\frac{1}{2}}
\end{aligned}
$$

because (2.1) and the definition of $\nu_{K}$ imply

$$
\frac{\operatorname{diam}(K)^{4}}{\nu_{K} \mathrm{~m}(K)}=\mathrm{m}(K) \frac{\operatorname{diam}(K)^{4-\beta}}{\nu_{0} \mathrm{~m}(K)^{2}} \leq \frac{1}{\nu_{0}}\left(\frac{\operatorname{regul}(\mathcal{D})}{\omega_{d}}\right)^{2} \mathrm{~m}(K) \operatorname{diam}(K)^{4-2 d-\beta} .
$$

But $4-2 d-\beta \geq 0$ and thus $\operatorname{diam}(K)^{4-2 d-\beta} \leq \operatorname{diam}(\Omega)^{4-2 d-\beta}$. Using this in (5.5) and injecting the result along with (5.4) in (5.3), we deduce the final estimate on $T_{1}$ :

$$
\left|T_{1}\right| \leq C_{11}\|\varphi\|\left(\sum_{K \in \mathcal{M}} \mathrm{m}(K)\left(1+\left|\mathbf{U}_{K}^{n}\right|\right)\left|\mathbf{w}_{K}^{n}\right|^{2}\right)^{\frac{1}{2}}+C_{11}\|\varphi\|\left(\sum_{K \in \mathcal{M}} \sum_{\sigma \in \mathcal{E}_{K}} \nu_{K} \mathrm{~m}(K)\left|G_{K, \sigma}^{n}\right|^{2}\right)^{\frac{1}{2}} .
$$


For $\sigma=K \mid L$, we let $b_{K, \sigma}^{n}=\left(-F_{K, \sigma}^{n}\right)^{+} c_{K}^{n}-\left(-F_{K, \sigma}^{n}\right)^{-} c_{L}^{n}$ and, by $(2.3)$, we have $b_{K, \sigma}^{n}=-b_{L, \sigma}^{n}$. Hence, by $(5.2)$

$$
\begin{aligned}
T_{2} & =\sum_{\sigma=K \mid L \in \mathcal{E}_{\mathrm{int}}} b_{K, \sigma}^{n}\left(\varphi_{L}-\varphi_{K}\right) \\
& =\sum_{\sigma=K \mid L \in \mathcal{E}_{\mathrm{int}}} b_{K, \sigma}^{n}\left(\left(\nabla \varphi\left(\mathbf{x}_{K}\right) \cdot\left(\mathbf{x}_{\sigma}-\mathbf{x}_{K}\right)+\nabla \varphi\left(\mathbf{x}_{L}\right) \cdot\left(\mathbf{x}_{L}-\mathbf{x}_{\sigma}\right)\right)+\sum_{\sigma=K \mid L \in \mathcal{E}_{\mathrm{int}}} b_{K, \sigma}^{n}\left(R_{K, \sigma}-R_{L, \sigma}\right)\right. \\
& =\sum_{K \in \mathcal{M}} \nabla \varphi\left(\mathbf{x}_{K}\right) \cdot \sum_{\sigma \in \mathcal{E}_{K} \cap \mathcal{E}_{\mathrm{int}}} b_{K, \sigma}^{n}\left(\mathbf{x}_{\sigma}-\mathbf{x}_{K}\right)+\sum_{K \in \mathcal{M}} \sum_{\sigma \in \mathcal{E}_{K} \cap \mathcal{E}_{\mathrm{int}}} b_{K, \sigma}^{n} R_{K, \sigma}
\end{aligned}
$$

But $b_{K, \sigma}^{n}=-F_{K, \sigma}^{n} c_{K}^{n}+\left(-F_{K, \sigma}^{n}\right)^{-}\left(c_{K}^{n}-c_{L}^{n}\right)$ and thus, by (2.8) and (2.5),

$$
T_{2}=-\sum_{K \in \mathcal{M}} c_{K}^{n} \nabla \varphi\left(\mathbf{x}_{K}\right) \cdot \sum_{\sigma \in \mathcal{E}_{K}} F_{K, \sigma}^{n}\left(\mathbf{x}_{\sigma}-\mathbf{x}_{K}\right)+T_{5}=\sum_{K \in \mathcal{M}} \mathrm{m}(K) c_{K}^{n} \nabla \varphi\left(\mathbf{x}_{K}\right) \cdot \mathbf{U}_{K}^{n}+T_{5}
$$

with

$$
T_{5}=\sum_{K \in \mathcal{M}} \nabla \varphi\left(\mathbf{x}_{K}\right) \cdot \sum_{\sigma=K \mid L \in \mathcal{E}_{K} \cap \mathcal{E}_{\text {int }}}\left(-F_{K, \sigma}^{n}\right)^{-}\left(c_{K}^{n}-c_{L}^{n}\right)\left(\mathbf{x}_{\sigma}-\mathbf{x}_{K}\right)+\sum_{K \in \mathcal{M}} \sum_{\sigma \in \mathcal{E}_{K} \cap \mathcal{E}_{\text {int }}} b_{K, \sigma}^{n} R_{K, \sigma} .
$$

Let us first estimate $T_{5}$. The corresponding calculus will be later useful in the proof of the convergence of the concentration. We have:

$$
\left|T_{5}\right| \leq\|\varphi\| \sum_{K \in \mathcal{M}} \sum_{\sigma=K \mid L \in \mathcal{E}_{K} \cap \mathcal{E}_{\text {int }}}\left|F_{K, \sigma}^{n}\right|\left|c_{K}^{n}-c_{L}^{n}\right| \operatorname{diam}(K)+C_{7}|| \varphi \| \sum_{K \in \mathcal{M}} \sum_{\sigma \in \mathcal{E}_{K} \cap \mathcal{E}_{\text {int }}}\left|b_{K, \sigma}^{n}\right| \operatorname{diam}(K)^{2} .
$$

But (2.9) entails

$$
\left|c_{K}^{n}-c_{L}^{n}\right| \leq\left|\mathbf{w}_{K}^{n}\right| \operatorname{diam}(K)+\left|\mathbf{w}_{L}^{n}\right| \operatorname{diam}(L)+\nu_{K} \mathrm{~m}(K)\left|G_{K, \sigma}^{n}\right|+\nu_{L} \mathrm{~m}(L)\left|G_{L, \sigma}^{n}\right|
$$

and thus, using $\left|F_{K, \sigma}^{n}\right|=\left|F_{L, \sigma}^{n}\right|$ whenever $\sigma=K \mid L$,

$$
\begin{aligned}
\left|b_{K, \sigma}^{n}\right| \leq & \left|F_{K, \sigma}^{n}\right|\left|c_{K}^{n}\right|+\left|F_{K, \sigma}^{n}\right|\left|\mathbf{w}_{K}^{n}\right| \operatorname{diam}(K)+\left|F_{K, \sigma}^{n}\right|\left|\mathbf{w}_{L}^{n}\right| \operatorname{diam}(L)+\nu_{K} \mathrm{~m}(K)\left|F_{K, \sigma}^{n}\right|\left|G_{K, \sigma}^{n}\right| \\
& +\nu_{L} \mathrm{~m}(L)\left|F_{L, \sigma}^{n}\right|\left|G_{L, \sigma}^{n}\right| .
\end{aligned}
$$

Injecting these two estimates in $(5.8)$ and bounding $\operatorname{diam}(K)$ either by $\operatorname{diam}(\Omega)$ or by $\operatorname{size}(\mathcal{D})$ leads to

$$
\begin{aligned}
\left|T_{5}\right| \leq & C_{12}|| \varphi \| \sum_{K \in \mathcal{M}} \sum_{\sigma \in \mathcal{E}_{K}}\left|F_{K, \sigma}^{n}\right|\left(\left|\mathbf{w}_{K}^{n}\right|+\left|c_{K}^{n}\right|\right) \operatorname{diam}(K)^{2} \\
& +C_{12}\|\varphi\| \sum_{K \in \mathcal{M}} \sum_{\sigma=K \mid L \in \mathcal{E}_{K} \cap \mathcal{E}_{\text {int }}}\left|F_{K, \sigma}^{n}\right|\left|\mathbf{w}_{L}^{n}\right| \operatorname{diam}(K) \operatorname{diam}(L) \\
& +C_{12}\|\varphi\| \operatorname{size}(\mathcal{D}) \sum_{K \in \mathcal{M}} \sum_{\sigma \in \mathcal{E}_{K}} \nu_{K} \mathrm{~m}(K)\left|F_{K, \sigma}^{n}\right|\left|G_{K, \sigma}^{n}\right| \\
& +C_{12}\|\varphi\| \operatorname{size}(\mathcal{D}) \sum_{K \in \mathcal{M}} \sum_{\sigma=K \mid L \in \mathcal{E}_{K} \cap \mathcal{E}_{\mathrm{int}}} \nu_{L} \mathrm{~m}(L)\left|F_{L, \sigma}^{n}\right|\left|G_{L, \sigma}^{n}\right| .
\end{aligned}
$$

We successively apply Cauchy-Schwarz inequality, the fact that $\operatorname{regul}(\mathcal{D})$ is bounded, the inequality $(5.6)$ and the estimates on $F$ from Proposition 3.1. It yields:

$$
\begin{aligned}
& \sum_{K \in \mathcal{M}} \sum_{\sigma \in \mathcal{E}_{K}}\left|F_{K, \sigma}^{n}\right|\left(\left|\mathbf{w}_{K}^{n}\right|+\left|c_{K}^{n}\right|\right) \operatorname{diam}(K)^{2} \\
& \leq C_{13}\left(\sum_{K \in \mathcal{M}} \sum_{\sigma \in \mathcal{E}_{K}} \nu_{K} \mathrm{~m}(K)\left|F_{K, \sigma}^{n}\right|^{2}\right)^{\frac{1}{2}}\left(\sum_{K \in \mathcal{M}} \mathrm{m}(K) \operatorname{diam}(K)^{4-2 d-\beta}\left(\left|\mathbf{w}_{K}^{n}\right|+\left|c_{K}^{n}\right|\right)^{2}\right)^{\frac{1}{2}} \\
& \leq C_{14} \operatorname{size}(\mathcal{D})^{\frac{4-2 d-\beta}{2}}\left(\sum_{K \in \mathcal{M}} \mathrm{m}(K)\left(\left|\mathbf{w}_{K}^{n}\right|+\left|c_{K}^{n}\right|\right)^{2}\right)^{\frac{1}{2}}
\end{aligned}
$$


Then, we note that (thanks to (2.8))

$$
\sum_{K \in \mathcal{M}} \sum_{\sigma=K \mid L \in \mathcal{E}_{K} \cap \mathcal{E}_{\text {int }}} \nu_{L} \mathrm{~m}(L)\left|F_{L, \sigma}^{n}\right|\left|G_{L, \sigma}^{n}\right|=\sum_{K \in \mathcal{M}} \sum_{\sigma \in \mathcal{E}_{K}} \nu_{K} \mathrm{~m}(K)\left|F_{K, \sigma}^{n}\right|\left|G_{K, \sigma}^{n}\right|
$$

and, using the estimates on $F$ from Proposition 3.1, we get

$$
\sum_{K \in \mathcal{M}} \sum_{\sigma \in \mathcal{E}_{K}} \nu_{K} \mathrm{~m}(K)\left|F_{K, \sigma}^{n}\right|\left|G_{K, \sigma}^{n}\right| \leq C_{15}\left(\sum_{K \in \mathcal{M}} \sum_{\sigma \in \mathcal{E}_{K}} \nu_{K} \mathrm{~m}(K)\left|G_{K, \sigma}^{n}\right|^{2}\right)^{\frac{1}{2}} .
$$

Using the fact that $\nu_{K}=\nu_{0} \operatorname{diam}(K)^{\beta}$ and inequalities (2.1) and (2.14), we get

$$
\begin{aligned}
& \sum_{K \in \mathcal{M}} \sum_{\sigma=K \mid L \in \mathcal{E}_{K} \cap \mathcal{E}_{\text {int }}}\left|F_{K, \sigma}^{n}\right|\left|\mathbf{w}_{L}^{n}\right| \operatorname{diam}(K) \operatorname{diam}(L) \\
& \leq\left(\sum_{K \in \mathcal{M}} \sum_{\sigma \in \mathcal{E}_{K}} \nu_{K} \mathrm{~m}(K)\left|F_{K, \sigma}^{n}\right|^{2}\right)^{\frac{1}{2}}\left(\sum_{K \in \mathcal{M}} \sum_{\sigma=K \mid L \in \mathcal{E}_{K} \cap \mathcal{E}_{\text {int }}} \frac{\operatorname{diam}(K)^{2} \operatorname{diam}(L)^{2}}{\nu_{K} \mathrm{~m}(K)}\left|\mathbf{w}_{L}^{n}\right|^{2}\right)^{\frac{1}{2}} \\
& \leq C_{16}\left(\sum_{K \in \mathcal{M}} \sum_{\sigma \in \mathcal{E}_{K}} \nu_{K} \mathrm{~m}(K)\left|F_{K, \sigma}^{n}\right|^{2}\right)^{\frac{1}{2}}\left(\sum_{L \in \mathcal{M}}\left|\mathbf{w}_{L}^{n}\right|^{2} \sum_{\sigma=L \mid K \in \mathcal{E}_{L} \cap \mathcal{E}_{\text {int }}} \operatorname{diam}(K)^{2-\beta-d} \operatorname{diam}(L)^{2}\right)^{\frac{1}{2}} \\
& \leq C_{17}\left(\sum_{K \in \mathcal{M}} \sum_{\sigma \in \mathcal{E}_{K}} \nu_{K} \mathrm{~m}(K)\left|F_{K, \sigma}^{n}\right|^{2}\right)^{\frac{1}{2}}\left(\sum_{L \in \mathcal{M}} \mathrm{m}(L)\left|\mathbf{w}_{L}^{n}\right|^{2}\right)^{\frac{1}{2}} .
\end{aligned}
$$

Finally, gathering (5.9), (5.10), (5.11), (5.12) and (5.13), it yields

$$
\begin{aligned}
&\left|T_{2}-\sum_{K \in \mathcal{M}} \mathrm{m}(K) c_{K}^{n} \nabla \varphi\left(\mathbf{x}_{K}\right) \cdot \mathbf{U}_{K}^{n}\right| \\
&=\mid\left|T_{5}\right| \\
& \leq C_{18}\|\varphi\| \operatorname{size}(\mathcal{D})^{\frac{4-2 d-\beta}{2}}\left(\sum_{K \in \mathcal{M}} \mathrm{m}(K)\left(\left|\mathbf{w}_{K}^{n}\right|+\left|c_{K}^{n}\right|\right)^{2}\right)^{\frac{1}{2}} \\
&+C_{18}\|\varphi\|\left(\sum_{K \in \mathcal{M}} \sum_{\sigma \in \mathcal{E}_{K}} \nu_{K} \mathrm{~m}(K)\left|F_{K, \sigma}^{n}\right|^{2}\right)^{\frac{1}{2}}\left(\sum_{K \in \mathcal{M}} \mathrm{m}(K)\left|\mathbf{w}_{K}^{n}\right|^{2}\right)^{\frac{1}{2}} \\
&+C_{18}\|\varphi\| \operatorname{size}(\mathcal{D})\left(\sum_{K \in \mathcal{M}} \sum_{\sigma \in \mathcal{E}_{K}} \nu_{K} \mathrm{~m}(K)\left|G_{K, \sigma}^{n}\right|^{2}\right)^{\frac{1}{2}} .
\end{aligned}
$$

Thanks to the $L^{\infty}\left(0, T ; L^{2}(\Omega)\right)$ estimates on $c$ and $\mathbf{U}$, we also have

$$
\begin{aligned}
\left|\sum_{K \in \mathcal{M}} \mathrm{m}(K) c_{K}^{n} \nabla \varphi\left(\mathbf{x}_{K}\right) \cdot \mathbf{U}_{K}^{n}\right| & \leq\|\varphi\|\left(\sum_{K \in \mathcal{M}} \mathrm{m}(K)\left|c_{K}^{n}\right|^{2}\right)^{\frac{1}{2}}\left(\sum_{K \in \mathcal{M}} \mathrm{m}(K)\left|\mathbf{U}_{K}^{n}\right|^{2}\right)^{\frac{1}{2}} \\
& \leq C_{19}\|\varphi\|
\end{aligned}
$$

and, using the bound on the fluxes $F_{K, \sigma}^{n}$ from Proposition 3.1, the final estimate on $T_{2}$ reads

$$
\begin{aligned}
\left|T_{2}\right| \leq & C_{20}\|\varphi\|+C_{20}\|\varphi\|\left(\sum_{K \in \mathcal{M}} \mathrm{m}(K)\left(\left|\mathbf{w}_{K}^{n}\right|+\left|c_{K}^{n}\right|\right)^{2}\right)^{\frac{1}{2}} \\
& +C_{20}\|\varphi\|\left(\sum_{K \in \mathcal{M}} \sum_{\sigma \in \mathcal{E}_{K}} \nu_{K} \mathrm{~m}(K)\left|G_{K, \sigma}^{n}\right|^{2}\right)^{\frac{1}{2}} .
\end{aligned}
$$


The estimates on $T_{3}$ and $T_{4}$ are straightforward, thanks to the bound on $c$ in $L^{\infty}\left(0, T ; L^{2}(\Omega)\right)$; injecting (5.7) and (5.15) in (5.1), we obtain, for all $n=1, \ldots, N_{k}$ and all $t \in[(n-1) k, n k[$,

$$
\begin{aligned}
& \left|\int_{\Omega} \Phi_{\mathcal{D}}(x) \partial_{t} \widetilde{c}(t, x) \varphi(x) d x\right| \\
& \leq \quad C_{21}\|\varphi\|\left(\sum_{K \in \mathcal{M}} \mathrm{m}(K)\left(1+\left|\mathbf{U}_{K}^{n}\right|\right)\left|\mathbf{w}_{K}^{n}\right|^{2}\right)^{\frac{1}{2}}+C_{21}|| \varphi \|\left(\sum_{K \in \mathcal{M}} \sum_{\sigma \in \mathcal{E}_{K}} \nu_{K} \mathrm{~m}(K)\left|G_{K, \sigma}^{n}\right|^{2}\right)^{\frac{1}{2}} \\
& \quad+C_{21}|| \varphi\left\|+C_{21}\right\| \varphi \|\left(\sum_{K \in \mathcal{M}} \mathrm{m}(K)\left(\left|\mathbf{w}_{K}^{n}\right|+\left|c_{K}^{n}\right|\right)^{2}\right)^{\frac{1}{2}} \cdot
\end{aligned}
$$

Since this inequality is satisfied for all $\varphi \in C_{c}^{2}(\Omega)$, and since $\Phi_{\mathcal{D}}$ does not depend on $t$, this gives an estimate on $\left\|\partial_{t}\left(\Phi_{\mathcal{D}} \widetilde{c}\right)(t, \cdot)\right\|_{\left(C_{c}^{2}(\Omega)\right)^{\prime}}$ which, squared, leads to

$$
\begin{aligned}
\left\|\partial_{t}\left(\Phi_{\mathcal{D}} \widetilde{c}\right)(t, \cdot)\right\|_{\left(C_{c}^{2}(\Omega)\right)^{\prime}}^{2} \leq & C_{22} \sum_{K \in \mathcal{M}} \mathrm{m}(K)\left(1+\left|\mathbf{U}_{K}^{n}\right|\right)\left|\mathbf{w}_{K}^{n}\right|^{2}+C_{22} \sum_{K \in \mathcal{M}} \sum_{\sigma \in \mathcal{E}_{K}} \nu_{K} \mathrm{~m}(K)\left|G_{K, \sigma}^{n}\right|^{2} \\
& +C_{22}+C_{22} \sum_{K \in \mathcal{M}} \mathrm{m}(K)\left(\left|\mathbf{w}_{K}^{n}\right|+\left|c_{K}^{n}\right|\right)^{2}
\end{aligned}
$$

for all $n=1, \ldots, N_{k}$ and all $t \in[(n-1) k, n k[$. Integrating this last inequality on $t \in[(n-1) k, n k[$ (which, as far as the right-hand side is concerned, comes down to multiplying by $k$ since it does not depend on $t$ ) and summing on $n=1, \ldots, N_{k}$, we prove, thanks to the estimates of Proposition 3.2, that $\partial_{t}\left(\Phi_{\mathcal{D}} \widetilde{c}\right)$ is bounded in $L^{2}\left(0, T ;\left(C_{c}^{2}(\Omega)\right)^{\prime}\right)$.

Noting that $\Phi_{\mathcal{D}} \widetilde{c}$ is bounded in $L^{\infty}\left(0, T ; L^{2}(\Omega)\right)$ (because $\widetilde{c}$ is bounded in this space and $\Phi_{\mathcal{D}}$ is bounded in $L^{\infty}(\Omega)$ ), and since $L^{2}(\Omega)$ is continuously embedded in $\left(C_{c}^{2}(\Omega)\right)^{\prime}$ (via the natural embedding $f \rightarrow(g \rightarrow$ $\left.\int_{\Omega} f g\right)$ ), this shows that $\Phi_{\mathcal{D}} \widetilde{c}$ is bounded in $H^{1}\left(0, T ;\left(C_{c}^{2}(\Omega)\right)^{\prime}\right)$. But $C_{c}^{2}(\Omega)$ is compactly and densely embedded in $C_{0}(\Omega)$, and, by duality, $\left(C_{0}(\Omega)\right)^{\prime}$ (the space of bounded measures on $\Omega$ ) is compactly embedded in $\left(C_{c}^{2}(\Omega)\right)^{\prime}$. Since $L^{2}(\Omega)$ is continuously embedded in $\left(C_{0}(\Omega)\right)^{\prime}$ (via an embedding which is compatible with the preceding one), the embedding of $L^{2}(\Omega)$ in $\left(C_{c}^{2}(\Omega)\right)^{\prime}$ is in fact compact. Hence, by Aubin's compactness theorem we deduce that $\Phi_{\mathcal{D}} \widetilde{c}$ is relatively compact in $C\left([0, T] ;\left(C_{c}^{2}(\Omega)\right)^{\prime}\right)$.

Step 2: conclusion.

For all $n=1, \ldots, N_{k}$ and $t \in\left[(n-1) k, n k\left[\right.\right.$, we have $\Phi_{\mathcal{D}} c(t, \cdot)=\Phi_{\mathcal{D}} \widetilde{c}(n k, \cdot)$ on $\Omega$ (these functions are both equal to $\Phi_{K} c_{K}^{n}$ on each $\left.K \in \mathcal{M}\right)$. We also know, see e.g. [7], that $H^{1}\left(0, T ;\left(C_{c}^{2}(\Omega)\right)^{\prime}\right)$ is continuously embedded in $C^{1 / 2}\left([0, T] ;\left(C_{c}^{2}(\Omega)\right)^{\prime}\right)$ (the space of $1 / 2$-hölder continuous functions $\left.[0, T] \rightarrow\left(C_{c}^{2}(\Omega)\right)^{\prime}\right)$. Hence, $\Phi_{\mathcal{D}} \widetilde{c}$ is also bounded in $C^{1 / 2}\left([0, T] ;\left(C_{c}^{2}(\Omega)\right)^{\prime}\right)$ and there exists $C_{23}$ not depending on $k$ or $\mathcal{D}$ such that, for all $n=1, \ldots, N_{k}$ and all $t \in[(n-1) k, n k[$,

$$
\left\|\Phi_{\mathcal{D}} c(t, \cdot)-\Phi_{\mathcal{D}} \widetilde{c}(t, \cdot)\right\|_{\left(C_{c}^{2}(\Omega)\right)^{\prime}}=\left\|\Phi_{\mathcal{D}} \widetilde{c}(n k, \cdot)-\Phi_{\mathcal{D}} \widetilde{c}(t, \cdot)\right\|_{\left(C_{c}^{2}(\Omega)\right)^{\prime}} \leq C_{23} \sqrt{k} .
$$

This means that, as $k \rightarrow 0, \Phi_{\mathcal{D}} c-\Phi_{\mathcal{D}} \widetilde{c} \rightarrow 0$ in $L^{\infty}\left(0, T ;\left(C_{c}^{2}(\Omega)\right)^{\prime}\right)$; since $\Phi_{\mathcal{D}} \widetilde{c}$ is relatively compact in this space, we deduce that $\Phi_{\mathcal{D}} c$ is also relatively compact in this same space, and thus in particular in $L^{1}\left(0, T ;\left(C_{c}^{2}(\Omega)\right)^{\prime}\right)$.

Let $n=1, \ldots, N_{k}$ and $t \in[(n-1) k, n k[$. By $(2.9)$, Lemma 7.3 gives, for all $\omega$ relatively compact in $\Omega$ and all $|\xi|<\operatorname{dist}\left(\omega, \mathbb{R}^{d} \backslash \Omega\right)$,

$$
\| c(t, \cdot+\xi)-\left.c(t, \cdot)\right|_{L^{1}(\omega)} \leq C_{24}|\xi| \sum_{K \in \mathcal{M}} \mathrm{m}(K)\left|\mathbf{w}_{K}^{n}\right|+C_{24}|\xi| \sum_{K \in \mathcal{M}} \sum_{\sigma \in \mathcal{E}_{K}} \operatorname{diam}(K)^{d-1} \nu_{K} \mathrm{~m}(K)\left|G_{K, \sigma}^{n}\right| .
$$

Integrating on $t \in\left[(n-1) k, n k\left[\right.\right.$ and summing on $n=1, \ldots, N_{k}$, this implies

$$
\|c(\cdot, \cdot+\xi)-c\|_{L^{1}(] 0, T[\times \omega)}
$$




$$
\begin{aligned}
\leq & C_{24}|\xi| \sum_{n=1}^{N_{k}} k \sum_{K \in \mathcal{M}} \mathrm{m}(K)\left|\mathbf{w}_{K}^{n}\right|+C_{24}|\xi| \sum_{n=1}^{N_{k}} k \sum_{K \in \mathcal{M}} \sum_{\sigma \in \mathcal{E}_{K}} \operatorname{diam}(K)^{d-1} \nu_{K} \mathrm{~m}(K)\left|G_{K, \sigma}^{n}\right| \\
\leq & C_{24}|\xi| \| \mathbf{w} \mid L_{L^{1}(] 0, T[\times \Omega)^{d}} \\
& +C_{24}|\xi|\left(\sum_{n=1}^{N_{k}} k \sum_{K \in \mathcal{M}} \sum_{\sigma \in \mathcal{E}_{K}} \operatorname{diam}(K)^{2 d-2} \nu_{K} \mathrm{~m}(K)\right)^{\frac{1}{2}}\left(\sum_{n=1}^{N_{k}} k \sum_{K \in \mathcal{M}} \sum_{\sigma \in \mathcal{E}_{K}} \nu_{K} \mathrm{~m}(K)\left|G_{K, \sigma}^{n}\right|^{2}\right)^{\frac{1}{2}} \\
\leq & C_{25}|\xi|+C_{25}|\xi|\left(\sum_{n=1}^{N_{k}} k \sum_{K \in \mathcal{M}} \operatorname{diam}(K)^{2 d-2+\beta} \mathrm{m}(K)\right)^{\frac{1}{2}}
\end{aligned}
$$

thanks to the estimates of Proposition 3.2. But $2 d-2+\beta \geq 0$ and thus $\operatorname{diam}(K)^{2 d-2+\beta} \leq \operatorname{diam}(\Omega)^{2 d-2+\beta}$. Hence, we see that

$$
\|c(\cdot, \cdot+\xi)-c\|_{L^{1}(] 0, T[\times \omega)} \rightarrow 0 \quad \text { as } \xi \rightarrow 0, \text { independently of } k \text { or } \mathcal{D} .
$$

Since $\Phi_{\mathcal{D}}$ is bounded in $L^{\infty}(\Omega)$ and $c$ is bounded in $L^{\infty}\left(0, T ; L^{2}(\Omega)\right)$, we have

$$
\begin{aligned}
\|\left(\Phi_{\mathcal{D}} c\right)(\cdot, \cdot+\xi)-\Phi_{\mathcal{D}} c & \|_{L^{1}(] 0, T[\times \omega)} \\
& =\left\|\Phi_{\mathcal{D}}(\cdot+\xi)(c(\cdot, \cdot+\xi)-c)+\left(\Phi_{\mathcal{D}}(\cdot+\xi)-\Phi_{\mathcal{D}}\right) c\right\|_{L^{1}(] 0, T[\times \omega)} \\
& \leq C_{26}\|c(\cdot, \cdot+\xi)-c\|_{L^{1}(] 0, T[\times \omega)}+\left\|\Phi_{\mathcal{D}}(\cdot+\xi)-\Phi_{\mathcal{D}}\right\|_{L^{2}(\omega)}\|c\|_{L^{1}\left(0, T ; L^{2}(\omega)\right)} \\
& \leq C_{26}\|c(\cdot, \cdot+\xi)-c\|_{L^{1}(] 0, T[\times \omega)}+C_{27}\left\|\Phi_{\mathcal{D}}(\cdot+\xi)-\Phi_{\mathcal{D}}\right\|_{L^{2}(\omega)}
\end{aligned}
$$

where $C_{26}$ and $C_{27}$ do not depend on $\mathcal{D}$ nor $k$. But it is classical that $\Phi_{\mathcal{D}} \rightarrow \Phi$ in $L^{2}(\Omega)$ as $\operatorname{size}(\mathcal{D}) \rightarrow 0$ $\left({ }^{3}\right)$, and thus $\left\|\Phi_{\mathcal{D}}(\cdot+\xi)-\Phi_{\mathcal{D}}\right\|_{L^{2}(\omega)} \rightarrow 0$ as $\xi \rightarrow 0$, independently of $\mathcal{D}$. Thanks to (5.16), we therefore obtain $\left\|\Phi_{\mathcal{D}} c(\cdot, \cdot+\xi)-\Phi_{\mathcal{D}} c\right\|_{L^{1}([0, T[\times \omega)} \rightarrow 0$ as $\xi \rightarrow 0$, independently of $k$ or $\mathcal{D}$. Since $\Phi_{\mathcal{D}} c$ is relatively compact in $L^{1}\left(0, T ;\left(C_{c}^{2}(\Omega)\right)^{\prime}\right)$, Lemma 7.5 then shows that $\Phi_{\mathcal{D}} c$ is relatively compact in $L^{1}\left(0, T ; L_{\text {loc }}^{1}(\Omega)\right)$. Up to a subsequence as $k \rightarrow 0$ and $\operatorname{size}(\mathcal{D}) \rightarrow 0$, we have $\Phi_{\mathcal{D}} c \rightarrow f$ in $L^{1}\left(0, T ; L_{\text {loc }}^{1}(\Omega)\right)$. Using again the fact that $\Phi_{\mathcal{D}} \rightarrow \Phi$ in $L^{2}(\Omega)$ we also have, up to another subsequence, $\Phi_{\mathcal{D}} \rightarrow \Phi$ a.e. on $\Omega$; moreover, $\Phi_{\mathcal{D}} \geq \Phi_{*}>0$ and thus $\frac{1}{\Phi_{\mathcal{D}}}$ stays bounded on $\Omega$ (independently of $\mathcal{D}$ ) and converges a.e. to $\frac{1}{\Phi}$. The dominated convergence theorem then shows that $c=\frac{1}{\Phi_{\mathcal{D}}} \Phi_{\mathcal{D}} c \rightarrow \frac{1}{\Phi} f$ in $L^{1}\left(0, T ; L_{\text {loc }}^{1}(\Omega)\right)$, which concludes the proof.

Remark 5.1 The usual way to obtain compactness on the solution to a Finite Volume discretization of a parabolic equation is to estimate the norm in $L^{2}(] 0, T[\times \Omega)$ of translations in time of the solution; basically, such estimates are possible because there is an estimate in $L^{2}\left(0, T ; H^{1}(\Omega)^{\prime}\right)$ on the time derivative of the continuous solution. However, as it can easily be seen on (1.8), the expected estimate on $\partial_{t} \bar{c}$ is in $L^{2}\left(0, T ; W^{1,4}(\Omega)^{\prime}\right)$ (because $\overline{\mathbf{U}} \in L^{\infty}\left(0, T ; L^{2}(\Omega)\right)^{d}$ and $\left.|\overline{\mathbf{U}}|^{1 / 2} \nabla \bar{c} \in L^{2}(] 0, T[\times \Omega)^{d}\right)$. Hence, we have little hope mimicking, in our situation, the technique that allows to pass from the continuous estimate on the time derivative in $L^{2}\left(0, T ; H^{1}(\Omega)^{\prime}\right)$ to the discrete translation estimate in $L^{2}(] 0, T[\times \Omega)$; in other words, if we search for a translation estimate, it must be in weaker spaces than $L^{2}(] 0, T[\times \Omega)$ (for example $L^{1}(] 0, T[\times \Omega)$ ), which requires manipulations that do not seem feasible in the discrete setting.

In the following, we extract a sequence such that $c$ converges in $L^{1}\left(0, T ; L_{\text {loc }}^{1}(\Omega)\right)$ to some $\bar{c}$.

\subsection{Convergence of the pressure}

We now turn to the convergence of $(p, \mathbf{v}, \mathbf{U})$. By Proposition 3.1, we can assume, up to a subsequence, that $p \rightarrow \bar{p}$ weakly-* in $L^{\infty}\left(0, T ; L^{2}(\Omega)\right.$ ) (which is the dual space of $L^{1}\left(0, T ; L^{2}(\Omega)\right)$ ) and that $\mathbf{v} \rightarrow \overline{\mathbf{v}}$ weakly-* in $L^{\infty}\left(0, T ; L^{2}(\Omega)\right)^{d}$. Since $\int_{\Omega} p(t, \cdot)=0$ for all $\left.t \in\right] 0, T\left[\right.$, it is quite clear that $\int_{\Omega} \bar{p}(t, \cdot)=0$ for

\footnotetext{
${ }^{3}$ Approximate $\Phi$ in $L^{2}(\Omega)$ by functions in $C_{c}^{1}(\Omega)$ and reason as for the estimate on (7.8) in the proof of Lemma 7.6.
} 
a.e. $t \in] 0, T\left[\left(^{4}\right)\right.$. By choice of $\nu_{K}$ and thanks to the estimate on $F$ in Proposition 3.1 and the fact that $2 d-2+\beta>0$, we have

$$
\begin{aligned}
& \sum_{n=1}^{N_{k}} k \sum_{K \in \mathcal{M}} \sum_{\sigma \in \mathcal{E}_{K}} \operatorname{diam}(K)^{d-1} \nu_{K} \mathrm{~m}(K)\left|F_{K, \sigma}^{n}\right|\left(\sum_{n=1}^{N_{k}} k \sum_{K \in \mathcal{M}} \sum_{\sigma \in \mathcal{E}_{K}} \mathrm{~m}(K)\right)^{\frac{1}{2}}\left(\sum_{n=1}^{N_{k}} k \sum_{K \in \mathcal{M}} \sum_{\sigma \in \mathcal{E}_{K}} \operatorname{diam}(K)^{2 d-2} \nu_{K}^{2} \mathrm{~m}(K)\left|F_{K, \sigma}^{n}\right|^{2}\right)^{\frac{1}{2}} \\
& \leq C_{28}\left(\sup _{n=1, \ldots, N_{k}} \sum_{K \in \mathcal{M}} \sum_{\sigma \in \mathcal{E}_{K}} \operatorname{diam}(K)^{2 d-2+\beta} \nu_{K} \mathrm{~m}(K)\left|F_{K, \sigma}^{n}\right|^{2}\right)^{\frac{1}{2}} \\
& \leq C_{29} \operatorname{size}(\mathcal{D})^{\frac{2 d-2+\beta}{2}} \rightarrow 0 \quad \operatorname{as} \operatorname{size}(\mathcal{D}) \rightarrow 0
\end{aligned}
$$

Hence, Lemma 7.4 shows that $\bar{p} \in L^{2}\left(0, T ; H^{1}(\Omega)\right)$ and that $\nabla \bar{p}=\overline{\mathbf{v}}$ (which implies that, in fact, $\bar{p} \in L^{\infty}\left(0, T ; H^{1}(\Omega)\right)$.

Let $A_{\mathcal{D}}: \Omega \times \mathbb{R} \rightarrow M_{d}(\mathbb{R})$ be the function defined by $A_{\mathcal{D}}(x, s)=A_{K}(s)$ whenever $s \in \mathbb{R}$ and $x$ belongs to $K \in \mathcal{M}$. We also define $\breve{c}:] 0, T\left[\times \Omega \rightarrow \mathbb{R}\right.$ by $\breve{c}=c_{K}^{n-1}$ on $\left[(n-1) k, n k\left[\times K\left(n=1, \ldots, N_{k}\right.\right.\right.$ and $\left.K \in \mathcal{M}\right) ;$ noticing that $\breve{c}=c_{K}^{0} \in[0,1]$ on $[0, k[\times K(K \in \mathcal{M})$ and that $\breve{c}=c(\cdot-k, \cdot)$ on $[k, T[\times \Omega$, it is clear that $\breve{c} \rightarrow \bar{c}$ in $L^{1}\left(0, T ; L_{\text {loc }}^{1}(\Omega)\right)$ as $k \rightarrow 0$ and $\operatorname{size}(\mathcal{D}) \rightarrow 0$. We have $\mathbf{U}=-A_{\mathcal{D}}(\cdot, \breve{c}) \mathbf{v}$ and thus, for all $\mathbf{Z} \in L^{2}(] 0, T[\times \Omega)^{d}, \int_{0}^{T} \int_{\Omega} \mathbf{Z} \cdot \mathbf{U}=\int_{0}^{T} \int_{\Omega}-A_{\mathcal{D}}(\cdot, \breve{c})^{T} \mathbf{Z} \cdot \mathbf{v}$. Applying Lemma 7.6 (with $-A^{T}$ instead of $A$, $u^{m}=\breve{c}$ and $\mathbf{Z}^{m}$ constant equal to $\left.\mathbf{Z}\right)$, and since $\mathbf{v}$ converges to $\nabla \bar{p}$ weakly in $L^{2}(] 0, T[\times \Omega)^{d}$, we see that $\int_{0}^{T} \int_{\Omega} \mathbf{Z} \cdot \mathbf{U} \rightarrow \int_{0}^{T} \int_{\Omega}-A(\cdot, \bar{c})^{T} \mathbf{Z} \cdot \nabla \bar{p}$, which proves that $\mathbf{U} \rightarrow \overline{\mathbf{U}}=-A(\cdot, \bar{c}) \nabla \bar{p}$ weakly in $L^{2}(] 0, T[\times \Omega)^{d}$ (since $\mathbf{U}$ is bounded in $L^{\infty}\left(0, T ; L^{2}(\Omega)\right)^{d}$, the convergence also holds weakly-* in this last space).

Let us now prove that $\bar{p}$ is the weak solution to (1.7) with $\bar{c}$ fixed above. As usual, the proof consists in multiplying the scheme by some test functions and passing to the limit. Let $\varphi \in C^{\infty}([0, T] \times \bar{\Omega})$ and define $\varphi^{n}(x)=\frac{1}{k} \int_{(n-1) k}^{n k} \varphi(t, x) d t$ for $n=1, \ldots, N_{k}$. Multiply $(2.6)$ by $\varphi^{n}\left(\mathbf{x}_{K}\right)$, sum on the control volumes and, using (2.3) and (2.8), gather by edges:

$$
\sum_{K \in \mathcal{M}} \mathrm{m}(K)\left(q_{K}^{+, n}-q_{K}^{-, n}\right) \varphi^{n}\left(\mathbf{x}_{K}\right)=\sum_{\sigma=K \mid L \in \mathcal{E}_{\text {int }}} F_{K, \sigma}^{n}\left(\varphi^{n}\left(\mathbf{x}_{L}\right)-\varphi^{n}\left(\mathbf{x}_{K}\right)\right) .
$$

Since $\varphi$ is regular, we have

$$
\begin{gathered}
\varphi^{n}\left(\mathbf{x}_{L}\right)-\varphi^{n}\left(\mathbf{x}_{K}\right)=\nabla \varphi^{n}\left(\mathbf{x}_{K}\right) \cdot\left(\mathbf{x}_{\sigma}-\mathbf{x}_{K}\right)+\nabla \varphi^{n}\left(\mathbf{x}_{L}\right) \cdot\left(\mathbf{x}_{L}-\mathbf{x}_{\sigma}\right)+R_{K, \sigma}^{n}-R_{L, \sigma}^{n} \\
\text { with }\left|R_{K, \sigma}^{n}\right| \leq C_{30} \operatorname{diam}(K)^{2},
\end{gathered}
$$

where $C_{30}$ does not depend on $n, \sigma=K \mid L, k$ or $\mathcal{D}$. Hence, gathering by control volumes (and still using the conservativity of the fluxes and the boundary conditions),

$$
\begin{aligned}
\sum_{K \in \mathcal{M}} \mathrm{m}(K)\left(q_{K}^{+, n}-q_{K}^{-, n}\right) \varphi^{n}\left(\mathbf{x}_{K}\right)= & \sum_{\sigma=K \mid L \in \mathcal{E}_{\mathrm{int}}} F_{K, \sigma}^{n}\left(\nabla \varphi^{n}\left(\mathbf{x}_{K}\right) \cdot\left(\mathbf{x}_{\sigma}-\mathbf{x}_{K}\right)+\nabla \varphi^{n}\left(\mathbf{x}_{L}\right) \cdot\left(\mathbf{x}_{L}-\mathbf{x}_{\sigma}\right)\right) \\
& +\sum_{\sigma=K \mid L \in \mathcal{E}_{\mathrm{int}}} F_{K, \sigma}^{n}\left(R_{K, \sigma}^{n}-R_{L, \sigma}^{n}\right) \\
= & \sum_{K \in \mathcal{M}} \nabla \varphi^{n}\left(\mathbf{x}_{K}\right) \cdot \sum_{\sigma \in \mathcal{E}_{K}} F_{K, \sigma}^{n}\left(\mathbf{x}_{\sigma}-\mathbf{x}_{K}\right)+\sum_{K \in \mathcal{M}} \sum_{\sigma \in \mathcal{E}_{K}} F_{K, \sigma}^{n} R_{K, \sigma}^{n} \\
= & -\sum_{K \in \mathcal{M}} \mathrm{m}(K) \nabla \varphi^{n}\left(\mathbf{x}_{K}\right) \cdot \mathbf{U}_{K}^{n}+\sum_{K \in \mathcal{M}} \sum_{\sigma \in \mathcal{E}_{K}} F_{K, \sigma}^{n} R_{K, \sigma}^{n} \cdot
\end{aligned}
$$

\footnotetext{
${ }^{4}$ Multiply $\int_{\Omega} p(t, \cdot)=0$ by any function $\gamma(t) \in C_{c}^{\infty}(] 0, T[)$, integrate on $\left.t \in\right] 0, T[$ and pass to the limit; the equality $\int_{0}^{T} \int_{\Omega} \gamma(t) \bar{p}(t, x) d t d x=0$ for all regular $\gamma$ implies the result.
} 
If $\varphi_{k, \mathcal{D}}$ and $\Psi_{k, \mathcal{D}}$ denote the functions on $\left[0, T\left[\times \Omega\right.\right.$ which are equal to $\varphi^{n}\left(\mathbf{x}_{K}\right)$ and to $\nabla \varphi^{n}\left(\mathbf{x}_{K}\right)$ on $\left[(n-1) k, n k\left[\times K\right.\right.$, it is clear that $\varphi_{k, \mathcal{D}} \rightarrow \varphi$ and $\Psi_{k, \mathcal{D}} \rightarrow \nabla \varphi$ uniformly on $] 0, T[\times \Omega$ as $k \rightarrow 0$ and $\operatorname{size}(\mathcal{D}) \rightarrow 0$; multiplying (5.19) by $k$ and summing on $n=1, \ldots, N_{k}$, we obtain

$$
\int_{0}^{T} \int_{\Omega}\left(q^{+}-q^{-}\right) \varphi_{k, \mathcal{D}}=-\int_{0}^{T} \int_{\Omega} \Psi_{k, \mathcal{D}} \cdot \mathbf{U}+\sum_{n=1}^{N_{k}} k \sum_{K \in \mathcal{M}} \sum_{\sigma \in \mathcal{E}_{K}} F_{K, \sigma}^{n} R_{K, \sigma}^{n} .
$$

We have, by Proposition 3.1 and thanks to (5.6),

$$
\begin{aligned}
\left|\sum_{K \in \mathcal{M}} \sum_{\sigma \in \mathcal{E}_{K}} F_{K, \sigma}^{n} R_{K, \sigma}^{n}\right| & \leq C_{30} \sum_{K \in \mathcal{M}} \sum_{\sigma \in \mathcal{E}_{K}} \operatorname{diam}(K)^{2}\left|F_{K, \sigma}^{n}\right| \\
& \leq C_{30}\left(\sum_{K \in \mathcal{M}} \sum_{\sigma \in \mathcal{E}_{K}} \frac{\operatorname{diam}(K)^{4}}{\nu_{K} \mathrm{~m}(K)}\right)^{\frac{1}{2}}\left(\sum_{K \in \mathcal{M}} \sum_{\sigma \in \mathcal{E}_{K}} \nu_{K} \mathrm{~m}(K)\left|F_{K, \sigma}^{n}\right|^{2}\right)^{\frac{1}{2}} \\
& \leq C_{31}\left(\sum_{K \in \mathcal{M}} \mathrm{m}(K) \operatorname{diam}(K)^{4-2 d-\beta}\right)^{\frac{1}{2}} \\
& \leq C_{32} \operatorname{size}(\mathcal{D})^{\frac{4-2 d-\beta}{2}} \rightarrow 0 \quad \text { as } \operatorname{size}(\mathcal{D}) \rightarrow 0 .
\end{aligned}
$$

Hence, by the weak convergence of $\mathbf{U}$, we can pass to the limit in $(5.20)$ and we find $\int_{0}^{T} \int_{\Omega}\left(q^{+}-q^{-}\right) \varphi=$ $-\int_{0}^{T} \int_{\Omega} \nabla \varphi \cdot \overline{\mathbf{U}} ;$ since this equation is satisfied for all $\varphi \in C^{\infty}([0, T] \times \bar{\Omega})$, this concludes the proof that $\bar{p}$ is the weak solution to (1.7), for the given $\bar{c}$ (limit of $c$ ).

We now want to prove the strong convergence of $\mathbf{v}$ to $\nabla \bar{p}$ in $L^{2}(] 0, T[\times \Omega)^{d}$. To do so, we use (2.5) and (2.4) in (3.1), which we then multiply by $k$ and sum on $n=1, \ldots, N_{k}$; this leads to

$$
\int_{0}^{T} \int_{\Omega}\left(q^{+}-q^{-}\right) p=\int_{0}^{T} \int_{\Omega} A(\cdot, \breve{c}) \mathbf{v} \cdot \mathbf{v}+\sum_{n=1}^{N_{k}} k \sum_{K \in \mathcal{M}} \sum_{\sigma \in \mathcal{E}_{K}} \nu_{K} \mathrm{~m}(K)\left|F_{K, \sigma}^{n}\right|^{2} .
$$

Dropping the last term (which is nonnegative), the weak convergence of $p$ gives, since $\bar{p}$ is a solution to (1.7),

$$
\limsup _{k \rightarrow 0, \operatorname{size}(\mathcal{D}) \rightarrow 0} \int_{0}^{T} \int_{\Omega} A(\cdot, \breve{c}) \mathbf{v} \cdot \mathbf{v} \leq \int_{0}^{T} \int_{\Omega}\left(q^{+}-q^{-}\right) \bar{p}=\int_{0}^{T} \int_{\Omega} A(\cdot, \bar{c}) \nabla \bar{p} \cdot \nabla \bar{p}
$$

(the last equality is obtained using $\bar{p}$ as a test function in (1.7), which is possible since the weak formulation of (1.7) is in fact valid with test functions in $L^{1}\left(0, T ; H^{1}(\Omega)\right)$ ). We now write, thanks to (1.10),

$$
\begin{aligned}
\alpha_{A} \int_{0}^{T} \int_{\Omega}|\mathbf{v}-\nabla \bar{p}|^{2} \leq & \int_{0}^{T} \int_{\Omega} A(\cdot, \breve{c})(\mathbf{v}-\nabla \bar{p}) \cdot(\mathbf{v}-\nabla \bar{p}) \\
= & \int_{0}^{T} \int_{\Omega} A(\cdot, \breve{c}) \mathbf{v} \cdot \mathbf{v}-\int_{0}^{T} \int_{\Omega} A(\cdot, \breve{c}) \mathbf{v} \cdot \nabla \bar{p}-\int_{0}^{T} \int_{\Omega} A(\cdot, \breve{c}) \nabla \bar{p} \cdot \mathbf{v} \\
& +\int_{0}^{T} \int_{\Omega} A(\cdot, \breve{c}) \nabla \bar{p} \cdot \nabla \bar{p} .
\end{aligned}
$$

Up to a subsequence, we can assume that $\breve{c} \rightarrow \bar{c}$ a.e. on $] 0, T[\times \Omega$ and (1.10) then gives $A(\cdot, \breve{c}) \nabla \bar{p} \rightarrow$ $A(\cdot, \bar{c}) \nabla \bar{p}$ and $A(\cdot, \breve{c})^{T} \nabla \bar{p} \rightarrow A(\cdot, \bar{c})^{T} \nabla \bar{p}$ strongly in $L^{2}(] 0, T[\times \Omega)^{d}$. Hence, the weak convergence of $\mathbf{v}$ to $\nabla \bar{p}$ allows to pass to the limit in the second and third terms of the right-hand side of (5.24); the last term of this right-hand side obviously converges and (5.23) therefore gives

$$
\limsup _{k \rightarrow 0, \operatorname{size}(\mathcal{D}) \rightarrow 0} \alpha_{A} \int_{0}^{T} \int_{\Omega}|\mathbf{v}-\nabla \bar{p}|^{2} \leq \limsup _{k \rightarrow 0, \operatorname{size}(\mathcal{D}) \rightarrow 0} \int_{0}^{T} \int_{\Omega} A(\cdot, \breve{c}) \mathbf{v} \cdot \mathbf{v}-\int_{0}^{T} \int_{\Omega} A(\cdot, \bar{c}) \nabla \bar{p} \cdot \nabla \bar{p} \leq 0,
$$


which concludes the proof of the strong convergence of $\mathbf{v}$ to $\nabla \bar{p}$ in $L^{2}(] 0, T[\times \Omega)^{d}$. The strong convergence of $\mathbf{U}$ in the same space is then a consequence of Lemma 7.6 , of the equality $\mathbf{U}=-A_{\mathcal{D}}(\cdot, \breve{c}) \mathbf{v}$ and of the strong convergence of $\mathbf{v}$.

We conclude by proving that, up to subsequence and as $k \rightarrow 0$ and $\operatorname{size}(\mathcal{D}) \rightarrow 0, p(t) \rightarrow \bar{p}(t)$ in $L_{\text {loc }}^{1}(\Omega)$ for a.e. $t \in] 0, T$. Since $p$ is bounded in $L^{\infty}\left(0, T ; L^{2}(\Omega)\right)$, and thus in $L^{\infty}\left(0, T ; L_{\text {loc }}^{1}(\Omega)\right)$, this a.e. convergence and Vitali's theorem imply the convergence in $L^{1}\left(0, T ; L_{\text {loc }}^{1}(\Omega)\right)$ and, using once again the bound on $p$ in $L^{\infty}\left(0, T ; L^{2}(\Omega)\right)$, we deduce the strong convergences stated in Theorem 2.2.

As $\mathbf{v}$ converges in $L^{2}\left(0, T ; L^{2}(\Omega)\right)^{d}$, we can assume that, up to a subsequence, $\mathbf{v}(t) \rightarrow \nabla \bar{p}(t)$ in $L^{2}(\Omega)^{d}$ for a.e. $t \in] 0, T$. Take a $t_{0}$ for which this convergence holds, and such that $\int_{\Omega} \bar{p}\left(t_{0}\right)=0$; we now prove, by way of contradiction, that $p\left(t_{0}\right) \rightarrow \bar{p}\left(t_{0}\right)$ in $L_{\text {loc }}^{1}(\Omega)$ (along the same subsequence as the one chosen for $\mathbf{v}$, which thus does not depend on $t_{0}$ ). If this convergence does not hold then we can assume, up to a new subsequence, that, for some $\eta>0$,

$$
d\left(p\left(t_{0}\right), \bar{p}\left(t_{0}\right)\right) \geq \eta
$$

where $d$ is the distance in $L_{\text {loc }}^{1}(\Omega)$. By $(2.2),\left(p\left(t_{0}\right), \mathbf{v}\left(t_{0}\right), F^{n\left(t_{0}, k\right)}\right) \in L_{\nu}(\mathcal{D})$ (where $n\left(t_{0}, k\right)$ is such that $\left.\left(n\left(t_{0}, k\right)-1\right) k \leq t_{0}<n\left(t_{0}, k\right) k\right)$ and Proposition 3.1 proves, with the help of Cauchy-Schwarz inequality as at the end of the proof of Lemma 5.1, that $M_{1}\left(\mathcal{D}, \nu, F^{n\left(t_{0}, k\right)}\right)$ (defined in Lemma 7.3) stays bounded; hence, since $p\left(t_{0}\right)$ is bounded in $L^{2}(\Omega)$ (see again Proposition 3.1), Lemma 7.3 and Kolmogorov's compactness theorem show that, up to a subsequence, $p\left(t_{0}\right)$ converges to some $P$ strongly in $L_{\text {loc }}^{1}(\Omega)$ and weakly in $L^{2}(\Omega)$. By $(2.7)$, it is clear that $\int_{\Omega} P=0$ (use the weak convergence in $L^{2}(\Omega)$ ). Applying Lemma 7.4 to the functions constant in time $(u, \mathbf{r})=\left(p\left(t_{0}\right), \mathbf{v}\left(t_{0}\right)\right)$ and to the fluxes $H=F^{n\left(t_{0}, k\right)}$, the estimates in Proposition 3.1 allow to see that (7.5) is satisfied and thus that $\nabla P=\nabla \bar{p}\left(t_{0}\right)$ (because $\left.\mathbf{v}\left(t_{0}\right) \rightarrow \nabla \bar{p}\left(t_{0}\right)\right)$; hence, since $\int_{\Omega} \bar{p}\left(t_{0}\right)=0$, we deduce that $P=\bar{p}\left(t_{0}\right)$, and therefore that $p\left(t_{0}\right) \rightarrow \bar{p}\left(t_{0}\right)$ in $L_{\text {loc }}^{1}(\Omega)$. Since the subsequence along which this convergence holds has been extracted from a sequence which satisfies (5.25), this gives the contradiction we sought.

Remark 5.2 From the strong convergence of $\mathbf{v}$ and the a.e. convergence of $\breve{c}$, we have $\int_{0}^{T} \int_{\Omega} A(\cdot, \breve{c}) \mathbf{v} \cdot \mathbf{v} \rightarrow$ $\int_{0}^{T} \int_{\Omega} A(\cdot, \bar{c}) \nabla \bar{p} \cdot \nabla \bar{p}=\int_{0}^{T} \int_{\Omega}\left(q^{+}-q^{-}\right) \bar{p}$. Hence, (5.22) implies

$$
\lim _{k \rightarrow 0, \operatorname{size}(\mathcal{D}) \rightarrow 0} \sum_{n=1}^{N_{k}} k \sum_{K \in \mathcal{M}} \sum_{\sigma \in \mathcal{E}_{K}} \nu_{K} \mathrm{~m}(K)\left|F_{K, \sigma}^{n}\right|^{2}=0 .
$$

This will be useful in the next section.

\subsection{Convergence of the concentration}

Let us now turn to the convergence of $(c, \mathbf{w})$. By the estimates of Proposition 3.2, the convergence of $c$ to $\bar{c}$ not only holds in $L^{1}\left(0, T ; L_{\text {loc }}^{1}(\Omega)\right)$, but also in $L^{\infty}\left(0, T ; L^{2}(\Omega)\right)$ weak-* and strongly in $L^{p}\left(0, T ; L^{q}(\Omega)\right)$ for all $p<\infty$ and $q<2$. Up to a subsequence, we can assume that $\mathbf{w} \rightarrow \overline{\mathbf{w}}$ weakly in $L^{2}(] 0, T[\times \Omega)^{d}$. Thanks to the estimates on $G$ from Proposition 3.2, the analog of (5.17) writes:

$$
\begin{aligned}
\sum_{k=1}^{N_{k}} k \sum_{K \in \mathcal{M}} \sum_{\sigma \in \mathcal{E}_{K}} \operatorname{diam}(K)^{d-1} \nu_{K} \mathrm{~m}(K)\left|G_{K, \sigma}^{n}\right| \\
\leq C_{33}\left(\sum_{k=1}^{N_{k}} k \sum_{K \in \mathcal{M}} \sum_{\sigma \in \mathcal{E}_{K}} \operatorname{diam}(K)^{2 d-2+\beta} \nu_{K} \mathrm{~m}(K)\left|G_{K, \sigma}^{n}\right|^{2}\right)^{\frac{1}{2}} \\
\leq C_{34} \operatorname{size}(\mathcal{D})^{\frac{2 d-2+\beta}{2}} \rightarrow 0 \quad \text { as } \operatorname{size}(\mathcal{D}) \rightarrow 0
\end{aligned}
$$

Hence, by (2.9) and Lemma 7.4, we have $\bar{c} \in L^{2}\left(0, T ; H^{1}(\Omega)\right)$ and $\overline{\mathbf{w}}=\nabla \bar{c}$. 
We now prove that $\bar{c}$ is a solution to (1.8), with $\overline{\mathbf{U}}$ the strong limit of $\mathbf{U}$ found in Section 5.2. Let $\varphi \in C_{c}^{\infty}\left(\left[0, T[\times \bar{\Omega})\right.\right.$ and, for $n=1, \ldots, N_{k}, \varphi^{n}(x)=\frac{1}{k} \int_{(n-1) k}^{n k} \varphi(t, x) d t$. We multiply $(2.12)$ by $k \varphi^{n}\left(\mathbf{x}_{K}\right)$ and sum on $K \in \mathcal{M}$ and on $n=1, \ldots, N_{k}$; this gives $T_{6}+T_{7}+T_{8}+T_{9}=T_{10}$. Let us study the limit of each of these terms, as $k \rightarrow 0$ and $\operatorname{size}(\mathcal{D}) \rightarrow 0$.

\subsubsection{Limit of $T_{6}$}

We have, since $\varphi^{N_{k}}=\varphi^{N_{k}+1}=0$ for $k$ small enough (the support of $\varphi$ does not touch $t=T$ ),

$$
\begin{aligned}
T_{6} & =\sum_{n=1}^{N_{k}} k \sum_{K \in \mathcal{M}} \mathrm{m}(K) \Phi_{K} \frac{c_{K}^{n}-c_{K}^{n-1}}{k} \varphi^{n}\left(\mathbf{x}_{K}\right) \\
& =\sum_{n=1}^{N_{k}} k \sum_{K \in \mathcal{M}} \mathrm{m}(K) \Phi_{K} c_{K}^{n} \frac{\varphi^{n}\left(\mathbf{x}_{K}\right)-\varphi^{n+1}\left(\mathbf{x}_{K}\right)}{k}-\sum_{K \in \mathcal{M}} \mathrm{m}(K) \Phi_{K} c_{K}^{0} \varphi^{1}\left(\mathbf{x}_{K}\right) \\
& =\int_{0}^{T} \int_{\Omega} \Phi c \zeta_{k, \mathcal{D}}-\int_{\Omega} \Phi_{\mathcal{D}} c_{0} \pi_{k, \mathcal{D}}
\end{aligned}
$$

where $\Phi_{\mathcal{D}}=\Phi_{K}$ on $K$ (as before), $\zeta_{k, \mathcal{D}}=\frac{\varphi^{n}\left(\mathbf{x}_{K}\right)-\varphi^{n+1}\left(\mathbf{x}_{K}\right)}{k}$ on $\left[(n-1) k, n k\left[\times K\right.\right.$ and $\pi_{k, \mathcal{D}}=\varphi_{K}^{1}$ on $K$ $\left(n=1, \ldots, N_{k}\right.$ and $\left.K \in \mathcal{M}\right)$. By regularity of $\varphi$, it is clear that $\zeta_{k, \mathcal{D}} \rightarrow-\partial_{t} \varphi$ uniformly on $[0, T] \times \Omega$ and $\pi_{k, \mathcal{D}} \rightarrow \varphi(0, \cdot)$ uniformly on $\Omega$; we also recall that $\Phi_{\mathcal{D}} \rightarrow \Phi$ strongly in $L^{2}(\Omega)$. The weak-* convergence of $c$ in $L^{\infty}\left(0, T ; L^{2}(\Omega)\right)$ then implies

$$
T_{6} \rightarrow-\int_{0}^{T} \int_{\Omega} \Phi \bar{c} \partial_{t} \varphi-\int_{\Omega} \Phi c_{0} \varphi(0, \cdot) .
$$

\subsubsection{Limit of $T_{7}$}

Making use of manipulations which should be, at this stage, familiar to the reader, we have, using (5.18) and letting $\Psi_{k, \mathcal{D}}$ be the function on $\left[0, T\left[\times \Omega\right.\right.$ equal to $\nabla \varphi^{n}\left(\mathbf{x}_{K}\right)$ on $[(n-1) k, n k[\times K$,

$$
\begin{aligned}
T_{7} & =-\sum_{n=1}^{N_{k}} k \sum_{K \in \mathcal{M}} \sum_{\sigma \in \mathcal{E}_{K}} G_{K, \sigma}^{n} \varphi^{n}\left(\mathbf{x}_{K}\right) \\
& =\sum_{n=1}^{N_{k}} k \sum_{\sigma=K \mid L \in \mathcal{E}_{\text {int }}} G_{K, \sigma}^{n}\left(\varphi^{n}\left(\mathbf{x}_{L}\right)-\varphi^{n}\left(\mathbf{x}_{K}\right)\right) \\
& =\sum_{n=1}^{N_{k}} k \sum_{K \in \mathcal{M}} \nabla \varphi^{n}\left(\mathbf{x}_{K}\right) \cdot \sum_{\sigma \in \mathcal{E}_{K}} G_{K, \sigma}^{n}\left(\mathbf{x}_{\sigma}-\mathbf{x}_{K}\right)+\sum_{n=1}^{N_{k}} k \sum_{K \in \mathcal{M}} \sum_{\sigma \in \mathcal{E}_{K}} G_{K, \sigma}^{n} R_{K, \sigma}^{n} \\
& =\sum_{n=1}^{N_{k}} k \sum_{K \in \mathcal{M}} \mathrm{m}(K) D_{K}\left(\mathbf{U}_{K}^{n}\right) \mathbf{w}_{K}^{n} \cdot \nabla \varphi^{n}\left(\mathbf{x}_{K}\right)+\sum_{n=1}^{N_{k}} k \sum_{K \in \mathcal{M}} \sum_{\sigma \in \mathcal{E}_{K}} G_{K, \sigma}^{n} R_{K, \sigma}^{n} \\
& =\int_{0}^{T} \int_{\Omega} D(\cdot, \mathbf{U}) \mathbf{w} \cdot \Psi_{k, \mathcal{D}}+\sum_{n=1}^{N_{k}} k \sum_{K \in \mathcal{M}} \sum_{\sigma \in \mathcal{E}_{K}} G_{K, \sigma}^{n} R_{K, \sigma}^{n} \\
& =\int_{0}^{T} \int_{\Omega} \mathbf{w} \cdot D(\cdot, \mathbf{U})^{T} \Psi_{k, \mathcal{D}}+\sum_{n=1}^{N_{k}} k \sum_{K \in \mathcal{M}} \sum_{\sigma \in \mathcal{E}_{K}} G_{K, \sigma}^{n} R_{K, \sigma}^{n} .
\end{aligned}
$$

But, thanks to the estimates on $G$ from Proposition 3.2, the analog of (5.21) writes

$$
\left|\sum_{n=1}^{N_{k}} k \sum_{K \in \mathcal{M}} \sum_{\sigma \in \mathcal{E}_{K}} G_{K, \sigma}^{n} R_{K, \sigma}^{n}\right| \leq C_{35} \operatorname{size}(\mathcal{D})^{\frac{4-2 d-\beta}{2}} \rightarrow 0 \quad \operatorname{as} \operatorname{size}(\mathcal{D}) \rightarrow 0 .
$$


Since $\mathbf{U} \rightarrow \overline{\mathbf{U}}$ strongly in $L^{2}(] 0, T[\times \Omega)^{d}$, hypothesis (1.11) classically implies that $D(\cdot, \mathbf{U}) \rightarrow D(\cdot, \overline{\mathbf{U}})$ strongly in $L^{2}(] 0, T[\times \Omega)^{d \times d}$ (extract a subsequence of $\mathbf{U}$ which converges a.e. and use Vitali's theorem). Since $\Psi_{k, \mathcal{D}} \rightarrow \nabla \varphi$ uniformly on $] 0, T\left[\times \Omega\right.$, we deduce that $D(\cdot, \mathbf{U})^{T} \Psi_{k, \mathcal{D}} \rightarrow D(\cdot, \overline{\mathbf{U}})^{T} \nabla \varphi$ in $L^{2}(] 0, T[\times \Omega)^{d}$ and the weak convergence of $\mathbf{w}$ to $\nabla \bar{c}$ allows to pass to the limit in (5.28):

$$
T_{7} \rightarrow \int_{0}^{T} \int_{\Omega} D(\cdot, \overline{\mathbf{U}}) \nabla \bar{c} \cdot \nabla \varphi
$$

\subsubsection{Limit of $T_{8}$}

The term $T_{8}$ is build writing $-k T_{2}$ (see page 13) with $\varphi^{n}\left(\mathbf{x}_{K}\right)$ instead of $\varphi_{K}$ and summing on $n$ :

$$
T_{8}=\sum_{n=1}^{N_{k}} k \sum_{K \in \mathcal{M}} \sum_{\sigma=K \mid L \in \mathcal{E}_{K} \cap \mathcal{E}_{\text {int }}}\left[\left(-F_{K, \sigma}^{n}\right)^{+} c_{K}^{n}-\left(-F_{L, \sigma}^{n}\right)^{-} c_{L}^{n}\right] \varphi^{n}\left(\mathbf{x}_{K}\right) .
$$

In the proof of Lemma 5.1, we obtained estimate (5.14) on $T_{2}$. This inequality has been proved for test functions $\varphi$ in $C_{c}^{2}(\Omega)$, but is also valid for test functions in $C^{2}(\bar{\Omega})$; in the same way, it is still valid if we use, in the definition of $T_{2}, \varphi^{n}\left(\mathbf{x}_{K}\right)$ rather than the mean value of $\varphi$ on $K$ (because (5.18) is similar to (5.2) without requiring $\mathbf{x}_{K}$ to be the barycenter of $K$ ). Therefore, thanks to the estimates of Proposition 3.2 ,

$$
\begin{aligned}
\mid T_{8}+ & \sum_{n=1}^{N_{k}} k \sum_{K \in \mathcal{M}} \mathrm{m}(K) c_{K}^{n} \mathbf{U}_{K}^{n} \cdot \nabla \varphi^{n}\left(\mathbf{x}_{K}\right) \mid \\
\leq & C_{36} \operatorname{size}(\mathcal{D})^{\frac{4-2 d-\beta}{2}} \sum_{n=1}^{N_{k}} k\left(\sum_{K \in \mathcal{M}} \mathrm{m}(K)\left(\left|\mathbf{w}_{K}^{n}\right|+\left|c_{K}^{n}\right|\right)^{2}\right)^{\frac{1}{2}} \\
& +C_{36} \sum_{n=1}^{N_{k}} k\left(\sum_{K \in \mathcal{M}} \sum_{\sigma \in \mathcal{E}_{K}} \nu_{K} \mathrm{~m}(K)\left|F_{K, \sigma}^{n}\right|^{2}\right)^{\frac{1}{2}}\left(\sum_{K \in \mathcal{M}} \mathrm{m}(K)\left|\mathbf{w}_{K}^{n}\right|^{2}\right)^{\frac{1}{2}} \\
& +C_{36} \operatorname{size}(\mathcal{D}) \sum_{n=1}^{N_{k}} k\left(\sum_{K \in \mathcal{M}} \sum_{\sigma \in \mathcal{E}_{K}} \nu_{K} \mathrm{~m}(K)\left|G_{K, \sigma}^{n}\right|^{2}\right)^{\frac{1}{2}} \\
\leq & C_{37}\left(\operatorname{size}(\mathcal{D})^{\frac{4-2 d-\beta}{2}}+\left(\sum_{n=1}^{N_{k}} k \sum_{K \in \mathcal{M}} \sum_{\sigma \in \mathcal{E}_{K}} \nu_{K} \mathrm{~m}(K)\left|F_{K, \sigma}^{n}\right|^{2}\right)^{\frac{1}{2}}+\operatorname{size}(\mathcal{D})\right)
\end{aligned}
$$

and, by (5.26), this last term tends to 0 as $k \rightarrow 0$ and $\operatorname{size}(\mathcal{D}) \rightarrow 0$. With the same $\Psi_{k, \mathcal{D}}$ as before, we have

$$
\sum_{n=1}^{N_{k}} k \sum_{K \in \mathcal{M}} \mathrm{m}(K) c_{K}^{n} \mathbf{U}_{K}^{n} \cdot \nabla \varphi^{n}\left(\mathbf{x}_{K}\right)=\int_{0}^{T} \int_{\Omega} c \mathbf{U} \cdot \Psi_{k, \mathcal{D}}
$$

and we therefore can pass to the limit (using the weak convergence of $c$ in $L^{2}(] 0, T[\times \Omega)$, the strong convergence of $\mathbf{U}$ in $L^{2}(] 0, T[\times \Omega)^{d}$ and the uniform convergence of $\Psi_{k, \mathcal{D}}$ on $] 0, T[\times \Omega)$ to obtain

$$
T_{8} \rightarrow-\int_{0}^{T} \int_{\Omega} \bar{c} \overline{\mathbf{U}} \cdot \nabla \varphi .
$$

\subsubsection{Limits of $T_{9}$ and $T_{10}$}

We have, with $\varphi_{k, \mathcal{D}}$ equal to $\varphi^{n}\left(\mathbf{x}_{K}\right)$ on $[(n-1) k, n k[\times K$,

$$
T_{9}=\sum_{n=1}^{N_{k}} k \sum_{K \in \mathcal{M}} \mathrm{m}(K) q_{K}^{-, n} c_{K}^{n} \varphi^{n}\left(\mathbf{x}_{K}\right)=\int_{0}^{T} \int_{\Omega} q^{-} c \varphi_{k, \mathcal{D}} \rightarrow \int_{0}^{T} \int_{\Omega} q^{-} \bar{c} \varphi .
$$


It is also easy to pass to the limit in

$$
T_{10}=\sum_{n=1}^{N_{k}} k \sum_{K \in \mathcal{M}} \mathrm{m}(K) q_{K}^{+, n} \widehat{c}_{K}^{n} \varphi^{n}\left(\mathbf{x}_{K}\right)=\int_{0}^{T} \int_{\Omega} q^{+} \widehat{c}_{k, \mathcal{D}} \varphi_{k, \mathcal{D}}
$$

once we notice that, as for $\Phi_{\mathcal{D}}$, the function $\widehat{c}_{k, \mathcal{D}}$ equal to $\widehat{c}_{K}^{n}$ on $[(n-1) k, n k[\times K$ converges to $\widehat{c}$ in $L^{2}(] 0, T[\times \Omega)$. Hence,

$$
T_{10} \rightarrow \int_{0}^{T} \int_{\Omega} q^{+} \widehat{c} \varphi
$$

Gathering (5.27), (5.29), (5.30), (5.31) and (5.32) in $T_{6}+T_{7}+T_{8}+T_{9}=T_{10}$, we deduce that $\bar{c}$ is a weak solution to (1.8) with the function $\overline{\mathbf{U}}$ limit of $\mathbf{U}$.

\section{$6 \quad$ Numerical results}

In this section, we illustrate the behavior of the mixed finite volume scheme by applying it to the system (1.1) - (1.6), which describes the miscible displacement of one fluid by another in a porous medium. Some of the tests cases come from the paper [20] where an ELLAM-MFEM scheme is used, and our results compare very well to the ones in this reference.

In all the test cases, the spatial domain is $\Omega=(0,1000) \times(0,1000) \mathrm{ft}^{2}$ and the time period is $[0,3600]$ days. The injection well is located at the upper-right corner $(1000,1000)$ with an injection rate $q^{+}=30 \mathrm{ft}^{2} /$ day and an injection concentration $\hat{c}=1.0$. The production well is located at the lower-left corner $(0,0)$ with a production rate $q^{-}=30 \mathrm{ft}^{2} /$ day. The viscosity of the oil is $\mu(0)=1.0 \mathrm{cp}$, the porosity of the medium is specified as $\Phi(x)=0.1$ and the initial concentration is $c_{0}(x)=0$.

Remark 6.1 Although this does not entirely satisfy the assumptions of our theoretical study, the wells can be considered as dirac masses; from the point of view of numerical tests, we saw no difference between using dirac masses for $q^{+}$and $q^{-}$or approximations of such masses by functions concentrated in small domains (which would be admissible in the theoretical study).

The mesh of the domain is made of 928 triangles of maximal edge length $50 \mathrm{ft}$. We take as time step $k=36$ days but the scheme still works with greater time steps (indeed, the discretization is implicit in time and does not require any stability condition). In fact, if we use the same time step $k=360$ days as in [20], we obtain numerical results close to the ones in this reference but, since the computational times are in any case very short (less than 3 seconds per time step on a personal computer), we choose the smaller time step $k=36$ days to show more accurate results with respect to the exact solution. As noticed in [9], the choice of $\nu_{K}$ has very little impact on the numerical outcomes and any small value for the penalization give good results; we therefore take $\nu_{K} \mathrm{~m}(K)=10^{-6}$ for all $K$.

For each test case, we present the surface plot and the contour plot of the concentration $c$, the interesting physical quantity, at $t=3$ years $(\approx 30$ time steps $)$ and $t=10$ years $(\approx 100$ time steps $)$.

Remark 6.2 Notice that our scheme preserves the discrete mass, that is to say, for $n=1, \ldots, N_{k}$,

$$
\int_{\Omega} \phi(x) c^{n}(x) d x+\int_{(n-1) k}^{n k} \int_{\Omega} q^{-}(t, x) c^{n}(x) d x d t=\int_{\Omega} \phi(x) c^{n-1}(x) d x+\int_{(n-1) k}^{n k} \int_{\Omega} q^{+}(t, x) \widehat{c}^{n}(x) d x d t
$$

(this is obtained by summing (2.12) on $K \in \mathcal{M}$ and using (2.10) and (2.13) to cancel the terms involving $G_{K, \sigma}^{n}$ and (2.3) to cancel the terms involving $F_{K, \sigma}^{n}$ ). We also want to point out that, in all the following numerical tests, our method does not induce neither overshoot nor undershoot on the concentration: the computed values for $c$ remain in $[0,1]$. 
Test 1. For this test case, we assume that the porous medium is homogeneous and isotropic: the permeability tensor is diagonal and constant, $\mathbf{K}=80 \mathbf{I}$. The mobility ratio between the resident and the injected fluids is $M=1$, so that the viscosity is constant $\mu(c)=1.0 \mathrm{cp}$.

We assume that $\Phi d_{m}=1.0 \mathrm{ft}^{2} /$ day, $\Phi d_{l}=5.0 \mathrm{ft}$ and $\Phi d_{t}=0.5 \mathrm{ft}$. This means that the diffusion effects will be considerably greater than the dispersion effects, which is in pratical unrealistic.

The surface plot and the contour plot of the concentration $c$ at $t=3$ years and $t=10$ years are shown in Figure 1. As expected, the Darcy velocity is radial and the contour plots are circular until the invading fluid reaches the production well (see at $t=3$ years). When the production well is reached, the invading fluid continues to fill the whole domain until $c=1$.

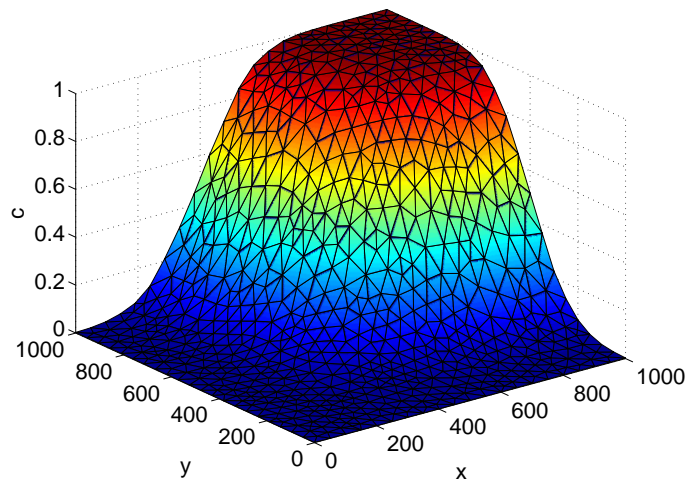

Surface plot at $t=3$ years

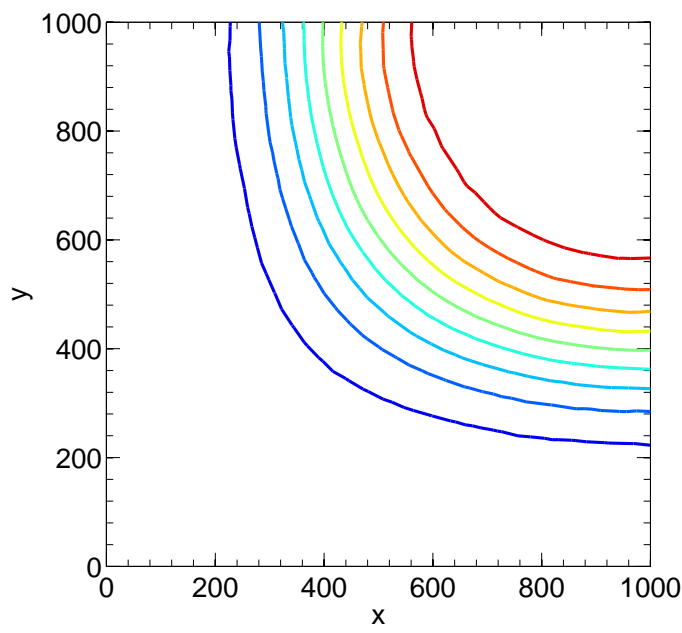

Contour plot at $t=3$ years

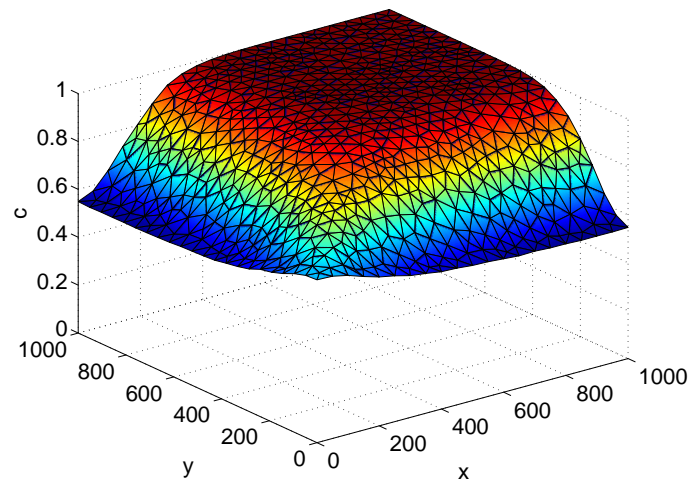

Surface plot at $t=10$ years

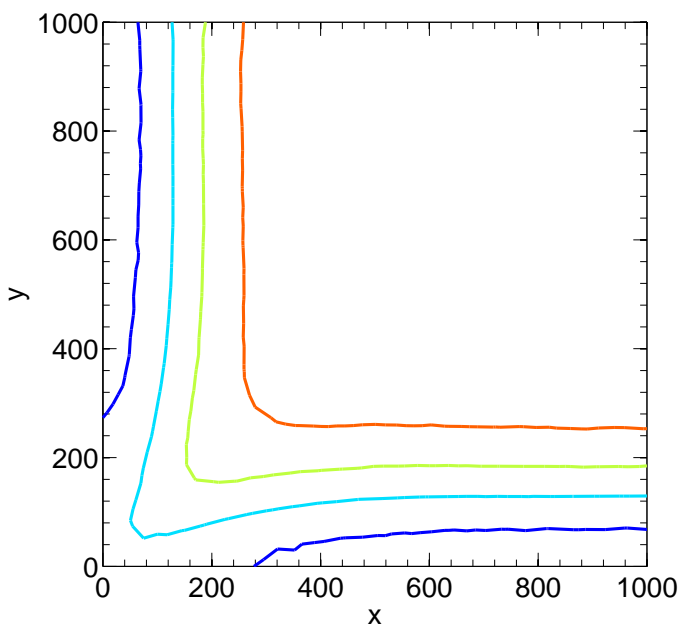

Contour plot at $t=10$ years

Figure 1: Concentration of the invading component in Test 1

Test 2. The permeability tensor is still diagonal and constant, $\mathbf{K}=80 \mathbf{I}$. The adverse mobility ratio is $M=41$ and the viscosity $\mu(c)$ now really depends on $c$.

We assume that there is no molecular diffusion $\Phi d_{m}=0.0 \mathrm{ft}^{2} /$ day and that $\Phi d_{l}=5.0 \mathrm{ft}$ and $\Phi d_{t}=0.5 \mathrm{ft}$. This means that we take into account dispersion effects, which is realistic. 
This test case is presented in [20] and permits to see the macroscopic fingering phenomenon. Indeed, the viscosity $\mu(c)$ rapidly changes across the fluid interface. It induces rapid changes of the Darcy velocity $\mathbf{U}$ and the difference between the longitudinal and the transverse dispersivity coefficients implies that the fluid flow is much faster along the diagonal direction. Such effects can be seen on the surface and contour plots in Figure 2.

Remark 6.3 Although this test (as well as tests 3 and 4) does not satisfy our theoretical assumptions (because $\left.d_{m}=0\right)$, we present its results to show that the mixed finite volume scheme is robust and can numerically handle more general cases than the ones admitted in the theoretical study, and also to compare it with other existing schemes for the same equations (note that there is no theoretical study of convergence whatsoever in [20] or [21]).

The study of the continuous problem (1.1) - (1.6) when $d_{m}=0$ demands quite evolved functional analysis (see [1]), and the translation of these techniques to the discrete case (to prove the convergence of the scheme in the case of no molecular diffusion) does not seem straightforward at all.

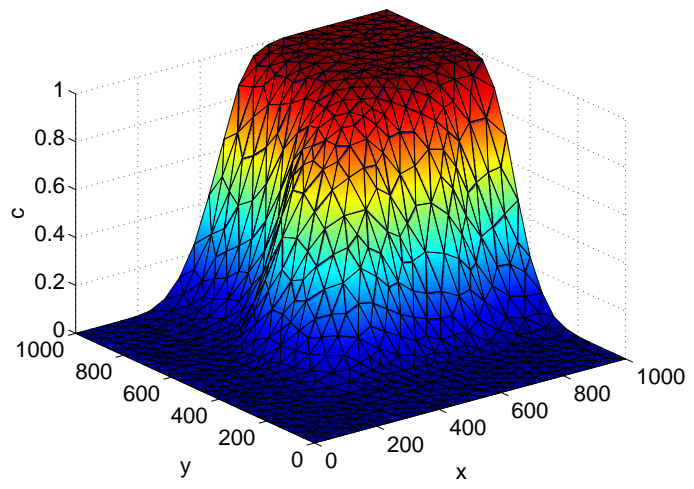

Surface plot at $t=3$ years

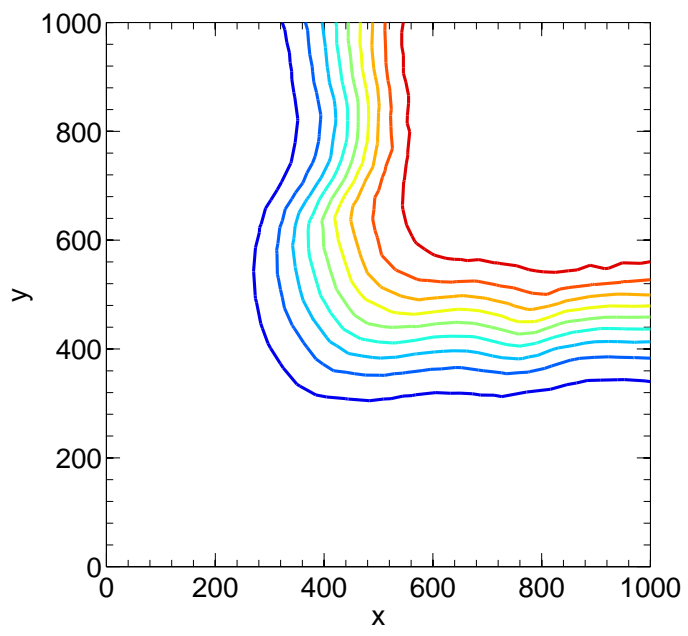

Contour plot at $t=3$ years

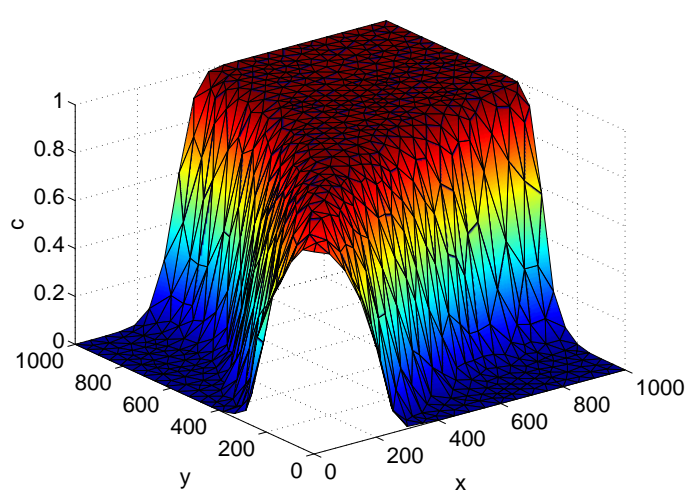

Surface plot at $t=10$ years

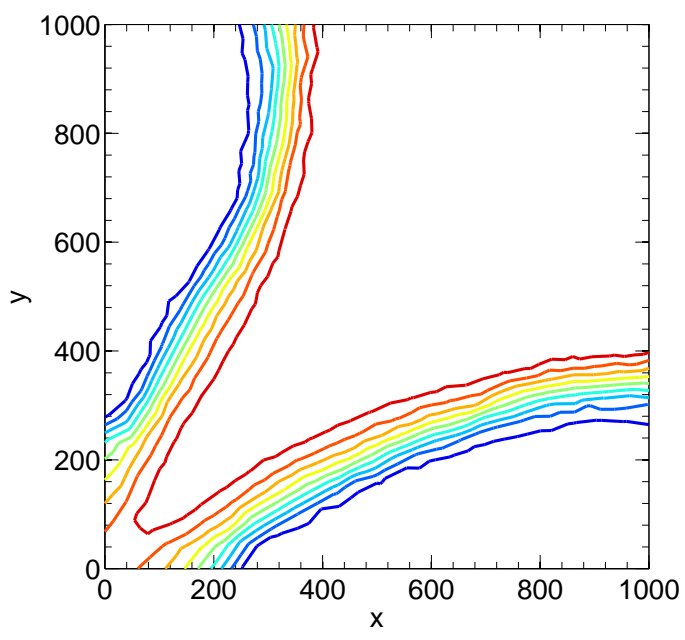

Contour plot at $t=10$ years

Figure 2: Concentration of the invading component in Test 2 
Test 3. In this test case, we consider that the permeability tensor is still diagonal but discontinuous: $\mathbf{K}=80 \mathbf{I}$ on the subdomain $(0,1000) \times(0,500)$ and $\mathbf{K}=20 \mathbf{I}$ on the subdomain $(0,1000) \times(500,1000)$. The adverse mobility ratio, the molecular diffusion, the longitudinal and the transverse dispersivities are the same as in Test 2.

The lower half domain has a larger permeability than the upper half domain. Therefore, when the invading fluid reaches the lower half domain, it "prefers" to pass through this domain rather than through the domain with lower permeability. As expected, we also notice that the upper half domain is, on the overall, less invaded than in Test 2. These effects are illustrated by the surface and contour plots of $c$ in Figure 3 .

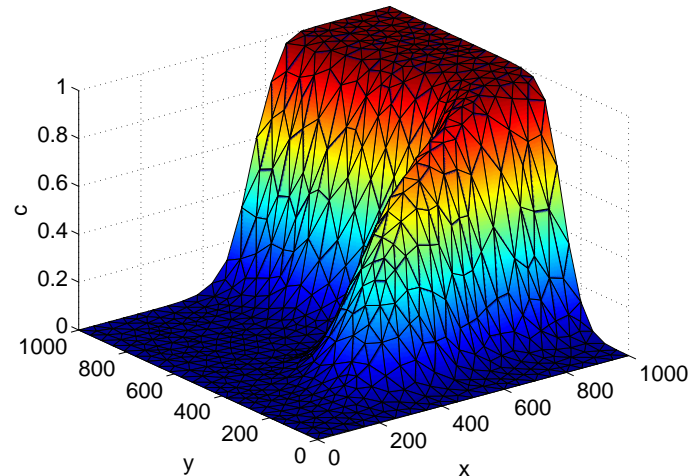

Surface plot at $t=3$ years

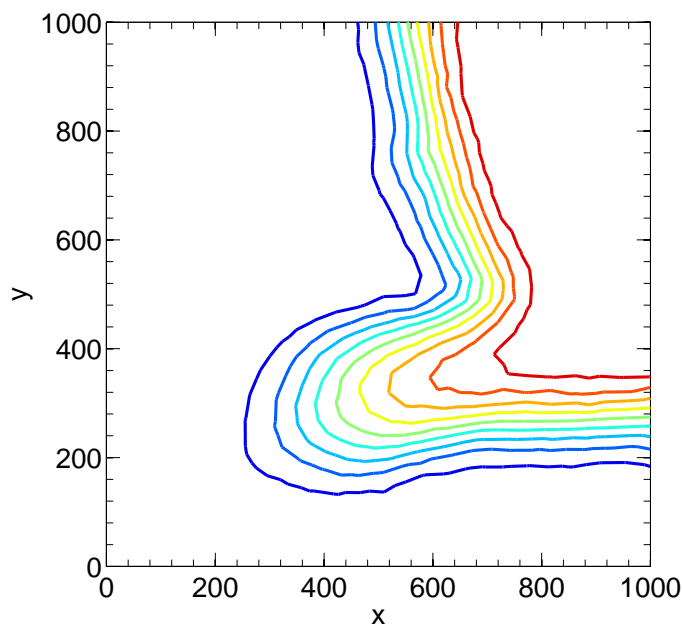

Contour plot at $t=3$ years

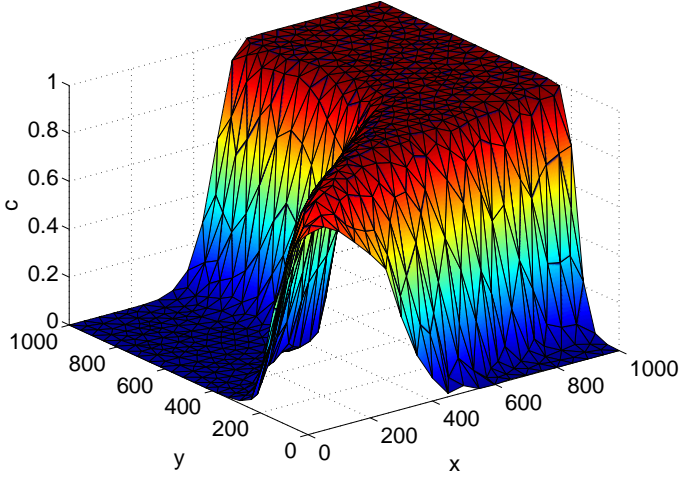

Surface plot at $t=10$ years

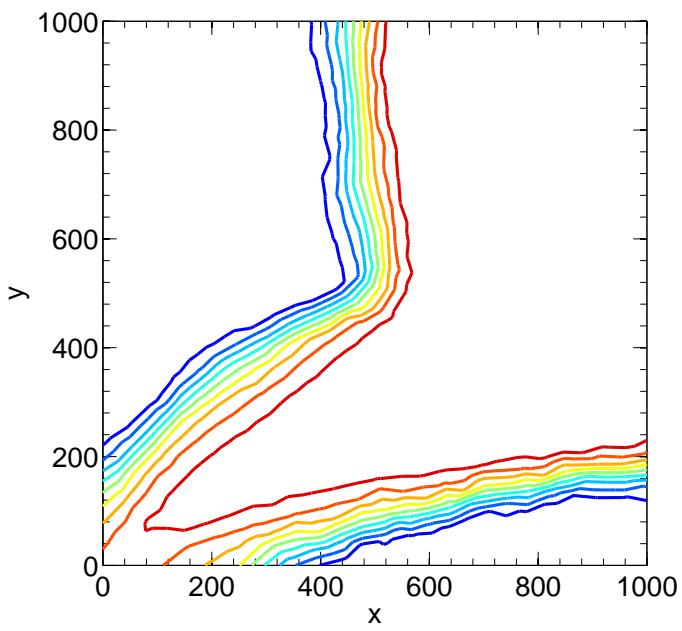

Contour plot at $t=10$ years

Figure 3: Concentration of the invading component in Test 3

Test 4. In this last test case, the permeability tensor has the form $\mathbf{K}=\kappa(x) \mathbf{I}$ with $\kappa(x)=80$ except on the four square subdomains $(200,400) \times(200,400),(600,800) \times(200,400),(200,400) \times(600,800)$, $(600,800) \times(600,800)$ where $\kappa(x)=20$. The adverse mobility ratio is $M=41$, and we take $\Phi d_{m}=$ $0.0 \mathrm{ft}^{2} /$ day, $\Phi d_{l}=5.0 \mathrm{ft}$ and $\Phi d_{t}=0.5 \mathrm{ft}$. 
Figure 4 shows the surface plot and the contour plot of the concentration at $t=3$ years and $t=10$ years. The subdomains where the permeability is lower can easily be seen on the figures. We note that the area occupied by the invading fluid at $t=10$ years is in this case larger than in Test 2 , where the permeability was homogeneous.

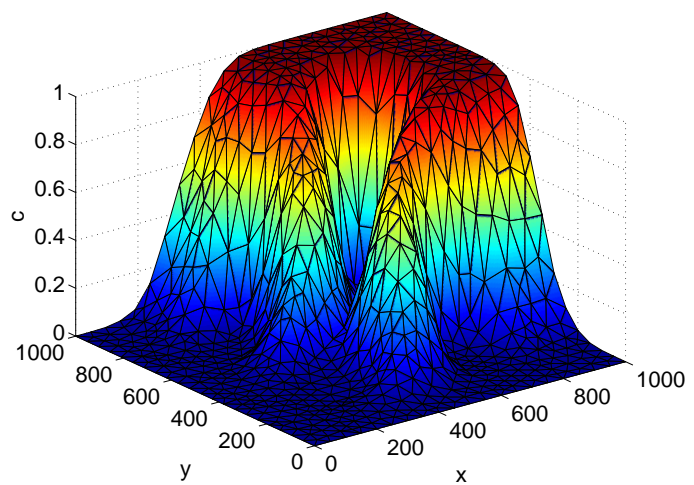

Surface plot at $t=3$ years

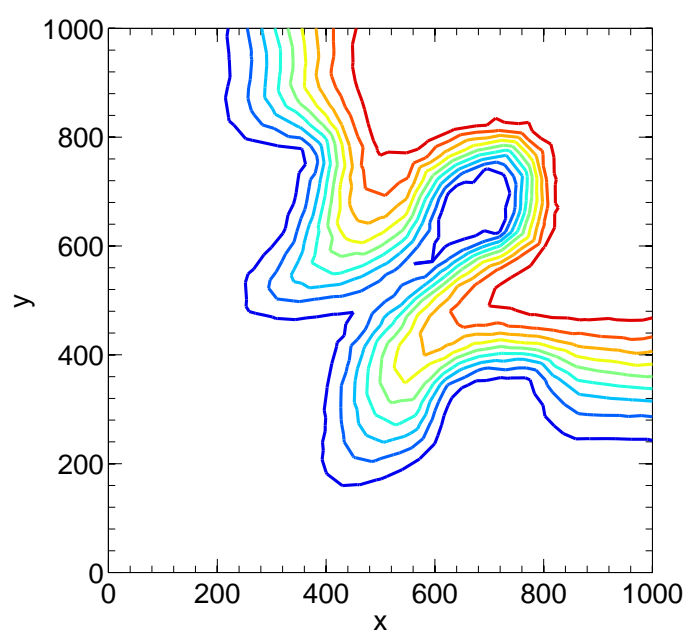

Contour plot at $t=3$ years

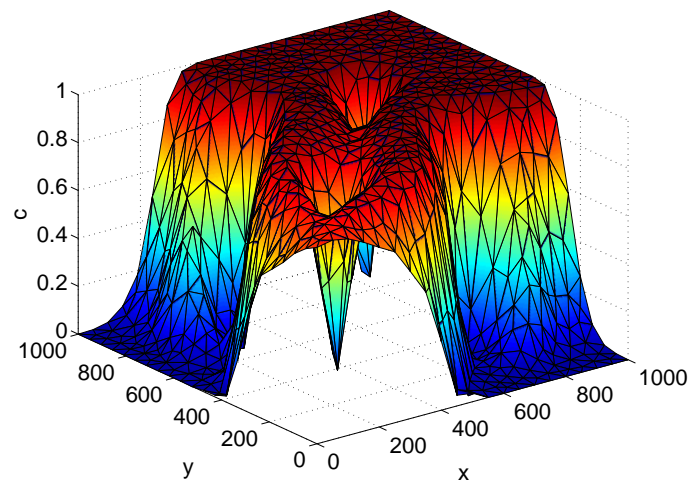

Surface plot at $t=10$ years

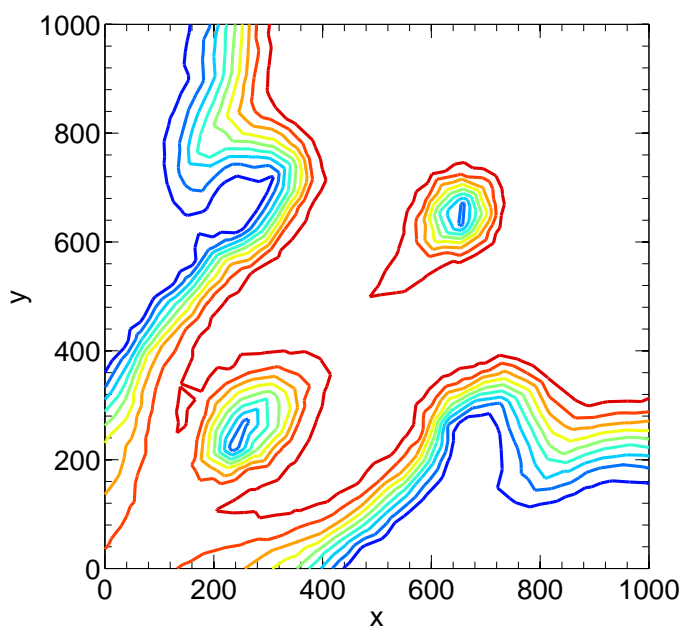

Contour plot at $t=10$ years

Figure 4: Concentration of the invading component in Test 4

\section{Appendix}

\subsection{A magical lemma}

The formula in the following lemma justifies equations such as (2.5) and (2.11). Its proof (a very simple application of Stoke's formula) can be found in [9].

Lemma 7.1 Let $K$ be a non empty polygonal convex open set in $\mathbb{R}^{d}$. For $\sigma \in \mathcal{E}_{K}$ (the edges of $K$ ), we define $\mathbf{x}_{\sigma}$ as the center of gravity of $\sigma$ and $\mathbf{n}_{K, \sigma}$ as the unit normal to $\sigma$ outward to $K$. Then, for all 
vector $\mathbf{e} \in \mathbb{R}^{d}$ and for all point $\mathbf{x}_{K} \in \mathbb{R}^{d}$, we have

$$
\mathrm{m}(K) \mathbf{e}=\sum_{\sigma \in \mathcal{E}_{K}} \mathrm{~m}(\sigma) \mathbf{e} \cdot \mathbf{n}_{K, \sigma}\left(\mathbf{x}_{\sigma}-\mathbf{x}_{K}\right)
$$

where $\mathrm{m}(K)$ is the d-dimensional measure of $K$ and $\mathrm{m}(\sigma)$ is the $(d-1)$-dimensional measure of $\sigma$.

\subsection{Lemmas on discrete gradients}

For $\mathcal{D}$ an admissible discretization of $\Omega$ and $\nu=\left(\nu_{K}\right)_{K \in \mathcal{M}}$ a family of positive numbers, we denote $L_{\nu}(\mathcal{D})$ the space of $(u, \mathbf{r}, H)$, with $u=\left(u_{K}\right)_{K \in \mathcal{M}}$ a family of numbers, $\mathbf{r}=\left(\mathbf{r}_{K}\right)_{K \in \mathcal{M}}$ a family of vectors and and $H=\left(H_{K, \sigma}\right)_{K \in \mathcal{M}, \sigma \in \mathcal{E}_{K}}$ a family of numbers, such that

$$
\mathbf{r}_{K} \cdot\left(\mathbf{x}_{\sigma}-\mathbf{x}_{K}\right)+\mathbf{r}_{L} \cdot\left(\mathbf{x}_{L}-\mathbf{x}_{\sigma}\right)+\nu_{K} \mathrm{~m}(K) H_{K, \sigma}-\nu_{L} \mathrm{~m}(L) H_{L, \sigma}=u_{L}-u_{K}, \forall \sigma=K \mid L \in \mathcal{E}_{\text {int }}
$$

(note that $u$ and $\mathbf{r}$ are also identified with the corresponding functions on $\Omega$ constant on each control volume $K$ ).

The following lemmas are the counterparts for Neumann boundary conditions of lemmas stated in [9] or [8] in the case of Dirichlet boundary conditions.

Lemma 7.2 Let $\Omega$ a convex polygonal bounded open subset of $\mathbb{R}^{d}, \mathcal{D}$ an admissible discretization of $\Omega$ such that $\operatorname{regul}(\mathcal{D}) \leq \theta$ for some $\theta>0$, and $\nu=\left(\nu_{K}\right)_{K \in \mathcal{M}}$ a family of positive numbers. Then there exists $C_{38}$ only depending on $d, \Omega$ and $\theta$ such that, for all $(u, \mathbf{r}, H) \in L_{\nu}(\mathcal{D})$ satisfying $\int_{\Omega} u=0$,

$$
\|u\|_{L^{2}(\Omega)} \leq C_{38}\left(\|\mathbf{r}\|_{L^{2}(\Omega)^{d}}+M_{2}(\mathcal{D}, \nu, H)\right)
$$

with $M_{2}(\mathcal{D}, \nu, H)=\left(\sum_{K \in \mathcal{M}} \sum_{\sigma \in \mathcal{E}_{K}} \operatorname{diam}(K)^{2 d-2} \nu_{K}^{2} \mathrm{~m}(K)\left|H_{K, \sigma}\right|^{2}\right)^{\frac{1}{2}}$.

\section{Proof of Lemma 7.2}

Let $w$ be the weak solution of $-\Delta w=u$ on $\Omega$ with homogeneous Neumann boundary conditions on $\partial \Omega$ (such a $w$ exists thanks to the fact that $\int_{\Omega} u=0$ ) and null mean value. Since $\Omega$ is convex, it is well known (see [16]) that $w \in H^{2}(\Omega)$ and that there exists $C_{39}$ only depending on $d$ and $\Omega$ such that

$$
\|w\|_{H^{2}(\Omega)} \leq C_{39}\|u\|_{L^{2}(\Omega)} .
$$

We multiply each equation of (7.1) by $\int_{\sigma} \nabla w \cdot \mathbf{n}_{K, \sigma} d \gamma$ and sum over the interior edges and gather by control volumes, using $\mathbf{n}_{K, \sigma}=-\mathbf{n}_{L, \sigma}$ whenever $\sigma=K \mid L$ :

$$
\begin{aligned}
& \sum_{K \in \mathcal{M}} \sum_{\sigma \in \mathcal{E}_{K} \cap \mathcal{E}_{\mathrm{int}}} \mathbf{r}_{K} \cdot\left(\mathbf{x}_{\sigma}-\mathbf{x}_{K}\right) \int_{\sigma} \nabla w \cdot \mathbf{n}_{K, \sigma} d \gamma \\
+ & \sum_{K \in \mathcal{M}} \sum_{\sigma \in \mathcal{E}_{K} \cap \mathcal{E}_{\mathrm{int}}} \nu_{K} \mathrm{~m}(K) H_{K, \sigma} \int_{\sigma} \nabla w \cdot \mathbf{n}_{K, \sigma} d \gamma=-\sum_{K \in \mathcal{M}} u_{K} \sum_{\sigma \in \mathcal{E}_{K} \cap \mathcal{E}_{\mathrm{int}}} \int_{\sigma} \nabla w \cdot \mathbf{n}_{K, \sigma} d \gamma .
\end{aligned}
$$

Since $\int_{\sigma} \nabla w \cdot \mathbf{n}_{K, \sigma} d \gamma=0$ whenever $\sigma \in \mathcal{E}_{\text {ext }}$, we can add to the preceding sums all the terms corresponding to boundary edges, which gives, by definition of $w$,

$$
\begin{aligned}
\sum_{K \in \mathcal{M}} \sum_{\sigma \in \mathcal{E}_{K}} \mathbf{r}_{K} \cdot\left(\mathbf{x}_{\sigma}-\mathbf{x}_{K}\right) \int_{\sigma} \nabla w \cdot \mathbf{n}_{K, \sigma} d \gamma & \\
+\sum_{K \in \mathcal{M}} \sum_{\sigma \in \mathcal{E}_{K}} \nu_{K} \mathrm{~m}(K) H_{K, \sigma} \int_{\sigma} \nabla w \cdot \mathbf{n}_{K, \sigma} d \gamma & =-\sum_{K \in \mathcal{M}} u_{K} \int_{\partial K} \nabla w \cdot \mathbf{n}_{K} d \gamma \\
& =-\sum_{K \in \mathcal{M}} u_{K} \int_{K} \Delta w \\
& =\sum_{K \in \mathcal{M}} \mathrm{m}(K) u_{K}^{2}=\|u\|_{L^{2}(\Omega)}^{2}
\end{aligned}
$$


Since $\operatorname{regul}(\mathcal{D}) \leq \theta,\left[8\right.$, Lemma 8.1] gives $C_{40}$ only depending on $d, \Omega$ and $\theta$ such that

$$
\left|\int_{\sigma} \nabla w d \gamma \cdot \mathbf{n}_{K, \sigma}\right|^{2} \leq\left|\int_{\sigma} \nabla w d \gamma\right|^{2} \leq \frac{C_{40} \mathrm{~m}(\sigma)}{\operatorname{diam}(K)}\|w\|_{H^{2}(K)}^{2} .
$$

Using Cauchy-Schwarz inequality, we deduce, $\operatorname{since} \operatorname{Card}\left(\mathcal{E}_{K}\right) \leq \operatorname{regul}(\mathcal{D}) \leq \theta$ for all $K \in \mathcal{M}$,

$$
\begin{aligned}
\sum_{K \in \mathcal{M}} \sum_{\sigma \in \mathcal{E}_{K}} \mathbf{r}_{K} \cdot\left(\mathbf{x}_{\sigma}-\mathbf{x}_{K}\right) \int_{\sigma} \nabla w \cdot \mathbf{n}_{K, \sigma} d \gamma \\
\leq\left(\sum_{K \in \mathcal{M}} \sum_{\sigma \in \mathcal{E}_{K}} \mathrm{~m}(K)\left|\mathbf{r}_{K}\right|^{2}\right)^{\frac{1}{2}}\left(\sum_{K \in \mathcal{M}} \sum_{\sigma \in \mathcal{E}_{K}} \frac{\operatorname{diam}(K)^{2}}{\mathrm{~m}(K)}\left|\int_{\sigma} \nabla w d \gamma \cdot \mathbf{n}_{K, \sigma}\right|^{2}\right)^{\frac{1}{2}} \\
\leq\left(C_{40} \theta\right)^{\frac{1}{2}}\left(\sum_{K \in \mathcal{M}} \mathrm{m}(K)\left|\mathbf{r}_{K}\right|^{2}\right)^{\frac{1}{2}}\left(\sum_{K \in \mathcal{M}} \sum_{\sigma \in \mathcal{E}_{K}} \frac{\operatorname{diam}(K) \mathrm{m}(\sigma)}{\mathrm{m}(K)}\|w\|_{H^{2}(K)}^{2}\right)^{\frac{1}{2}}
\end{aligned}
$$

We have, if $\sigma \in \mathcal{E}_{K}, \mathrm{~m}(\sigma) \leq \omega_{d-1} \operatorname{diam}(K)^{d-1}$ (where $\omega_{d-1}$ is the volume of the unit ball in $\mathbb{R}^{d-1}$ ); thus, by $(2.1), \frac{\operatorname{diam}(K) \mathrm{m}(\sigma)}{\mathrm{m}(K)} \leq \frac{\operatorname{regul}(\mathcal{D}) \omega_{d-1}}{\omega_{d}}$ and we obtain

$$
\sum_{K \in \mathcal{M}} \sum_{\sigma \in \mathcal{E}_{K}} \mathbf{r}_{K} \cdot\left(\mathbf{x}_{\sigma}-\mathbf{x}_{K}\right) \int_{\sigma} \nabla w \cdot \mathbf{n}_{K, \sigma} d \gamma \leq \frac{\theta^{\frac{3}{2}} \sqrt{C_{40} \omega_{d-1}}}{\sqrt{\omega_{d}}}\|\mathbf{r}\|_{L^{2}(\Omega)^{d}}\|w\|_{H^{2}(\Omega)} .
$$

Cauchy-Schwarz also gives

$$
\begin{aligned}
\sum_{K \in \mathcal{M}} \sum_{\sigma \in \mathcal{E}_{K}} \nu_{K} \mathrm{~m}(K) H_{K, \sigma} \int_{\sigma} \nabla w \cdot \mathbf{n}_{K, \sigma} d \gamma \\
\leq \quad\left(\sum_{K \in \mathcal{M}} \sum_{\sigma \in \mathcal{E}_{K}} \operatorname{diam}(K)^{2 d-2} \nu_{K}^{2} \mathrm{~m}(K)\left|H_{K, \sigma}\right|^{2}\right)^{\frac{1}{2}} \\
\times\left(\sum_{K \in \mathcal{M}} \sum_{\sigma \in \mathcal{E}_{K}} \frac{\mathrm{m}(K)}{\operatorname{diam}(K)^{2 d-2}}\left|\int_{\sigma} \nabla w \cdot \mathbf{n}_{K, \sigma} d \gamma\right|^{2}\right)^{\frac{1}{2}} \\
\leq M_{2}(\mathcal{D}, \nu, H)\left(\sum_{K \in \mathcal{M}} \sum_{\sigma \in \mathcal{E}_{K}} \frac{\mathrm{m}(\sigma) \mathrm{m}(K)}{\operatorname{diam}(K)^{2 d-1}}\|w\|_{H^{2}(K)}^{2}\right)^{\frac{1}{2}} .
\end{aligned}
$$

Since $\frac{\mathrm{m}(\sigma) \mathrm{m}(K)}{\operatorname{diam}(K)^{2 d-1}} \leq \omega_{d-1} \omega_{d}$, this inequality and (7.4) injected in (7.3) lead to

$$
\|u\|_{L^{2}(\Omega)}^{2} \leq C_{41}\|\mathbf{r}\|_{L^{2}(\Omega)^{d}}\|w\|_{H^{2}(\Omega)}+C_{41} M_{2}(\mathcal{D}, \nu, H)\|w\|_{H^{2}(\Omega)}
$$

with $C_{41}$ only depending on $d, \Omega$ and $\theta$, and we conclude thanks to (7.2).

Lemma 7.3 Let $\Omega$ be a convex polygonal bounded open subset of $\mathbb{R}^{d}, \mathcal{D}$ be an admissible discretization of $\Omega$ such that $\operatorname{regul}(\mathcal{D}) \leq \theta$ for some $\theta>0$ and $\nu=\left(\nu_{K}\right)_{K \in \mathcal{M}}$ be a family of positive numbers. Let $\omega$ be relatively compact in $\Omega$. Then there exists $C_{42}$ only depending on $d, \Omega, \omega$ and $\theta$ such that, for all $(u, \mathbf{r}, H) \in L_{\nu}(\mathcal{D})$ and all $|\xi|<\operatorname{dist}\left(\omega, \mathbb{R}^{d} \backslash \Omega\right)$,

$$
\|u(\cdot+\xi)-u\|_{L^{1}(\omega)} \leq C_{42}\left(\|\mathbf{r}\|_{L^{1}(\Omega)^{d}}+M_{1}(\mathcal{D}, \nu, H)\right)|\xi|
$$

where $M_{1}(\mathcal{D}, \nu, H)=\sum_{K \in \mathcal{M}} \sum_{\sigma \in \mathcal{E}_{K}} \operatorname{diam}(K)^{d-1} \nu_{K} \mathrm{~m}(K)\left|H_{K, \sigma}\right|$. 


\section{Proof of Lemma 7.3}

For $x \in \mathbb{R}^{d}, \xi \in \mathbb{R}^{d}$ and $\sigma \in \mathcal{E}$, we define $\chi(x, \sigma, \xi)=1$ if $\sigma \cap[x, x+\xi] \neq \emptyset$ and 0 otherwise. Let $x \in \omega$ and $\xi$ as in the lemma; we then have, since $[x, x+\xi] \subset \Omega$ (by choice of $\xi$ ), $|u(x+\xi)-u(x)| \leq$ $\sum_{\sigma=K \mid L \in \mathcal{E}_{\text {int }}} \chi(x, \sigma, \xi)\left|u_{K}-u_{L}\right|$ (in fact, this inequality only holds for a.e. $x \in \omega$, the points such that $x$ and $x+\xi$ do not belong to the boundary of a control volume and $[x, x+\xi]$ does not intersect the relative boundary of an edge). Hence, by (7.1),

$$
\begin{aligned}
|u(x+\xi)-u(x)| \leq & \sum_{\sigma=K \mid L \in \mathcal{E}_{\text {int }}}\left|\mathbf{r}_{K}\right| \operatorname{diam}(K) \chi(x, \sigma, \xi)+\left|\mathbf{r}_{L}\right| \operatorname{diam}(L) \chi(x, \sigma, \xi) \\
& +\sum_{\sigma=K \mid L \in \mathcal{E}_{\text {int }}} \nu_{K} \mathrm{~m}(K)\left|H_{K, \sigma}\right| \chi(x, \sigma, \xi)+\nu_{L} \mathrm{~m}(L)\left|H_{L, \sigma}\right| \chi(x, \sigma, \xi) \\
\leq & \sum_{K \in \mathcal{M}} \operatorname{diam}(K)\left|\mathbf{r}_{K}\right| \sum_{\sigma \in \mathcal{E}_{K}} \chi(x, \sigma, \xi)+\sum_{K \in \mathcal{M}} \sum_{\sigma \in \mathcal{E}_{K}} \nu_{K} \mathrm{~m}(K)\left|H_{K, \sigma}\right| \chi(x, \sigma, \xi) .
\end{aligned}
$$

But $\chi(x, \sigma, \xi) \neq 0$ only if $x$ lies in the set $\sigma-[0,1] \xi$, which measure is $\mathrm{m}(\sigma)\left|\xi \cdot \mathbf{n}_{\sigma}\right| \leq \mathrm{m}(\sigma)|\xi|\left(\mathbf{n}_{\sigma}\right.$ being a unit normal to $\sigma)$. Hence, integrating the preceding inequality on $x \in \omega$,

$$
\int_{\omega}|u(x+\xi)-u(x)| d x \leq \sum_{K \in \mathcal{M}} \operatorname{diam}(K)\left|\mathbf{r}_{K}\right| \sum_{\sigma \in \mathcal{E}_{K}} \mathrm{~m}(\sigma)|\xi|+\sum_{K \in \mathcal{M}} \sum_{\sigma \in \mathcal{E}_{K}} \nu_{K} \mathrm{~m}(K)\left|H_{K, \sigma}\right| \mathrm{m}(\sigma)|\xi|
$$

and we conclude using the fact that $\mathrm{m}(\sigma) \leq \omega_{d-1} \operatorname{diam}(K)^{d-1}$ whenever $\sigma \in \mathcal{E}_{K}$, that $\operatorname{Card}\left(\mathcal{E}_{K}\right) \leq$ $\operatorname{regul}(\mathcal{D}) \leq \theta$ and inequality $(2.1)$

Lemma 7.4 Let $\Omega$ be a convex polygonal bounded open subset of $\mathbb{R}^{d}$ and $T>0$. Let $\left(\mathcal{D}_{m}\right)_{m>1}$ be a sequence of admissible discretizations of $\Omega$ such that $\operatorname{size}\left(\mathcal{D}_{m}\right) \rightarrow 0$ as $m \rightarrow \infty$ and $\left(\operatorname{regul}\left(\mathcal{D}_{m}\right)\right)_{m \geq 1}$ is bounded. We also take, for all $m \geq 1, k_{m}>0$ such that $N_{k_{m}}=T / k_{m}$ is an integer and $k_{m} \rightarrow 0$ as $m \rightarrow \infty$, and $\nu_{m}=\left(\nu_{m, K}\right)_{K \in \mathcal{M}_{m}}$ a family of positive numbers.

For all $m \geq 1$ and all $n=1, \ldots, N_{k_{m}}$, we take $\left(u^{m, n}, \mathbf{r}^{m, n}\right)=\left(u_{K}^{m, n}, \mathbf{r}_{K}^{m, n}\right)_{K \in \mathcal{M}_{m}}$ and a family $H^{m, n}=$ $\left(H_{K, \sigma}^{m, n}\right)_{K \in \mathcal{M}_{m}, \sigma \in \mathcal{E}_{K}}$ such that $\left(u^{m, n}, \mathbf{r}^{m, n}, H^{m, n}\right) \in L_{\nu_{m}}\left(\mathcal{D}_{m}\right)$. We let $\left(u^{m}, \mathbf{r}^{m}\right)$ be the functions on $\left[0, T\left[\times \Omega\right.\right.$ equal to $\left(u_{K}^{m, n}, \mathbf{r}_{K}^{m, n}\right)$ on $\left[(n-1) k, n k\left[\times K\left(\right.\right.\right.$ for $n=1, \ldots, N_{k_{m}}$ and $\left.K \in \mathcal{M}_{m}\right)$.

Assume that, as $m \rightarrow \infty, u^{m} \rightarrow \bar{u}$ weakly in $L^{2}(] 0, T[\times \Omega), \mathbf{r}^{m} \rightarrow \overline{\mathbf{r}}$ weakly in $L^{2}(] 0, T[\times \Omega)^{d}$ and

$$
\sum_{n=1}^{N_{k_{m}}} k_{m} \sum_{K \in \mathcal{M}_{m}} \sum_{\sigma \in \mathcal{E}_{K}} \operatorname{diam}(K)^{d-1} \nu_{m, K} \mathrm{~m}(K)\left|H_{K, \sigma}^{m, n}\right| \rightarrow 0 .
$$

Then $\bar{u} \in L^{2}\left(0, T ; H^{1}(\Omega)\right)$ and $\nabla \bar{u}=\overline{\mathbf{r}}$.

\section{Proof of Lemma 7.4}

We first simplify the notations by dropping the index $m$; hence, we denote $\mathcal{D}=\mathcal{D}_{m}, k=k_{m}, u=u^{m}$, $\mathbf{r}=\mathbf{r}^{m}, H_{K, \sigma}^{n}=H_{K, \sigma}^{m, n}$, and we are interested in the convergence of quantities as $k \rightarrow 0$ and $\operatorname{size}(\mathcal{D}) \rightarrow 0$.

To prove the lemma, we just need to show that $\nabla \bar{u}=\overline{\mathbf{r}}$ in the sense of the distributions on $] 0, T[\times \Omega$. Let $\varphi \in C_{c}^{\infty}(] 0, T[\times \Omega)$ and $\mathbf{e} \in \mathbb{R}^{d}$; we multiply each equation (7.1) on $\left(u^{n}, \mathbf{r}^{n}, H^{n}\right)$ by $\int_{(n-1) k}^{n k} \int_{\sigma} \varphi \mathbf{e} \cdot \mathbf{n}_{K, \sigma} d \gamma$. We then sum on the edges and, using $\mathbf{n}_{K, \sigma}=-\mathbf{n}_{L, \sigma}$ if $\sigma=K \mid L \in \mathcal{E}_{\text {int }}$, we gather by control volumes. Thanks to the fact that $\int_{(n-1) k}^{n k} \int_{\sigma} \varphi \mathbf{e} \cdot \mathbf{n}_{K, \sigma} d \gamma=0$ if $\sigma \in \mathcal{E}_{\text {ext }}$, we can freely introduce the terms corresponding to boundary edges (which are otherwise not present). Finally summing on $n=1, \ldots, N_{k}$, we obtain

$$
\sum_{n=1}^{N_{k}} \sum_{K \in \mathcal{M}} \mathbf{r}_{K}^{n} \cdot \sum_{\sigma \in \mathcal{E}_{K}} \int_{(n-1) k}^{n k} \int_{\sigma} \varphi \mathbf{e} \cdot \mathbf{n}_{K, \sigma} d \gamma\left(\mathbf{x}_{\sigma}-\mathbf{x}_{K}\right)
$$




$$
\begin{aligned}
+\sum_{n=1}^{N_{k}} \sum_{K \in \mathcal{M}} \sum_{\sigma \in \mathcal{E}_{K}} \nu_{K} \mathrm{~m}(K) H_{K, \sigma}^{n} \int_{(n-1) k}^{n k} \int_{\sigma} \varphi \mathbf{e} \cdot \mathbf{n}_{K, \sigma} d \gamma & =-\sum_{n=1}^{N_{k}} \sum_{K \in \mathcal{M}} u_{K}^{n} \sum_{\sigma \in \mathcal{E}_{K}} \int_{(n-1) k}^{n k} \int_{\sigma} \varphi \mathbf{e} \cdot \mathbf{n}_{K, \sigma} d \gamma \\
& =-\sum_{n=1}^{N_{k}} \sum_{K \in \mathcal{M}} u_{K}^{n} \int_{(n-1) k}^{n k} \int_{K} \operatorname{div}(\varphi \mathbf{e}) \\
& =-\int_{0}^{T} \int_{\Omega} u \operatorname{div}(\varphi \mathbf{e}) .
\end{aligned}
$$

By convergence of $u$, this right-hand side tends, as $k \rightarrow 0$ and $\operatorname{size}(\mathcal{D}) \rightarrow 0$, to $-\int_{0}^{T} \int_{\Omega} \bar{u} \operatorname{div}(\varphi \mathbf{e})$. Let us denote $T_{11}$ and $T_{12}$ the two terms in the left-hand side of this equality.

We have, since $\varphi$ is bounded,

$$
\begin{aligned}
\left|T_{12}\right| & \leq\|\varphi\|_{\infty} \sum_{n=1}^{N_{k}} \sum_{K \in \mathcal{M}} \sum_{\sigma \in \mathcal{E}_{K}} \nu_{K} \mathrm{~m}(K)\left|H_{K, \sigma}^{n}\right| \mathrm{m}(\sigma) k \\
& \leq\|\varphi\|_{\infty} \omega_{d-1} \sum_{n=1}^{N_{k}} k \sum_{K \in \mathcal{M}} \sum_{\sigma \in \mathcal{E}_{K}} \nu_{K} \mathrm{~m}(K)\left|H_{K, \sigma}^{n}\right| \operatorname{diam}(K)^{d-1}
\end{aligned}
$$

and thus, by assumption, $T_{12} \rightarrow 0$ as $k \rightarrow 0$ and $\operatorname{size}(\mathcal{D}) \rightarrow 0$. We now compare $T_{11}$ with

$$
T_{13}=\sum_{n=1}^{N_{k}} \sum_{K \in \mathcal{M}} \mathbf{r}_{K}^{n} \cdot \int_{(n-1) k}^{n k} \sum_{\sigma \in \mathcal{E}_{K}} \mathrm{~m}(\sigma)\left(\frac{1}{\mathrm{~m}(K)} \int_{K} \varphi \mathbf{e}\right) \cdot \mathbf{n}_{K, \sigma}\left(\mathbf{x}_{\sigma}-\mathbf{x}_{K}\right) .
$$

Since $\varphi$ is regular, we have $C_{43}$ only depending on $\varphi$ such that

$$
\begin{aligned}
\left|T_{11}-T_{13}\right| & \leq C_{43} \operatorname{size}(\mathcal{D}) \sum_{n=1}^{N_{k}} k \sum_{K \in \mathcal{M}}\left|\mathbf{r}_{K}^{n}\right| \sum_{\sigma \in \mathcal{E}_{K}} \mathrm{~m}(\sigma) \operatorname{diam}(K) \\
& \leq C_{43} \operatorname{size}(\mathcal{D}) \omega_{d-1} \sum_{n=1}^{N_{k}} k \sum_{K \in \mathcal{M}}\left|\mathbf{r}_{K}^{n}\right| \operatorname{Card}\left(\mathcal{E}_{K}\right) \operatorname{diam}(K)^{d} .
\end{aligned}
$$

Using the fact that $\operatorname{regul}(\mathcal{D})$ stays bounded, we get $C_{44}$ not depending on $k$ nor $\mathcal{D}$ such that

$$
\left|T_{11}-T_{13}\right| \leq C_{44} \operatorname{size}(\mathcal{D}) \sum_{n=1}^{N_{k}} k \sum_{K \in \mathcal{M}} \mathrm{m}(K)\left|\mathbf{r}_{K}^{n}\right|=C_{44} \operatorname{size}(\mathcal{D})\|\mathbf{r}\|_{L^{1}(] 0, T[\times \Omega)^{d}} .
$$

Since $\mathbf{r}$ is bounded in $L^{2}(] 0, T[\times \Omega)^{d}$, this shows that $T_{11}-T_{13} \rightarrow 0$ as size $(\mathcal{D}) \rightarrow 0$. Using Lemma 7.1 with $\frac{1}{\mathrm{~m}(K)} \int_{K} \varphi(t, \cdot) \mathbf{e}$ instead of $\mathbf{e}$, we have

$$
T_{13}=\sum_{n=1}^{N_{k}} \sum_{K \in \mathcal{M}} \mathbf{r}_{K}^{n} \cdot \int_{(n-1) k}^{n k} \int_{K} \varphi \mathbf{e}=\int_{0}^{T} \int_{\Omega} \mathbf{r} \cdot \varphi \mathbf{e} \rightarrow \int_{0}^{T} \int_{\Omega} \overline{\mathbf{r}} \cdot \varphi \mathbf{e} \quad \text { as } k \rightarrow 0 \text { and } \operatorname{size}(\mathcal{D}) \rightarrow 0 .
$$

Hence, the limit of $(7.6)$ as $k \rightarrow 0$ and $\operatorname{size}(\mathcal{D}) \rightarrow 0$ gives $\int_{0}^{T} \int_{\Omega} \overline{\mathbf{r}} \cdot \varphi \mathbf{e}=-\int_{0}^{T} \int_{\Omega} \bar{u} \operatorname{div}(\varphi \mathbf{e})$, which concludes the proof.

\subsection{A compactness lemma}

Lemma 7.5 Let $\Omega$ be a bounded open subset of $\mathbb{R}^{d}, T>0$ and $A \subset L^{1}\left(0, T ; L_{\mathrm{loc}}^{1}(\Omega)\right)$. If $A$ is relatively compact in $L^{1}\left(0, T ;\left(C_{c}^{2}(\Omega)\right)^{\prime}\right)$ and if, for all $\omega$ relatively compact in $\Omega$,

$$
\sup _{u \in A}\|u(\cdot, \cdot+\xi)-u\|_{L^{1}(] 0, T[\times \omega)} \rightarrow 0 \quad \text { as }|\xi| \rightarrow 0,
$$

then $A$ is relatively compact in $L^{1}\left(0, T ; L_{\mathrm{loc}}^{1}(\Omega)\right)$. 


\section{Proof of Lemma 7.5}

The proof is inspired by classical proofs of Kolmogorov's or Aubin's compactness theorems. Let $\omega$ be relatively compact in $\Omega$ and take $\left(\rho_{\mu}\right)_{0<\mu<\operatorname{dist}\left(\omega, \mathbb{R}^{d} \backslash \Omega\right)}$ smoothing kernels on $\mathbb{R}^{d}$ such that $\operatorname{supp}\left(\rho_{\mu}\right)$ is included in the ball of center 0 and radius $\mu$. For $u \in A$, let $u_{\mu}=u * \rho_{\mu}$ (the convolution being only on the space variable), which is defined on $] 0, T[\times \omega$.

We first prove that, for all $\mu, A_{\mu}=\left\{u_{\mu}, u \in A\right\}$ is relatively compact in $L^{1}(] 0, T[\times \omega)$. Let $\left(u_{\mu}^{n}\right)_{n \geq 1}$ be a sequence in $A_{\mu}$. Since $\left(u^{n}\right)_{n>1}$ lies in $A$, it is relatively compact in $L^{1}\left(0, T ;\left(C_{c}^{2}(\Omega)\right)^{\prime}\right)$ and we can assume, up to a subsequence, that it converges in this space. We then have, for all $(t, x) \in] 0, T[\times \omega$, since $\operatorname{supp}\left(\rho_{\mu}(x-\cdot)\right) \subset \Omega$ by choice of $\mu$,

$\left|u_{\mu}^{n}(t, x)-u_{\mu}^{m}(t, x)\right|=\left|\int_{\Omega}\left(u^{n}(t, y)-u^{m}(t, y)\right) \rho_{\mu}(x-y) d x\right| \leq\left\|u^{n}(t, \cdot)-u^{m}(t, \cdot)\right\|_{\left(C_{c}^{2}(\Omega)\right)^{\prime}}\left\|\rho_{\mu}(x-\cdot)\right\|_{C_{c}^{2}(\Omega)}$.

Hence, integrating on $x \in \omega$ and $t \in] 0, T$, we find $C_{\mu}$ depending on $\mu$ but not on $n$ nor $m$ such that $\| u_{\mu}^{n}-$ $u_{\mu}^{m}\left\|_{L^{1}(] 0, T[\times \omega)} \leq C_{\mu}\right\| u^{n}-u^{m} \|_{L^{1}\left(0, T ;\left(C_{c}^{2}(\Omega)\right)^{\prime}\right)}$, which shows that $\left(u_{\mu}^{n}\right)_{n \geq 1}$ converges in $L^{1}(] 0, T[\times \omega)$ since $\left(u^{n}\right)_{n \geq 1}$ converges in $L^{1}\left(0, T ;\left(C_{c}^{2}(\Omega)\right)^{\prime}\right)$. Hence, for all $\left.\mu \in\right] 0, \operatorname{dist}\left(\omega, \mathbb{R}^{d} \backslash \Omega\right)\left[, A_{\mu}\right.$ is relatively compact in $L^{1}(] 0, T[\times \omega)$.

Let us now conclude the proof. It is sufficient to show that $\sup _{u \in A}\left\|u-u_{\mu}\right\|_{L^{1}(] 0, T[\times \omega)} \rightarrow 0$ as $\mu \rightarrow 0$. Indeed, once this is done, we have $A \subset A_{\mu}+B_{L^{1}(] 0, T[\times \omega)}(0, \delta(\mu))$ with $\delta(\mu) \rightarrow 0$ as $\mu \rightarrow 0$, which clearly shows, since $A_{\mu}$ is precompact in $L^{1}(] 0, T[\times \omega)$, that $A$ is also precompact (and thus relatively compact) in this space.

Let $u \in A, t \in] 0, T\left[\right.$ and $x \in \omega$; we have $\left|u(t, x)-u_{\mu}(t, x)\right| \leq \int_{B(0, \mu)}|u(t, x)-u(t, x-y)| \rho_{\mu}(y) d y$ and thus, integrating on $x \in \omega$ and $t \in] 0, T[$,

$$
\begin{aligned}
\left\|u-u_{\mu}\right\|_{L^{1}(] 0, T[\times \omega)} & \leq \int_{B(0, \mu)} \int_{0}^{T} \int_{\omega}|u(t, x)-u(t, x-y)| d t d x \rho_{\mu}(y) d y \\
& \leq \sup _{|y| \leq \mu} \int_{0}^{T} \int_{\omega}|u(t, x)-u(t, x-y)| d t d x,
\end{aligned}
$$

and the proof is concluded.

\subsection{A technical lemma}

Lemma 7.6 Let $\Omega$ be a convex polygonal bounded open subset of $\mathbb{R}^{d}, T>0$ and $A: \Omega \times \mathbb{R} \rightarrow M_{d}(\mathbb{R})$ a Caratheodory bounded matrix-valued function. Let $\left(\mathcal{D}_{m}\right)_{m \geq 1}$ be a sequence of admissible discretizations of $\Omega$ such that $\operatorname{size}\left(\mathcal{D}_{m}\right) \rightarrow 0$ as $m \rightarrow \infty$, and $k_{m}>0$ be such that $N_{k_{m}}=T / k_{m}$ is an integer and $k_{m} \rightarrow 0$ as $m \rightarrow \infty$.

Let $u^{m}=\left(u_{K}^{m, n}\right)_{n=1, \ldots, N_{k_{m}}}, K \in \mathcal{M}$ be a function on $] 0, T[\times \Omega$, constant on each $[(n-1) k, n k[\times K(n=$ $\left.1, \ldots, N_{k_{m}}, K \in \mathcal{M}_{m}\right)$. We assume that $u^{m} \rightarrow \bar{u}$ in $L^{1}\left(0, T ; L_{\text {loc }}^{1}(\Omega)\right)$ as $m \rightarrow \infty$. Let $\mathbf{Z}^{m} \in$ $L^{2}(] 0, T[\times \Omega)^{d}$ which converges to $\overline{\mathbf{Z}}$ in $L^{2}(] 0, T[\times \Omega)^{d}$ as $m \rightarrow \infty$. Define $A_{\mathcal{D}_{m}}: \Omega \times \mathbb{R} \rightarrow M_{d}(\mathbb{R})$ by $A_{\mathcal{D}_{m}}(x, s)=\frac{1}{\mathrm{~m}(K)} \int_{K} A(y, s) d y$ whenever $x$ belongs to $K \in \mathcal{M}_{m}$.

Then $A_{\mathcal{D}_{m}}\left(\cdot, u^{m}\right) \mathbf{Z}^{m} \rightarrow A(\cdot, \bar{u}) \overline{\mathbf{Z}}$ in $L^{2}(] 0, T[\times \Omega)^{d}$ as $m \rightarrow \infty$.

\section{Proof of Lemma 7.6}

To simplify the notations, we drop the index $m$ and we study the convergence as $k \rightarrow 0$ and $\operatorname{size}(\mathcal{D}) \rightarrow 0$. Let us first prove that, up to a subsequence, $A_{\mathcal{D}}(\cdot, u) \rightarrow A(\cdot, \bar{u})$ in $L^{1}(] 0, T[\times \Omega)^{d \times d}$. We have, since $u_{K}^{n}=u(t, y)$ if $(t, y) \in[(n-1) k, n k[\times K$,

$$
\int_{0}^{T} \int_{\Omega}\left|A_{\mathcal{D}}(x, u(t, x))-A(x, \bar{u}(t, x))\right| d t d x
$$




$$
\begin{aligned}
= & \sum_{n=1}^{N_{k}} \int_{(n-1) k}^{n k} \sum_{K \in \mathcal{M}} \int_{K}\left|\frac{1}{\mathrm{~m}(K)} \int_{K} A\left(y, u_{K}^{n}\right) d y-A(x, \bar{u}(t, x))\right| d t d x \\
\leq & \sum_{n=1}^{N_{k}} \int_{(n-1) k}^{n k} \sum_{K \in \mathcal{M}} \frac{1}{\mathrm{~m}(K)} \int_{K} \int_{K}|A(y, u(t, y))-A(x, \bar{u}(t, x))| d t d x d y \\
\leq & \sum_{n=1}^{N_{k}} \int_{(n-1) k}^{n k} \sum_{K \in \mathcal{M}} \frac{1}{\mathrm{~m}(K)} \int_{K} \int_{K}|A(y, u(t, y))-A(y, \bar{u}(t, y))| d t d x d y \\
& +\sum_{n=1}^{N_{k}} \int_{(n-1) k}^{n k} \sum_{K \in \mathcal{M}} \frac{1}{\mathrm{~m}(K)} \int_{K} \int_{K}|A(y, \bar{u}(t, y))-A(x, \bar{u}(t, x))| d t d x d y \\
\leq & \int_{0}^{T} \int_{\Omega}|A(y, u(t, y))-A(y, \bar{u}(t, y))| d t d y \\
& +\sum_{n=1}^{N_{k}} \int_{(n-1) k}^{n k} \sum_{K \in \mathcal{M}} \frac{1}{\mathrm{~m}(K)} \int_{K} \int_{K}|A(y, \bar{u}(t, y))-A(x, \bar{u}(t, x))| d t d x d y .
\end{aligned}
$$

Up to a subsequence, we can assume that $u \rightarrow \bar{u}$ a.e. on $] 0, T[\times \Omega$. Since $A$ is bounded on $\Omega \times \mathbb{R}$ and $A(y, \cdot)$ is continuous, the dominated convergence theorem shows that $(7.7)$ tends to 0 as $k \rightarrow 0$ and $\operatorname{size}(\mathcal{D}) \rightarrow 0$. The function $A(\cdot, \bar{u})$ is integrable on $] 0, T\left[\times \Omega\right.$; for all $\varepsilon>0$, we can therefore find $\mathcal{F} \in C_{c}^{1}(] 0, T[\times \Omega) d \times d$ such that $\|\mathcal{F}-A(\cdot, \bar{u})\|_{L^{1}(] 0, T[\times \Omega)^{d \times d}} \leq \varepsilon$ and we then have

$$
\begin{aligned}
(7.8) \leq & \sum_{n=1}^{N_{k}} \int_{(n-1) k}^{n k} \sum_{K \in \mathcal{M}} \frac{1}{\mathrm{~m}(K)} \int_{K} \int_{K}|A(y, \bar{u}(t, y))-\mathcal{F}(t, y)| d t d x d y \\
& +\sum_{n=1}^{N_{k}} \int_{(n-1) k}^{n k} \sum_{K \in \mathcal{M}} \frac{1}{\mathrm{~m}(K)} \int_{K} \int_{K}|\mathcal{F}(t, y)-\mathcal{F}(t, x)| d t d x d y \\
& +\sum_{n=1}^{N_{k}} \int_{(n-1) k}^{n k} \sum_{K \in \mathcal{M}} \frac{1}{\mathrm{~m}(K)} \int_{K} \int_{K}|\mathcal{F}(t, x)-A(x, \bar{u}(t, x))| d t d x d y \\
\leq & \|A(\cdot, \bar{u})-\mathcal{F}\|_{L^{1}(] 0, T[\times \Omega) d \times d}+C_{45} \operatorname{size}(\mathcal{D})+\|\mathcal{F}-A(\cdot, \bar{u})\|_{L^{1}(] 0, T[\times \Omega)^{d \times d}} \\
\leq & 2 \varepsilon+C_{45} \operatorname{size}(\mathcal{D})
\end{aligned}
$$

where $C_{45}$ only depends on $\mathcal{F}, T$ and $\Omega$. Hence, (7.8) tends to 0 as $\operatorname{size}(\mathcal{D}) \rightarrow 0$ and we deduce that $A_{\mathcal{D}}(\cdot, u) \rightarrow A(\cdot, \bar{u})$ in $L^{1}(] 0, T[\times \Omega)^{d \times d}$.

Up to a subsequence, we can thus assume that $A_{\mathcal{D}}(\cdot, u) \rightarrow A(\cdot, \bar{u})$ a.e. on $] 0, T[\times \Omega$. By strong convergence of $\mathbf{Z}$ and since $A$ is bounded, it is then easy to deduce that $A_{\mathcal{D}}(\cdot, u) \mathbf{Z} \rightarrow A(\cdot, \bar{u}) \overline{\mathbf{Z}}$ in $L^{2}(] 0, T[\times \Omega)^{d}$. This has only been proved up to a subsequence, but since the limit is unique, this proves that the whole sequence converges.

\section{References}

[1] Amirat Y., Ziani A., Asymptotic behavior of the solutions of an elliptic-parabolic system arising in flow in porous media, Z. Anal. Anwendungen, 23 (2004) 2, pp. 335-351.

[2] Bear J., Dynamics of fluids in porous media, New York: American Elsevier, 1972.

[3] Chen Z., Ewing R., Mathematical analysis for reservoir models, SIAM J. Math. Anal., 30 (1999) 2, pp. 431-453. 
[4] Douglas J., Numerical methods for the flow of miscible fluids in porous media, Edt R.W. Lewis et al, New York: John Wiley, 1984.

[5] Douglas J., Ewing R. E., Wheeler M. F., The approximation of the pressure by a mixed method in the simulation of miscible displacement. RAIRO Anal. Numr., 17 (1983), no. 1, pp. 17-33.

[6] Douglas J., Ewing R. E., Wheeler M. F., A time-discretization procedure for a mixed finite element approximation of miscible displacement in porous media, RAIRO Anal. Num., 17 (1983), pp. 249-265.

[7] Droniou J., Intégration et Espaces de Sobolev à valeurs vectorielles, available at http://www-gm3.univ-mrs.fr/polys/

[8] Droniou J., Finite volume schemes for fully non-linear elliptic equations in divergence form, submitted. Available at http://hal.ccsd.cnrs.fr/ccsd-00009614.

[9] Droniou J., Eymard R., A mixed finite volume scheme for anisotropic diffusion problems on any grid, submitted. Available at http://hal.ccsd.cnrs.fr/ccsd-00005565.

[10] Ewing R., Russel T., Wheeler M., Simulation of miscible displacement using mixed methods and a modified method of characteristics, SPE 12241, (1983), pp. 71-81.

[11] Ewing R. E., Russell T. F., Efficient time-stepping methods for miscible displacement problems in porous media. SIAM J. Numer. Anal., 19 (1982), no. 1, pp. 1-67.

[12] Ewing R. E., Russell T. F., Wheeler M. F., Convergence analysis of an approximation of miscible displacement in porous media by mixed finite elements and a modified method of characteristics. Comput. Methods Appl. Mech. Engrg., 47 (1984), no. 1-2, pp. 73-92.

[13] Eymard R., Gallouët T., Herbin R., Finite Volume Methods, Handbook of Numerical Analysis, Vol. VII, pp. 713-1020. Edited by P.G. Ciarlet and J.L. Lions (North Holland).

[14] FAILlE I, A control volume method to solve an elliptic equation on a two-dimensional irregular mesh, Comput. Methods Appl. Mech. Eng, 100 (1992), pp. 275-290.

[15] FENG X., On existence and uniqueness results for a coupled system modeling miscible displacement in porous media, J. Math. Anal. Appl. 194 (1995) 3, pp. 883-910.

[16] Grisvard P., Elliptic Problems in Nonsmooth Domains, Pitman 1985.

[17] Lin P., YAng D., An iterative perturbation method for the pressure equation in the simulation of miscible displacement in porous media. SIAM J. Sci. Comput., 19 (1998), no. 3, pp. 893-911.

[18] Roberts J. E., Thomas, J.-M., Mixed and hybrid methods, Handbook of numerical analysis, Vol. II, pp. 523-639, North-Holland, Amsterdam, 1991.

[19] Russell T.F., Finite elements with characteritics for two-component incompressible miscible displacement, SPE 10500, Proc. 6th SPE symposium on Reservoir Simulation, New Orleans (1982), pp.123-135.

[20] Wang H., Liang D., Ewing R. E., Lyons S. L., QIn G., An approximation to miscible fluid flows in porous media with point sources and sinks by an Eulerian-Lagrangian localized adjoint method and mixed finite element methods, SIAM J. Sci. Comput., 22 (2000) 2, pp. 561-581 (electronic).

[21] Wang H., Liang D., Ewing R. E., Lyons S. L., Qin G., An improved numerical simulator for different types of flows in porous media, Numer. Methods Partial Differential Equations, 19 (2003) 3, pp. 343-362. 